Florida International University

FIU Digital Commons

FIU Electronic Theses and Dissertations

University Graduate School

$11-12-2009$

\title{
Orthogonal Frequency Division Multiplexing Based Air Interfaces and Multiple Input Multiple Output Techniques in Cooperative Satellite Communications for 4th Generation Mobile Systems
}

Yuri Labrador

Florida International University, ylabr001@fiu.edu

DOI: $10.25148 /$ etd.FI09120831

Follow this and additional works at: https://digitalcommons.fiu.edu/etd

\section{Recommended Citation}

Labrador, Yuri, "Orthogonal Frequency Division Multiplexing Based Air Interfaces and Multiple Input Multiple Output Techniques in Cooperative Satellite Communications for 4th Generation Mobile Systems" (2009). FIU Electronic Theses and Dissertations. 107.

https://digitalcommons.fiu.edu/etd/107 


\section{FLORIDA INTERNATIONAL UNIVERSITY}

Miami, Florida

ORTHOGONAL FREQUENCY DIVISION MULTIPLEXING BASED AIR INTERFACES AND MULTIPLE INPUT MULTIPLE OUTPUT TECHNIQUES IN COOPERATIVE SATELLITE COMMUNICATIONS FOR $4^{\text {th }}$ GENERATION MOBILE SYSTEMS

A dissertation submitted in partial fulfillment of the requirements for the degree of DOCTOR OF PHILOSOPHY

in ELECTRICAL ENGINEERING by Yuri Labrador 
To: Dean Amir Mirmiran

College of Engineering and Computing

This dissertation, written by Yuri Labrador, and entitled Orthogonal Frequency Division Multiplexing Based Air Interfaces and Multiple Input Multiple Output Techniques in Cooperative Satellite Communications for $4^{\text {th }}$ Generation Mobile Systems, having been approved in respect to style and intellectual content, is referred to you for judgment.

We have read this dissertation and recommend that it be approved.

Nikolaos Tsoukias

Deng Pan

Stavros V. Georgakopoulos

Kia Makki

Niki Pissinou, Major Professor

Date of Defense: November 12, 2009

The dissertation of Yuri Labrador is approved.

\author{
Dean Amir Mirmiran \\ College of Engineering and Computing \\ Dean George Walker \\ University Graduate School
}

Florida International University, 2009 
(C) Copyright 2009 by Yuri Labrador

All rights reserved. 


\section{DEDICATION}

I dedicate this dissertation to my parents Flavio and Clara, my wife Nersy and my daughter Clarice for their unconditional love, encouragement and support. 


\section{ACKNOWLEDGMENTS}

I would like to thank Dr. Niki Pissinou and Dr. Kia Makki for being so excellent professors and for their insights and guidance through all my years at FIU. Also to the members of my dissertation committee: Dr. Stavros V. Georgakopoulos, Dr. Deng Pan and Dr. Nikolaos Tsoukias for their time reading my dissertation and their participation in the committee.

I sincerely thank all the members of the FIU Engineering Center for their work and dedication. 


\begin{abstract}
OF THE DISSERTATION
ORTHOGONAL FREQUENCY DIVISION MULTIPLEXING BASED AIR

INTERFACES AND MULTIPLE INPUT MULTIPLE OUTPUT TECHNIQUES IN

COOPERATIVE SATELLITE COMMUNICATIONS FOR $4^{\mathrm{TH}}$ GENERATION

MOBILE SYSTEMS
\end{abstract}

by

Yuri Labrador

Florida International University, 2009

Miami, Florida

\title{
Professor Niki Pissinou, Major Professor
}

Recently, wireless network technology has grown at such a pace that scientific research has become a practical reality in a very short time span. Mobile wireless communications have witnessed the adoption of several generations, each of them complementing and improving the former. One mobile system that features high data rates and open network architecture is $4 \mathrm{G}$. Currently, the research community and industry, in the field of wireless networks, are working on possible choices for solutions in the $4 \mathrm{G}$ system. $4 \mathrm{G}$ is a collection of technologies and standards that will allow a range of ubiquitous computing and wireless communication architectures. The researcher considers one of the most important characteristics of future $4 \mathrm{G}$ mobile systems the ability to guarantee reliable communications from $100 \mathrm{Mbps}$, in high mobility links, to as high as 1 Gbps for low mobility users, in addition to high efficiency in the spectrum usage. 
On mobile wireless communications networks, one important factor is the coverage of large geographical areas. In 4G systems, a hybrid satellite/terrestrial network is crucial to providing users with coverage wherever needed. Subscribers thus require a reliable satellite link to access their services when they are in remote locations, where a terrestrial infrastructure is unavailable. Thus, they must rely upon satellite coverage.

Good modulation and access technique are also required in order to transmit high data rates over satellite links to mobile users. This technique must adapt to the characteristics of the satellite channel and also be efficient in the use of allocated bandwidth. Satellite links are fading channels, when used by mobile users. Some measures designed to approach these fading environments make use of: (1) spatial diversity (two receive antenna configuration); (2) time diversity (channel interleaver/ spreading techniques); and (3) upper layer FEC.

The author proposes the use of OFDM (Orthogonal Frequency Multiple Access) for the satellite link by increasing the time diversity. This technique will allow for an increase of the data rate, as primarily required by multimedia applications, and will also optimally use the available bandwidth. In addition, this dissertation approaches the use of Cooperative Satellite Communications for hybrid satellite/terrestrial networks. By using this technique, the satellite coverage can be extended to areas where there is no direct link to the satellite. For this purpose, a good channel model is necessary. 


\section{TABLE OF CONTENTS}

CHAPTER

PAGE

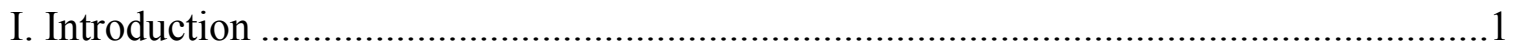

1.1 Background and Motivation .............................................................................

1.1.1 Orthogonal Frequency Multiple Access (OFDM) …………................................

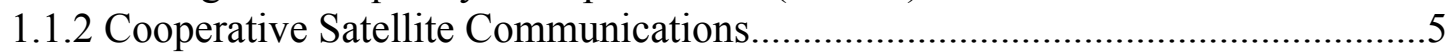

1.1.3 Characteristics of the Satellite Channel Model.....................................................6

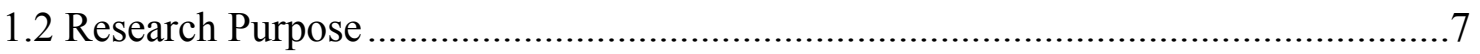

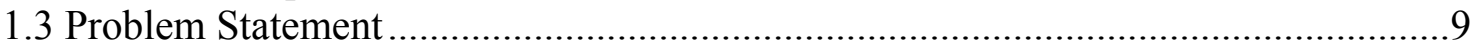

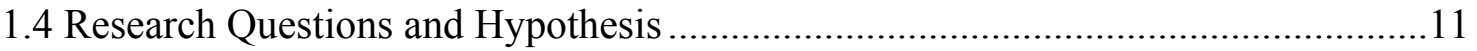

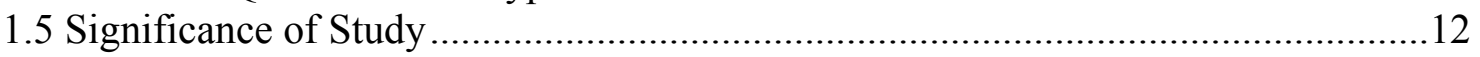

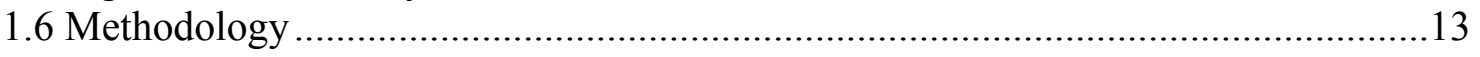

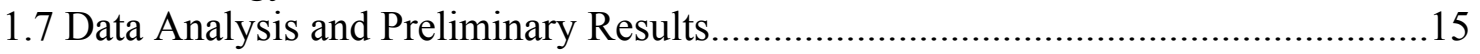

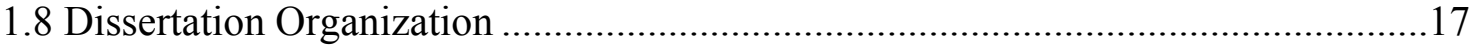

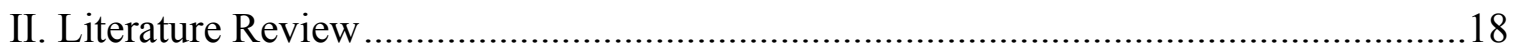

2.1 OFDM

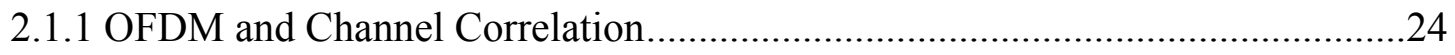

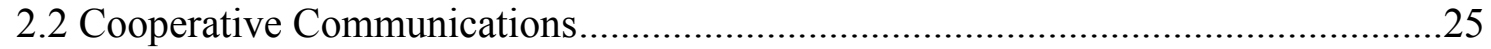

2.3 Challenges in Migration to 4G Mobile Systems ..................................................29

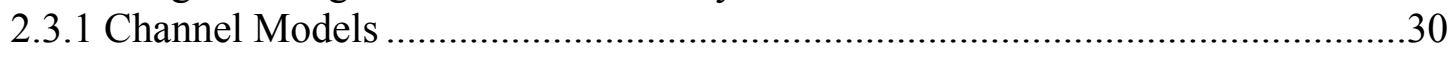

2.3.2 Channel Coding …………………………….............................................

III. Hybrid Satellite-Terrestrial Channel Model …………...............................................33

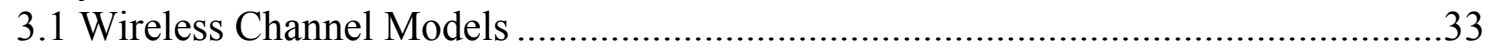

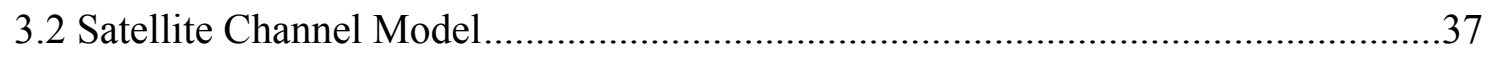

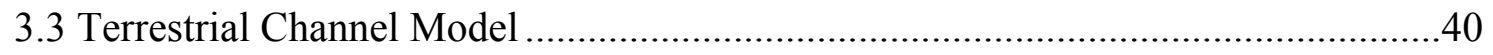

3.4 Hybrid Satellite-Terrestrial Channel Model ………….............................................43

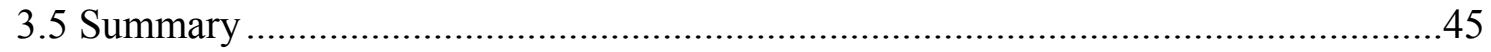

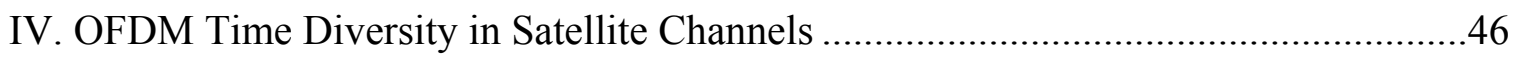

4.1 Discrete Time Signals and DFT ...........................................................................

4.2 OFDM

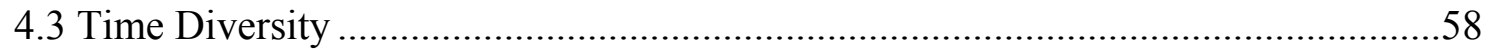

4.4 Non-linear Distortions in Satellite Channels .........................................................64

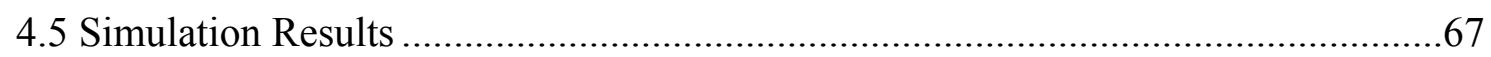

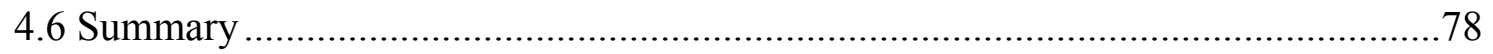

V. OFDM MIMO Space Diversity in Terrestrial Channels..............................................79

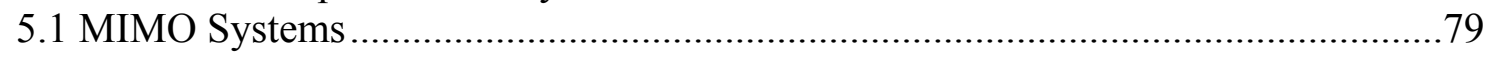

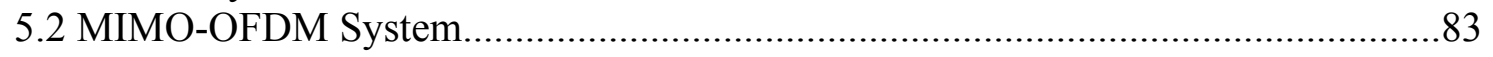

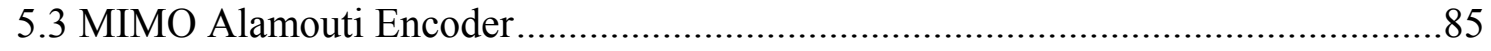




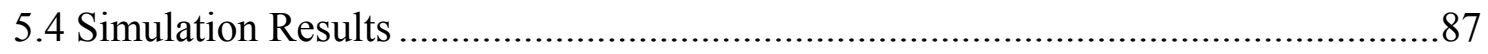

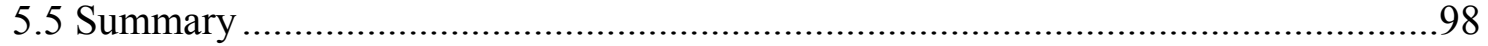

VI. An Approach to Cooperative Satellite Communications in 4G Mobile Systems......100

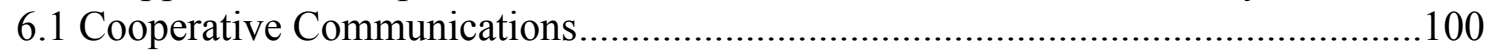

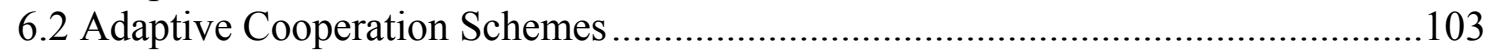

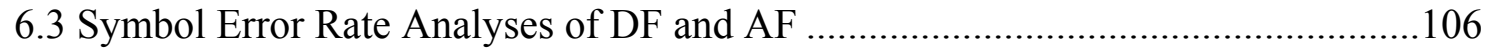

6.4 Analysis of Power Distribution in DF Schemes ................................................ 110

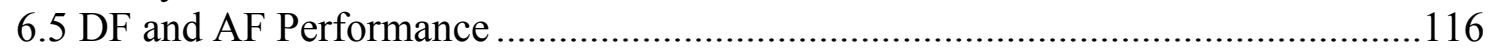

6.6 Selective and Incremental Relaying in Satellite/Terrestrial Cooperation ...............118

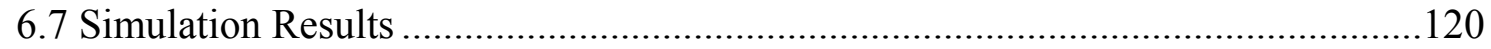

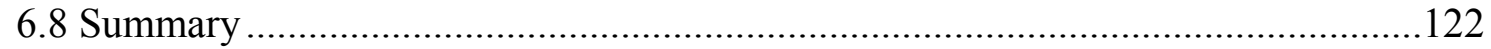

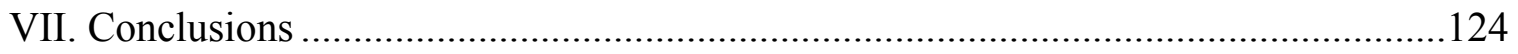

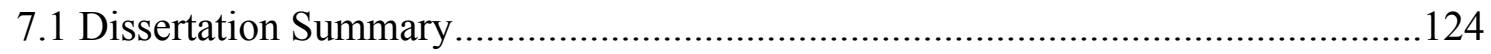

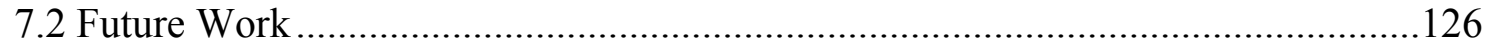

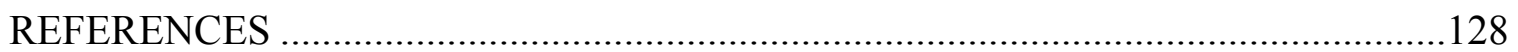

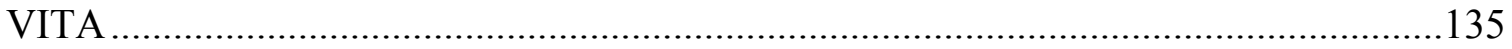




\section{LIST OF TABLES}

TABLE

PAGE

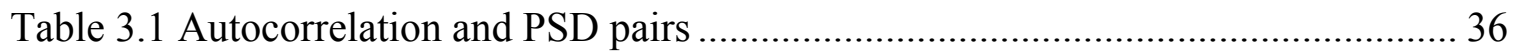

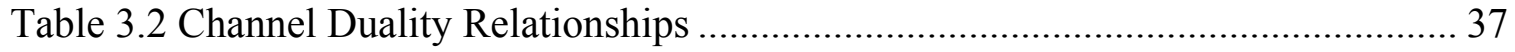

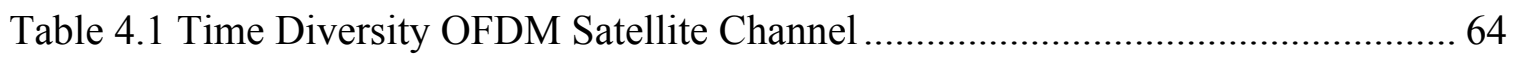

Table 4.2 Modulation Error Rate, OFDM Satellite Channel $\frac{E_{b}}{N o}=25 d B$...................... 74

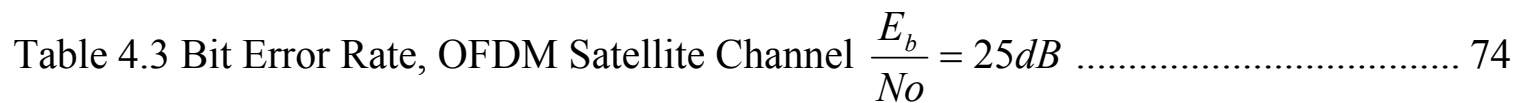

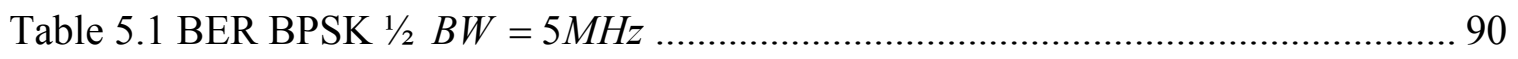

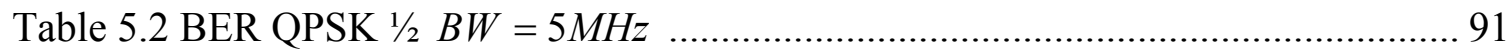

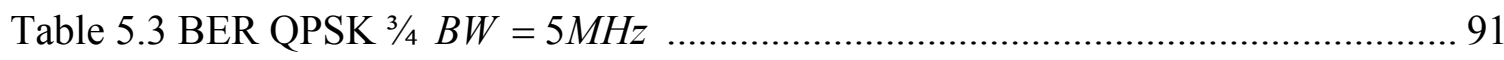

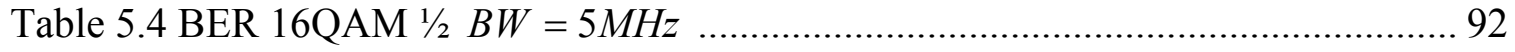

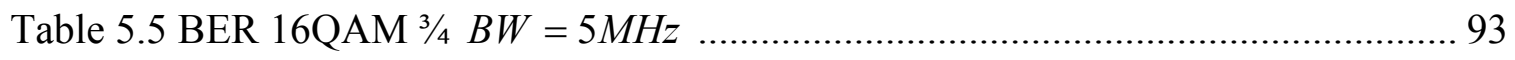

Table 7.1 Performance of OFDM Time Diversity in Satellite Channels...................... 125 


\section{LIST OF FIGURES}

FIGURE

PAGE

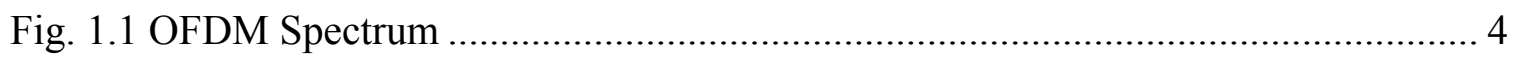

Fig. 1.2 64QAM Constellation and OFDM Spectrum ................................................ 7

Fig. 1.3 OFDM and Cooperative Satellite Communications ......................................... 8

Fig. 1.4 Decode and Forward will adapt to Bandwidth Allocations............................... 13

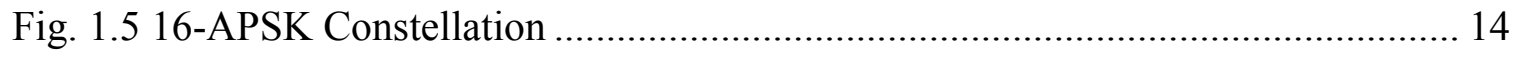

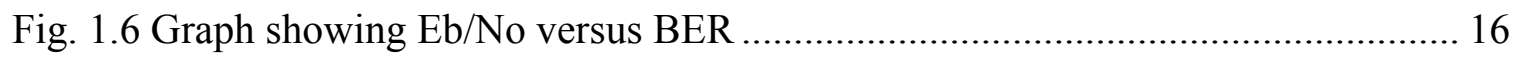

Fig. 1.7 Eb/No versus BER (no FEC) with PSK and QAM Modulation........................ 16

Fig. 2.1 Eb/No versus FER for Proposed Synchronization and Channel Estimation ....... 22

Fig. 2.2 SNR versus SER High Rate Spaced-Frequency Codes with Multiple CFO ....... 26

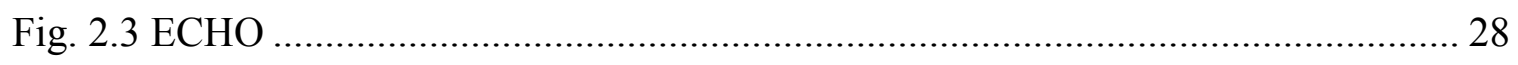

Fig. 3.1 Different Paths in a Wireless Link ............................................................ 33

Fig. 3.2 A Channel Model Using a Linear Filter ...................................................... 34

Fig. 3.3 Doppler Shift in a Single Frequency ......................................................... 35

Fig. 3.4 Probability Density Functions for Non-shadowing State ................................ 39

Fig. 3.5 Terrestrial Rayleigh Model................................................................... 40

Fig. 3.6 Hybrid Channel Model Architecture ...................................................... 44

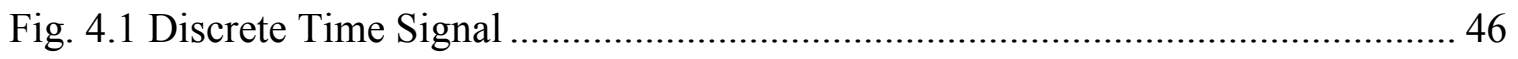

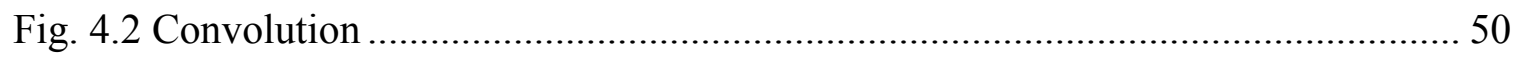

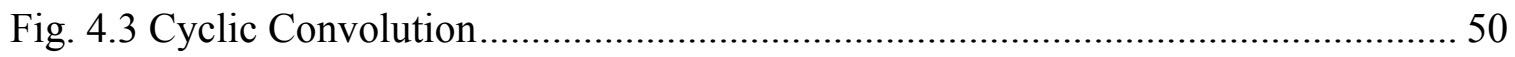

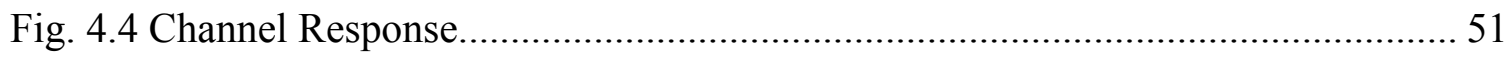

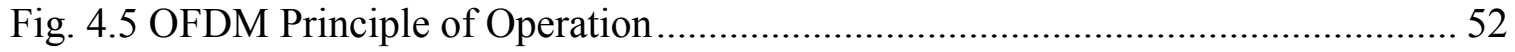




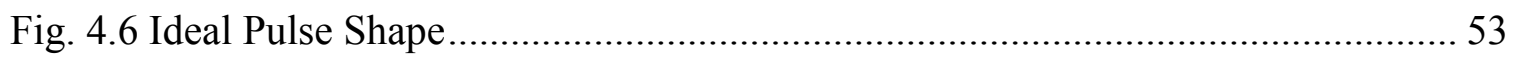

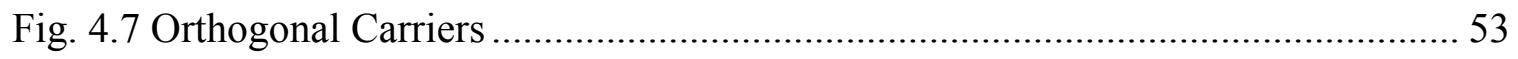

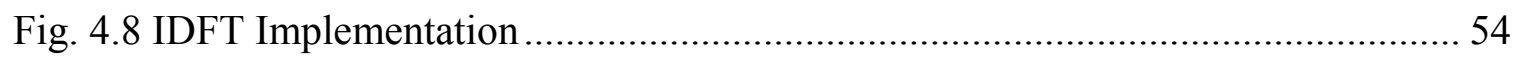

Fig. 4.9 OFDM Blocks at Transmitter ......................................................................... 54

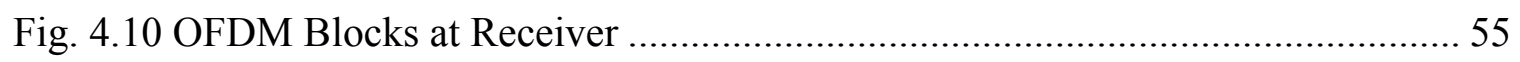

Fig. 4.11 OFDM Blocks with Extra Zeros added ....................................................... 55

Fig. 4.12 OFDM Received Blocks with No Overlapping.............................................. 56

Fig. 4.13 Received Signal as the Result of Cyclic Convolution...................................... 56

Fig. 4.14 OFDM Transmitter and Receiver ............................................................... 57

Fig. 4.15 Power Allocation for Different Sub-carriers ……………………………....... 58

Fig. 4.16 Transmitter Implementation Using Time Diversity ......................................... 61

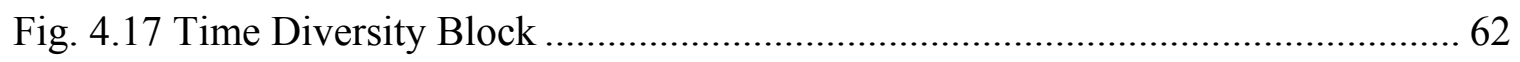

Fig. 4.18 Retransmission of Versions $($ ver 1, ver $2, \ldots .$, ver $N)$.......................................... 63

Fig. 4.19 Block Diagram of OFDM Satellite Link …………………........................... 67

Fig. 4.20 QPSK OFDM Saturation Level $=0$ db Relative to AM Average ..................... 68

Fig. 4.21 HPA Response for QPSK with Saturation Level of $0 \mathrm{~dB}$................................. 69

Fig. 4.22 OFDM QPSK Constellation Saturation Level 0 dB, Path Loss $=180 \mathrm{~dB}$......... 69

Fig. 4.23 QPSK OFDM Saturation Level 5 dB Relative to AM Average........................ 70

Fig. 4.24 HPA Response for QPSK with Saturation Level of $5 \mathrm{~dB}$............................... 70

Fig. 4.25 QPSK OFDM Constellation Saturation Level 5 dB, Path Loss $=180 \mathrm{~dB}$........ 71

Fig. 4.26 16QAM OFDM Saturation Level 0 dB Relative to AM Average...................... 72

Fig. 4.27 16QAM OFDM Constellation Saturation Level 0 dB, $\mathrm{K}=2$............................ 72

Fig. 4.28 16QAM OFDM Saturation Level 5 dB Relative to AM Average...................... 73 


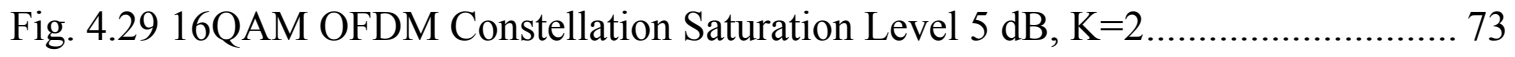

Fig. 4.30 BER Values QPSK OFDM Satellite Channel ........................................... 76

Fig. 4.31 BER Values 16QAM OFDM Satellite Channel .......................................... 76

Fig. 4.32 BER Values QPSK OFDM, Using Turbo Code......................................... 77

Fig. 4.33 BER Values 16QAM OFDM, Using Turbo Code........................................ 77

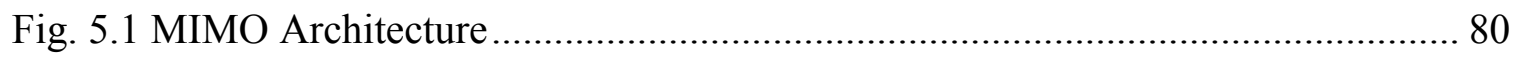

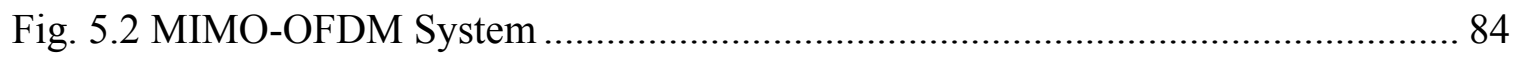

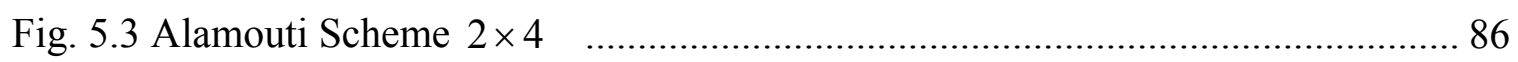

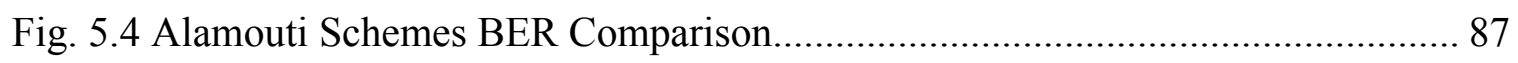

Fig. 5.5 MIMO OFDM Simulation Block Diagram …............................................. 87

Fig. 5.6 $\frac{E_{b}}{N_{0}}$ versus BER Results for BPSK, QPSK and 16QAM ................................. 89

Fig. 5.7 OFDM 16QAM 3/4 Transmitted Spectrum Antenna 1 ................................... 95

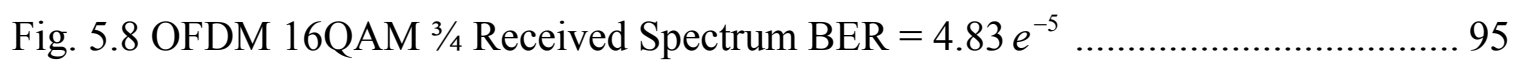

Fig. 5.9 16QAM 3/4 Transmitted (Blue) and Received (Red) Constellations BER =

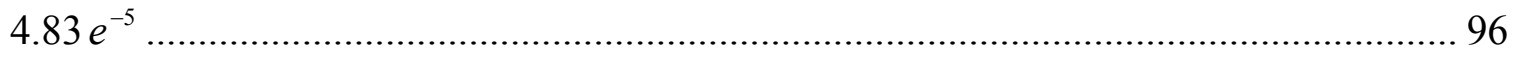

Fig. 5.10 QPSK 3/4 Transmitted and Received Constellations BER $=7.48 e^{-5} \ldots \ldots \ldots \ldots . . .96$

Fig. 5.11 QPSK 3/4 Transmitted and Received Eye Diagrams BER $=7.48 e^{-5} \ldots \ldots \ldots \ldots \ldots . . .97$

Fig. 5.12 16QAM 1/2 Transmitted and Received Eye Diagrams BER $=3.94 e^{-6} \ldots \ldots \ldots \ldots . .98$

Fig. 6.1 Cooperative Satellite Communications Showing Phase I and Phase II............ 101

Fig. 6.2 Phase II Occurs Only If the Destination Node Asks the Relay Node to Forward

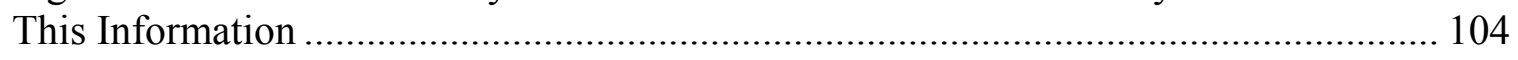

Fig. 6.3 Performance of Relaying Schemes versus SNR [dB] ................................. 105

Fig. 6.4 Performance of Relaying Schemes versus Rate [bps] ................................ 106 
Fig. 6.5 DF Cooperative Communications System with QPSK ................................ 110

Fig. 6.6 AF Cooperative Communications System with QPSK .................................. 110

Fig. 6.7 Case 3 - No Source-Destination Link ....................................................... 115

Fig. 6.8 Incremental and Selective Relaying .................................................... 119

Fig. 6.9 Both SNRs (at Destination and Relay) Are Low ......................................... 120

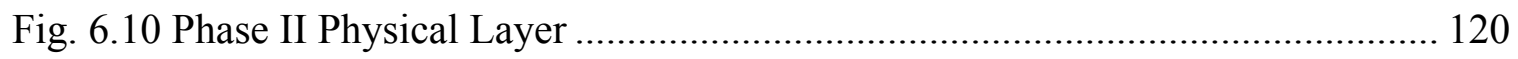

Fig. 6.11 OFDM Spectrum and Eye Diagram in Phase II Eb/No = $10 \mathrm{~dB} \ldots \ldots \ldots \ldots \ldots \ldots . . . . . .121$

Fig. 6.12 Eb/No versus SER OFDM 8PSK/16QAM Phase II................................... 122 


\section{Chapter I. Introduction}

\subsection{Background and Motivation}

Recently, wireless network technology has grown at such a pace that scientific research has become a practical reality in a very short time span. Mobile wireless communications have witnessed the adoption of several generations, each of them complementing and improving the former. First generation $(1 \mathrm{G})$ radios were based on analog signals and analog modulation techniques, primarily to transmit voice. Examples of these include AMPS (Advanced Mobile Phone Service) and TACS (Total Access Communication Systems), both of which were implemented during the 1980s. The second generation $(2 \mathrm{G})$ of wireless mobile systems was developed in the 1990 s and included such digital communications techniques as GSM (Global System for Mobile Communications), CDMA (Code Division Multiple Access), and PDC (Personal Digital cellular). Of those mentioned above, the one most widely used in 2G systems was GSM. The third generation $(3 \mathrm{G})$ of mobile systems allowed subscribers to access higher data rates than those allowed by $2 \mathrm{G}$. This evolution from $2 \mathrm{G}$ to $3 \mathrm{G}$ engendered many wireless systems, including Bluetooth, WLAN and HiperLAN. These systems differ from one another with respect to the users they are designed to serve, as well as the data rates and services they provide. $3 \mathrm{G}$ uses WCDMA (Wideband CDMA), time-division synchronous CDMA and cdma2000. One problem with $3 \mathrm{G}$ arises when high data rates are required (100 Mbps), using CDMA, because excessive interference among services occurs. Another problem with $3 \mathrm{G}$ is that it is very difficult to guarantee a full range of variable rate services, when each one requires a different grade of Quality of Service (QoS). This is due in part to the limitations in the backbone network by the air interference standards. 
One mobile system that features high data rates and open network architecture is 4G. Currently, the research community and industry, in the field of wireless networks, are working on possible choices for solutions in the $4 \mathrm{G}$ system. $4 \mathrm{G}$ is a collection of technologies and standards that will allow a range of ubiquitous computing and wireless communication architectures. From the user's point of view, some important characteristics of the 4G mobile systems are as follows [1]: (1) high availability anytime, anywhere and with any technology; (2) multimedia services at low costs; (3) personalization; and (4) integrated services.

The researcher considers one of the most important characteristics of future $4 \mathrm{G}$ mobile systems the ability to guarantee reliable communications from $100 \mathrm{Mbps}$, in high mobility links, to as high as 1 Gbps for low mobility users, in addition to high efficiency in the spectrum usage. $4 \mathrm{G}$ networks are IP-based, heterogeneous networks that allow users to employ any system, anytime, not only for telecommunication services, but also for data transfer and multimedia. By the time $4 \mathrm{G}$ functions seamlessly, it is expected that users in different locations and of distinct economic classes and occupations will all be able to use it. They will thus receive many different services from only one service provider. Researchers are faced with a number of challenges, however, that must be first addressed in order to allow this high level of integration.

On mobile wireless communications networks, one important factor is the coverage of large geographical areas. In 4G systems, a hybrid satellite/terrestrial network is crucial to providing users with coverage wherever needed. Subscribers thus require a reliable satellite link to access their services when they are in remote locations, where a terrestrial infrastructure is unavailable. Thus, they must rely upon satellite coverage. 
Good modulation [49] and access technique are also required in order to transmit high data rates over satellite links to mobile users. This technique must adapt to the characteristics of the satellite channel [20] and also be efficient in the use of allocated bandwidth. Satellite links are fading channels, when used by mobile users. Some measures designed to approach these fading environments make use of: (1) spatial diversity (two receive antenna configuration); (2) time diversity (channel interleaver/ spreading techniques); and (3) upper layer FEC.

The author proposes the use of OFDM (Orthogonal Frequency Multiple Access) for the satellite link by increasing the time diversity. This technique will allow for an increase of the data rate, as primarily required by multimedia applications [19], and will also optimally use the available bandwidth. In addition, this paper approaches the use of Cooperative Satellite Communications for hybrid satellite/terrestrial networks. By using this technique, the satellite coverage can be extended to areas where there is no direct link to the satellite. For this purpose, a good channel model is necessary.

\subsubsection{Orthogonal Frequency Multiple Access (OFDM)}

OFDM has become increasingly popular for the transmission of signals over wireless channels. It functions by dividing the available bandwidth into sub-channels, so the signal to be transmitted is the sum of all sub-carriers (see Figure 1.1). Although the frequency components of the signal overlap with one another, the sub-carriers are selected orthogonally so the available bandwidth is used very efficiently. By using the appropriate bit loading methods on the dynamic property of the channel previously estimated, the OFDM adapts its transmitted signal to the channel properties. 
Some advantages of OFDM include the following: (1) outstanding spectral efficiency; (2) implementation based on FFT (Fast Fourier Transform), which makes it very simple; (3) low receiver complexity; (4) high data rate transmission over Multipath Fading Channels; (5) link adaptation; and (6) very strong Inter Symbol Interference (ISI) performance. OFDM has been used in many wireless standards, e.g., Digital Audio Broadcasting (DAB), terrestrial Digital Video Broadcasting (DVB-T), and the IEEE 802.11a wireless LAN.

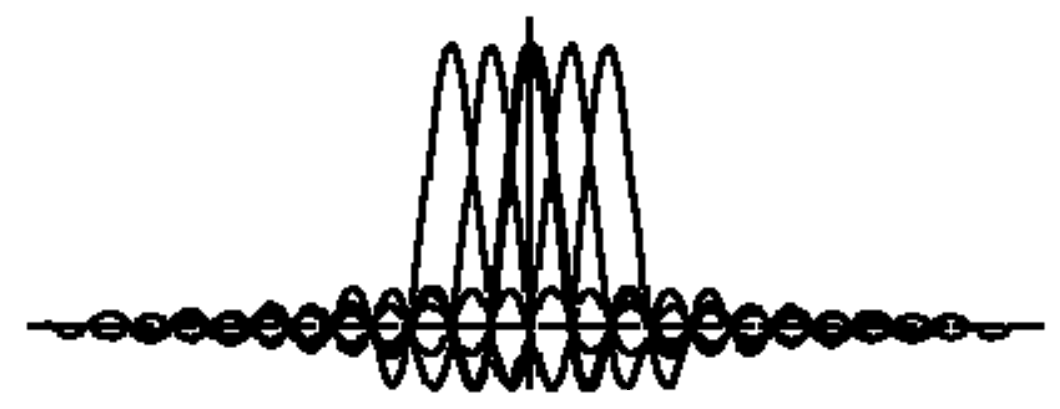

Fig. 1.1 OFDM Spectrum

MIMO (Multiple Inputs Multiple Outputs) is a technique for space diversity that uses many transmission and reception antennas. MIMO can be implemented in various ways, either to gain diversity, to combat signal fading, or to obtain capacity gain. MIMO enables the exploration of a new dimension and offers the benefits of diversity. It has been adopted by several standards, along with OFDM. A MIMO-OFDM combination can be very attractive and is currently the most promising broadband wireless technique. MIMO-OFDM decreases receiver complexity, while MIMO increases capacity. In high data rate links, the multipath characteristics of the channel make MIMO frequencyselective. OFDM converts this frequency selective channel into a group of parallel frequency flat channels. 


\subsubsection{Cooperative Satellite Communications}

Satellite coverage is essential to future $4 \mathrm{G}$ mobile systems, but unfortunately, there are certain areas unreachable by satellites. These areas can be found indoors and in tunnels, so a way to relay signals to those areas must be developed. Cooperative Communications is more expansive than signal amplification, so it has been studied by researchers as a way to extend coverage. Cooperative Communications [2] enables users to cooperate in order to obtain and provide higher data rates and service quality, by employing a source node that multicasts the signal to a group of relays nodes. These relay nodes process and retransmit a version of the signal to the destination node. Diversity is obtained by means of two main characteristics of the wireless channel: its broadcast nature, and how it provides diversity through independent channels. In other words, Cooperative Communications is a method by which to obtain diversity without the use of multiple antennas in the transmitter or receiver. Currently, terrestrial Cooperative Communication employs two main methods: (1) DF (Decode and Forward), and (2) AF (Amplify and Forward).

$\mathrm{DF}$ is a rather complex technique that receives the signal, decodes it, and then processes the information, re-encoding the message that is then transmitted to the destination. AF is merely a simple amplification of the signal and retains its current bandwidth, coding and modulation. This concept must be transferred to satellite networks, but the characteristics of these techniques have to be re-evaluated to consider possible synchronization issues and bandwidth allocations. Another issue to examine is the selection of the relay terminals and whether they will be fixed terminals of mobile relays, portable by other subscribers. 
In this respect, the author proposes the use of Decode and Forward to relay the signal from the satellite channel [21] to the terrestrial network in order to extend the coverage area. Decoding the signal, although more complex in the relay design, allows for the adaptation of the terrestrial network bandwidth to the decode message, so it is unnecessary to preserve the bandwidth from the satellite link in the terrestrial application.

\subsubsection{Characteristics of the Satellite Channel Model}

The hybrid satellite/terrestrial channel will be considered to have a direct line of sight (LOS) from the satellite and several terrestrial receivers located in an open area, thus resulting in a propagation [22] model with several paths. The satellite LOS path is modeled using a Rician [23] distribution. The Rician distribution is a multipath model that is described by the factor $\mathrm{K}$, which is the ratio of the power in the direct link to the power of the multipath links. Typical values for $\mathrm{K}$ are $5 \mathrm{~dB}, 7 \mathrm{~dB}$, and $8 \mathrm{~dB}$. The terrestrial model is described as a Rayleigh distribution, which is a type of distribution in which the LOS is nonexistent, so $\mathrm{K}=0$. Rayleigh fading channels affect the signal much more that Rician fading channels because all the paths that reach the receiver are reflected, diffracted or from scattering. Other variables used to describe these multipath fading channels include delay spread and Doppler spread. The maximum Doppler shift can be found by the following formula:

$f_{d m}=\frac{v}{c} f_{0}$,

where $\mathrm{c}$ is the speed of light, $\mathrm{v}$ equals mobile speed, and $\mathrm{f}_{0}$ is the frequency. Some characteristics of the satellite channel include: (1) non-linear distortion introduced by 
onboard power amplifier; (2) long round-trip propagation time; (3) reduced time diversity; and (4) rain attenuation.

The High Power Amplifier (HPA) is introduced in the satellite channel. HPA operates near the saturation region to maximize output power and efficiency. Under the abovementioned condition, a non-linear distortion [24] is introduced, increasing spectral re-growth and in-band distortions. This can be problematic if higher order modulations are used, like 8PSK, 16QAM, etc. (see Figure 1.2). These also damage the channel capacity by increasing adjacent channel interference. Thus, a waveform pre-distorter is necessary to address these issues. Typically, HPAs are TWTs (Traveling Wave Tube).

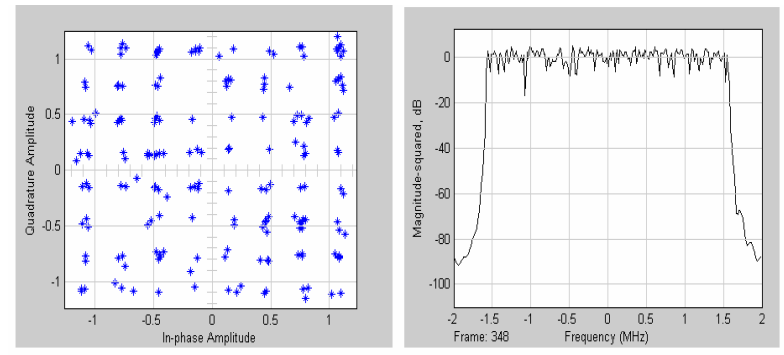

Fig. 1.2 64QAM Constellation and OFDM Spectrum

\subsection{Research Purpose}

This study aims to adapt the use of OFDM and Cooperative Communications to the satellite link, which will in turn be utilized by future $4 \mathrm{G}$ mobile systems. In this respect, the researcher will investigate and propose space and time diversity techniques that enable the use of OFDM in links with long delays, when the round trip obstructs the accuracy of the channel quality parameters and sounding signals that are exchanged between subscribers and base stations to maintain updated channel estimations. The link reliability must be improved by using these techniques. 
The development of new satellite systems will align with terrestrial networks, so that the two systems will be fully integrated, both offering high QoS and data rates. The researcher will investigate and propose a Decode and Forward technique that efficiently relays the data transmitted over the satellite link to a destination that is out of the reach of the satellite. MIMO techniques will also be investigated, as they can be integrated to improve the received signal quality. They can also increase data communication speed, using digital signal processing techniques to shape and combine the transmitted signals from multiple wireless paths created by through multiple transmit and receive antennas.

The end result of this study will be a set of tools that improve communication capacity, speed and performance. Furthermore, the study's findings will extend the network lifetime, increase the throughput and stability region for multiple access schemes, expand the transmission coverage area and provide cooperation tradeoffs beyond source channel coding for multimedia communications. Figure 1.3 shows the general image of a system in which a satellite transmits a signal to a mobile user who is out of reach. The terrestrial network relays the signal, using Decode and Forward, to the intended user.

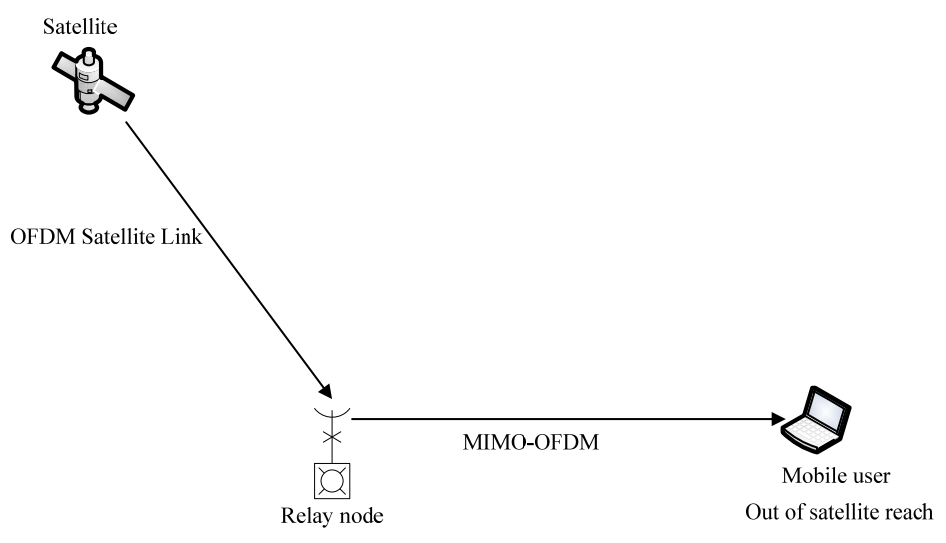

Fig. 1.3 OFDM and Cooperative Satellite Communications 


\subsection{Problem Statement}

Although OFDM has been known by the scientific community since 1966, it was only sufficiently developed for use in systems during the 1990s. OFDM offers a number of advantages over other modulation methods, but it also features some disadvantages. A system design using OFDM can only be implemented after considering a number of tradeoffs. Some necessary considerations in its use are as follows: (1) ability to operate without a classical channel equalizer, when communicating over disperse transmission mediums such as wireless channels. Meanwhile, it can accommodate the time and frequency domain channel quality variations of the wireless link; (2) channel quality fluctuations are averaged due to frequency domain spreading codes that lead to the concept of Multi Carrier Code Division Multiple Access (MC-CDMA). In this framework, only a few chips of the spreading code are obliterated by the frequency selective fading, and therefore, the spreading code and its data may still be recoverable; and (3) space-time coding technique that can reduce the channel quality variations of the wireless link. Space-time coding is also considered an anti-fading technique, which provides a high diversity gain.

The 3GPP group is currently working in the mobile area to develop Long Term Evolution (LTE) of UMTS (Universal Mobile Telecommunication System) radio access. 3GPP has adopted OFDM-based techniques in the design of the physical layer. In addition, they have proposed state of the art coding schemes and MIMO techniques in pursuit of higher system efficiency. Likewise, broadcast systems are adopting this trend. The DVB project decided to adopt OFDM-based MIMO, Low Density Parity Check (LDPC), and upper Layer FEC in the standards for DVB-T2. Thus, 3GPP LTE will 
clearly become an important factor in the transition to $4 \mathrm{G}$ mobile systems [25]. An effective method for the successful deployment of mobile satellite systems in $4 \mathrm{G}$ networks is the maximization of technology commonalities with those of the terrestrial networks. The terrestrial wireless interface can be adopted as the base line for the satellite radio interface, introducing some modifications necessary to handle the satellite channel.

An important characteristic of 3GPP LTE is its physical layer design. This design must be considered when dealing with satellite links. 3GPP lacks the large time interleaving that is necessary in long, fading channels, such as a satellite link. The techniques used by the 3GPP terrestrial links are not efficient in satellite channels, and alternatives are required to increase link reliability. This issue will be addressed by modifying the 3GPP physical layer, so the satellite link will be used efficiently. Increasing diversity by time and frequency coding is essential to this aim.

In this framework, another problem that arises is the transference of a service between a satellite link and a terrestrial, in which the bandwidth can be limited, so a simple AF technique is insufficient. Bandwidth allocation is a key parameter to consider when relaying a service; thus, a DF method will be necessary to adapt the data rate provided by the satellite to a sometimes limited bandwidth on the terrestrial link. This issue is addressed by using a bandwidth adaptation mechanism that works in conjunction with the DF technique. The relay node must decode the incoming signal, so that the data can be re-encoded again at a different rate and "accommodated" to the next link.

Distributed space time coding has the advantage of high diversity gains, with less spectral efficiency cost, when compared to repetition-based protocols. They function by 
having the multiple relays forward the received signal with a space time algorithm, similar to the multiple transmitter antennas of a space-time MIMO system.

\subsection{Research Questions and Hypothesis}

Following the problem statement, the questions below must additionally be investigated:

Question \#1: Does the proposed time diversity technique increase the satellite link reliability, so OFDM can be used to carry high data rates?

Question \#2: What modulation technique is most suitable to address satellite channel impairments, such as non-linear distortion effects of the satellite HPAs?

Question \#3: Is the hybrid satellite/terrestrial channel model (Rician/Rayleigh) accurate in most cases?

Question \#4: How is the bandwidth efficiency in DF techniques addressed in the relay nodes, which can be fixed or mobile?

Hypothesis \#1: Time diversity can adapt channel correlation in slowly varying channels. No additional complexity is needed at the receiver.

Hypothesis \#2: M-APSK modulation's hybrid constellation will adequately handle nonlinear distortion. Thus, in conjunction with a good error correction algorithm, it is perfectly suited for satellite transmission at high data rates.

Hypothesis \#3: Assuming that the mobile user has direct sight of the satellite and is operating at frequencies of the order of $4 \mathrm{GHz}$, a Rician model can be assumed to be correct for most cases. 
Hypothesis \#4: Re-encoding requires a more complex relay node. Furthermore, if these nodes are mobile, they require more efficient battery and processing power. If they are fixed nodes, they will be located in specific locations where they can cover areas like tunnels, in which access is difficult for both satellite and terrestrial networks. Adaptive mechanisms in Cooperative Communications can be bandwidth efficient.

\subsection{Significance of Study}

Successful transmission of high data rates over satellite links in $4 \mathrm{G}$ mobile systems using OFDM air interfaces will serve as an important tool for multimedia applications in particular, but also for data transmission. The researcher hopes the proposed OFDM satellite interface will be helpful in overcoming some of the difficulties faced in satellite links, using higher order modulation techniques such as M-APSK and M-QAM, in addition to addressing problems relating to long delays over such distances.

OFDM will not only provide clear advantages for physical layer performance, but also offers a framework for improving Layer 2 by proposing an additional degree of freedom. By using OFDM, it is possible to exploit the time domain, the space domain and even the code domain to optimize radio channel usage. This will ensure very robust transmission in multipath environments with few receiver complexities. 4G mobile systems will link satellite and terrestrial networks into one cooperative network that will access any user at any time and anywhere, delivering the services he needs. Transmission relays from satellite links to the terrestrial and areas poorly covered now will greatly impact economies and social structures. Any person or institution may access information, regardless of location. 
On the other hand the combination of OFDM in satellite links and Cooperative Communications will provide a continuous link that adapts the data rate sent by the source to the various bandwidths constraints. To this aim, the researcher anticipates that the use of Decode and Forward techniques for fixed relays terminals will enhance the coverage area of the system. Figure 1.4 shows a simplified diagram of a Decode and Forward scheme and how it will be used in future $4 \mathrm{G}$ systems.

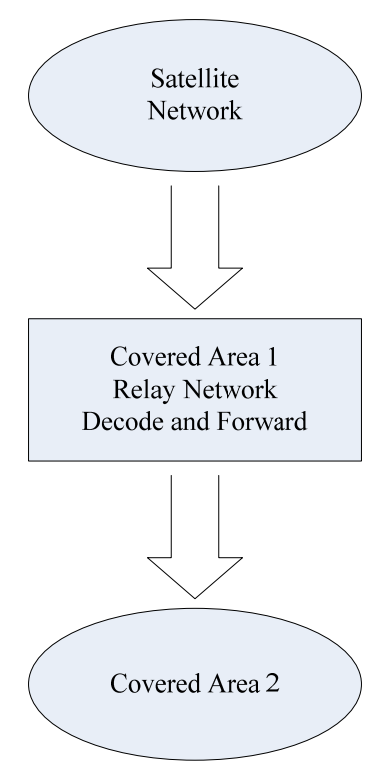

Fig. 1.4 Decode and Forward will adapt to Bandwidth Allocations

\subsection{Methodology}

Time diversity is a technique used in wireless communications to remedy errors that are present due to time-varying channel conditions. These errors can be caused by channel fading and a moving transmitter or receiver. Time diversity uses a retransmission of the data to solve such obstacles. OFDM presents a good solution for the satellite link, but one of its problems is its sensitivity to Doppler shifts. Thus, the time diversity technique may be a better solution to these issues. 
The orthogonal direction that OFDM offers allows for the division of incoming data into many sub-streams, so they can modulate several sub-carriers in a tight spectral space, thus heightening efficiency. The sub-carriers do not interfere with each other. An OFDM symbol is created by using the IFFT (Inverse Fast Fourier Transform). IFFT takes the spectrum and converts it into a time signal by multiplying it with a range of sinusoids.

Therefore, it is important to select an adequate modulation technique for both the satellite link and the terrestrial relay, M-APSK. Figure 1.5 presents the best satellite link characteristics, as mentioned in Section 4. M-APSK has a dual ring constellation, so the space between phases is better distributed than in M-QAM modulation. The ring can be viewed as a double ring of PSK constellation. Here, two parameters must be defined: (1) the relative radius of the outer PSK ring against the inner PSK ring, and (2) the relative angle between them.

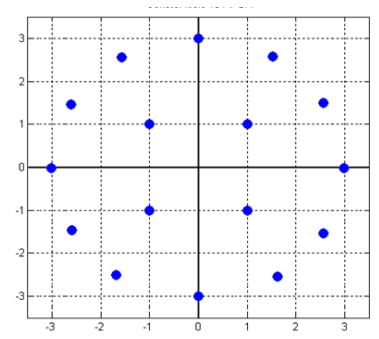

Fig. 1.5 16-APSK Constellation

The selection of the channel model is also very significant to the system architecture. A Rician multipath fading channel has a direct link between the satellite and the station, in addition to a number of multipath links. It is described by the factor $\mathrm{K}$ and the path loss, which is considerably large due to the long distance. An aspect yet to be decided is whether to use GEO, LEO or MEO satellites. 
In Rayleigh fading channels, the value of $\mathrm{K}$ equals 0 , so there is no direct link between the transmitter and receiver. This characteristic makes the model more cumbersome, and the received signal is a superposition of all the multipaths. A Decode and Forward technique is needed, particularly in this multipath channel model, so the error probability and the signal to noise ratio can be analyzed. SNR must be greater than the threshold value, depending on the type of error correction used after the decoding mechanism in each relay.

\subsection{Data Analysis and Preliminary Results}

Data analysis will be performed using a formal theoretical formulation of the system design, followed by simulations with MatLab Version 7.6 Communication Toolbox and Communication Blockset. The parameters to be analyzed are: frequency assignments, bandwidth allocations, frequency responses of the communication channel, constellation diagrams for the digital modulations, sub-carrier frequency and spacing (OFDM), fast Fourier transform size (OFDM), transmission time intervals, OFDM symbol times, forward error correction rates and iterations, bit error rates (BER), signal to noise ratios, and graphs of Eb/No versus BER.

The main parameter to be measured and analyzed is the probability of error at a given data rate and SNR. The parameter Eb/No, where Eb is the bit energy and No is noise energy, is changed by adding more noise to the communication channel. For a given $\mathrm{Eb} / \mathrm{No}$, the system is simulated and the corresponding BER is calculated. An example of a graph showing the $\mathrm{Eb} /$ No versus BER is shown below in Figure 1.6. 


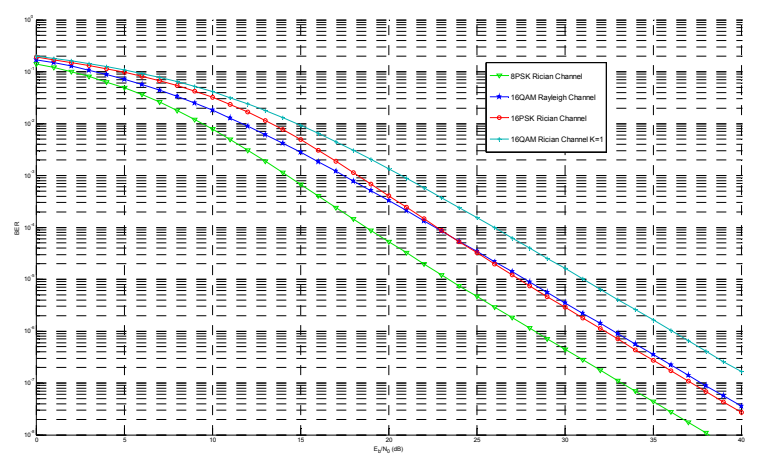

Fig. 1.6 Graph showing Eb/No versus BER

It is expected to reach an operating value for $\mathrm{Eb} / \mathrm{No}$ with strong results in its corresponding BER. BER is closely related to the system carrier-to-noise ratio $(\mathrm{C} / \mathrm{N})$, i.e., the signal SNR after the receiver filter, but before detection. As Eb/No increases, a better result in BER is expected, and vice versa. The objective, as stated before, is to find an optimal value of $\mathrm{Eb} / \mathrm{No}$ that is realistic for the modulation technique selected. The relation between $\mathrm{Eb} / \mathrm{No}$ and $\mathrm{C} / \mathrm{N}$ is given by the following equation:

$\frac{C}{N}=\frac{E_{b}}{N_{0}} \frac{D R}{B}$, where DR is the data rate and B is the signal bandwidth.

Some results of OFDM with 8PSK, 8QAM, 16PSK and 16QAM are shown in Figure 1.7. MatLab was used for the simulation with no FEC algorithms over an AWGN (Additive White Gaussian Noise) channel [3].
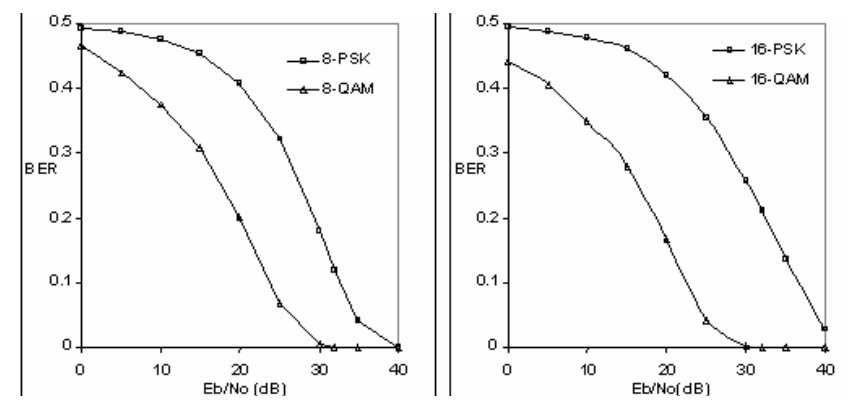

Fig. 1.7 Eb/No versus BER (no FEC) with PSK and QAM Modulation 


\subsection{Dissertation Organization}

The following chapters are organized as follows. Chapter 2 presents a survey of what studies have been conducted, and how, relating to $4 \mathrm{G}$ mobile systems. This includes what previous researchers have accomplished using OFDM and MIMO techniques. In addition, recent trends in Cooperative Communications are presented. Chapter 3 discusses channel models and presents hybrid architecture of the satellite/terrestrial communication link. Then, Chapter 4 covers the use of OFDM in $4 \mathrm{G}$ systems and satellite transmissions, as well as how to improve it in order to obtain reliable results in satellite channels. Chapter 5 analyzes MIMO techniques and how they can be used in the terrestrial link. Next, Chapter 6 introduces the concept of Cooperative Satellite Communications and analyzes an approach to transfer information from the satellite to the terrestrial link. Finally, Chapter 7 presents the dissertation conclusions and future research issues. 


\section{Chapter II. Literature Review}

Current studies of future $4 \mathrm{G}$ mobile systems focus on the use of OFDM as a way of providing spectrum efficiency, along with high data rate transfer. OFDM has proven to be very effective in terrestrial networks, so its potential use over satellite transmissions would guarantee $4 \mathrm{G}$ systems capable of covering all geographical areas. OFDM splits a carrier with large bandwidth into multiple orthogonal sub-carriers of much smaller bandwidths. Cooperative Communications has also been intensively investigated by researchers as a way to transfer information from the source to the destination, using relay nodes when the end user is unreachable by the source. Cooperative Communication is a fairly recent concept and offers a reliable solution when a communication channel is unavailable in a network setting. This chapter puts forth a review of the topics that have already been investigated in OFDM, Cooperative Communications and the challenges of migration to $4 \mathrm{G}$ systems.

\subsection{OFDM}

After more than 30 years of research and development performed in different institutes, OFDM has been implemented in high speed digital communication. By using Digital Signal Processing and advanced Very Large Scale Integration (VLSI), initial problems of OFDM implementations no longer exist. Turbo codes are binary error correcting codes built from the parallel concatenation of two recursive systematic convolutional codes, which use a feedback decoder. Turbo codes can achieve the performance of BER at close to the Shannon limit. The application of turbo codes together with OFDM is quite reliable because, with the appropriate interleaving, coded 
modulations can achieve a diversity effect through exploitation of the multipath nature of the fading channel.

One of the firsts attempt to use OFDM over a satellite link was presented by Fernando and Rajatheva [7], who wrote a very brief paper on the use of OFDM and turbo codes for LEO satellite channels in global mobile communications. The bit error rate performance for turbo-coded OFDM from different modulation schemes was considered. Then, the results were compared with Trellis Coded Modulation OFDM (TCM-OFDM) and Convolutional Coded OFDM (CC-OFDM), revealing the advantages and disadvantages of using TC-OFDM in these channels.

Doufexi, Armour, Nix and Beach [9] address the initial physical, one-layer performance results in a number of transition modes and channel scenarios in a cellular network using OFDM. Space time techniques were considered as a mean of enhancing the performance of the coded OFDM system. The OFDM provided data rates of $1.3-$ 12.5 Mbps by employing different transmission modes. The authors concluded that the system would be suitable for multimedia traffic, which is a key requirement for $4 \mathrm{G}$ mobile networks. In order to achieve diversity gains and to enhance performance, spacetime block codes were employed. Although this scheme has low complexity and is easy to implement, it provides considerable improvements in performance without any bandwidth expansion. Gains of between 9-14 dB were observed, depending on the transmission mode and channel scenario.

An MIMO-OFDM-based air interfaces system design was presented in [5]. The study focused upon various research topics relating to MIMO-OFDM-based air interfaces, including spatial channel modeling, a MIMO-OFDM transreceiver design, MIMO- 
OFDM channel estimation, space time techniques, and error correction codes. The linklevel simulation results showed that MIMO-OFDM is a promising means to future broadband wireless access. An MIMO system takes advantage of the spatial diversity obtained by spatially separated antennas in a dense multipath scattering environment. MIMO systems can be implemented in a number of different ways to obtain either a diversity gain to combat signal fading or to obtain a capacity gain.

Generally, there are three categories of MIMO techniques: (1) improve the power efficiency by maximizing spatial diversity; (2) use a layered approach to increase capacity; and (3) exploit knowledge of the channel at the transmitter. MIMO opens a new dimension, space, to offer the advantage of diversity and has therefore been adopted in various standards. The combination of MIMO and OFDM is indeed very attractive and has become a promising broadband wireless access technique.

A hybrid multicode interference cancellation (MCI) and minimum mean square error (MMSE) [10] detection scheme for turbo-coded OFCDM systems makes use of channel estimation, based on a code-multiplexed pilot channel. Hybrid detection outperforms pure MMSE detection in various channel conditions, especially for a high level modulation scheme. To carry out interference regeneration for the hybrid detection, the conventional turbo decoding algorithm, which only decodes systematic bits, should be extended to decode parity bits as well. Moreover, two iterations in turbo decoding are sufficient to provide a solid performance for the system with the multistage hybrid detection.

Turbo-coded OFCDM, with hybrid MCI cancellation and MMSE detection, shows the following three potential results. In the first result, the hybrid detection outperforms 
pure MMSE. The performance improves as the number of stages increases. For QPSK, the hybrid detection of two stages is sufficient. For 16QAM, on the other hand, the hybrid detection of five stages is necessary. Hybrid detection works more efficiently for 16QAM than for QPSK. In order to perform interference regeneration for hybrid detection in the second potential result, the conventional decoding algorithm of turbo codes, which only decodes systematic bits, must be extended to decode parity bits. Furthermore, in pure MMSE, at least four iterations are needed in the turbo decoding to provide stable performance, while only two iterations are enough in the hybrid detection. Finally, in the third potential result, the proposed method for MMSE weight estimation works well with proper values of the average window size in time-domain and in frequency-domain.

OFDM cooperative space-time diversity [12] realizes the theoretical benefits of space-time cooperation. The system is based upon a two-phase space-time cooperation protocol, as well as a transmitter and receiver architecture that facilitates cooperation. The authors devised a frame structure upon which they built practical timing and frequency synchronization algorithms and a channel estimation algorithm. They also validated the performance of the proposed synchronization and channel estimation algorithms through simulations.

Some of the simulation results are shown in Figure 2.1 below. These results have demonstrated that the CO-OFDM system achieves a diversity gain comparable to that of the conventional space-time coded OFDM, even when synchronization is taken into account. For a channel with little frequency diversity, the CO-OFDM system provides as much as a $6.7 \mathrm{~dB}$ energy gain over a single antenna SA-OFDM system at a target frame error rate of $10^{-2}$. 


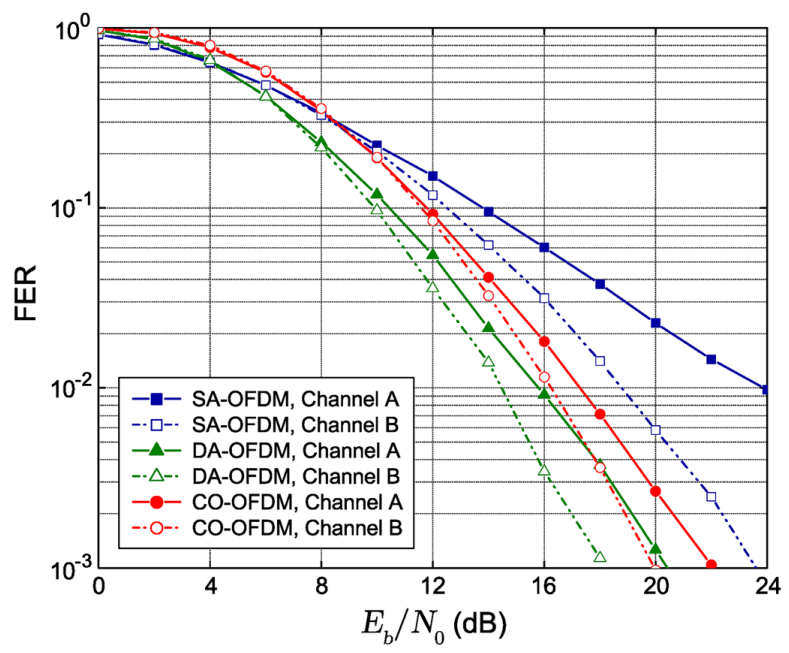

Fig. 2.1 Eb/No versus FER for Proposed Synchronization and Channel Estimation

These results indicate the improvement of CO-OFDM in terms of frame error rate (FER) over single antenna OFDM (SA-OFDM) and double antenna OFDM (DAOFDM). For a channel with little frequency diversity, when the proposed synchronization and channel estimation algorithms are employed, the CO-OFDM apparently provides as much as a $6.7 \mathrm{~dB}$ energy gain over an SA-OFDM system at a target FER of 0.01 .

Multi-user channel estimation in 4G OFDM [18] is a pilot-aided, multi-user channel estimation for a proposed $4 \mathrm{G}$ wireless system that employs OFDM in a synchronous SDMA environment, which can also be regarded as an MIMO environment. An Iterative Low Complexity (ILC) estimation technique for Time-Invariant (TI) channels that utilizes extrapolation and specially designed pilot sequences is presented.

Unlike most multi-user channel estimation methods in literature, the ILC method does not assume that the number of utilized sub-carriers is equal to the OFDM FFT size. Then, a channel interpolation method is described for time-varying (TV) channels. Simulation 
results show that the performance of their method equals that of current technologies, but the computational complexity is reduced.

The prospect of using OFDM in satellite communications [4] in 4G mobile systems is integral to current research trends and open issues in satellite communications. Its application in 4G Mobile systems and the link between satellite networks and terrestrial are also under discussion. The authors offer a characterization of the communication channel and the possible use of OFDM in the satellite link. A new concept of Cooperative Satellite Communication is also reviewed in this paper, focusing on the selection of Amplify and Forward or Decode and Forward techniques for the transition to terrestrial networks from the satellite link.

In MIMO-OFDM channels, if enough cyclic prefix (CP) is used, inter-symbol interference is completely cancelled, and consequently, spatial multiplexing interference (SMI) becomes the primary limiting factor in performance. The problem of the near optimum detection algorithm for MIMO-OFDM Channels [8] is approached by a joint detection algorithm. This algorithm is based on a new soft parallel interference cancellation (SPIC) technique, in which-on each iteration-a part of SMI proportional to the probability of correctness of estimation in the previous iteration is cancelled. The algorithm approaches the optimum performance in only a few iterations.

The algorithm is simulated for 4 states at $1 / 2$ turbo code and $8 \times 12,12 \times 12$, and $4 \times 4$ MIMO-OFDM channels, for different values of Eb/No. The BER and FER are both considered to be comparison criteria and demonstrate that its performance is good and very close to the interference free case. Between the transmitter and receiver six, exponentially decaying Rayleigh distributed paths are assumed. 
It is essential to use M-PSK and M-QAM of high orders in order to establish a reliable OFDM link. A comparative performance analysis of OFDM, using M-PSK and M-QAM modulation techniques [3] by Patel and Dalal, is presented in a paper. They compared both modulation and the results of BER measurements, showing that the abovementioned schemes used in the OFDM system can be selected on the basis of the required power or spectrum efficiency.

The comparison of M-PSK and M-QAM indicates that BER is larger in M-PSK when compared to M-QAM, and it generally depends on applications. For higher values of M (i.e., $\mathrm{M}>16$ ), the QAM modulation scheme is used in OFDM. However, Patel and Dalal do not consider the use of error correction algorithms.

\subsubsection{OFDM and Channel Correlation}

OFDM and channel correlation, using time-varying cyclic delay diversity [11], analyze the influence of time-varying cyclic delay diversity (TV-CDD) on the channel fading correlation properties in OFDM systems. CDD only increases the frequency diversity at the receiver; on the other hand, TV-CDD introduces additional time diversity, which can be exploited without the need for additional complexity at the receiver. Due to the increased frequency and time selectivity, an unintended higher channel estimation effort is possible. The authors analyzed the impact of choice upon the maximum cyclic delay, showing that the resulting channel for TV-CDD can be modeled as an uncorrelated Rayleigh fading channel (except for the first sub-carrier) for a significant maximum cyclic delay. Simulation results showed a potential choice of small time-varying cyclic delays to guarantee the standard conformability of the TV-CDD technique at the receiver, 
without significant performance degradations. They improved upon their previous work on time-varying cyclic delay diversity, performed in 2007.

\subsection{Cooperative Communications}

Cooperative transmissions, in which a source and relay cooperate to send a message to a destination, can provide spatial diversity against fading in wireless networks. Two methods of relaying are known as Decode and Forward (DF) and Amplify and Forward (AF). Decode and Forward is a more complex scheme that needs a powerful relaying mechanism, since the relay will decode the incoming signal and re-encode it again, before sending it to the destination. Amplify and Forward is much simpler, since the relay node only amplifies the signal it receives, before sending it to the destination node.

The performances of AF and DF in Rayleigh channels [14] done by Souryal and Vojcic are derived from analytical expressions of the error probabilities of AF, DF, and a new Hybrid AF/DF relaying protocol, for systems using strong forward error corrections in quasi-static Rayleigh fading channels. Their expressions compare favorably with simulation results using turbo codes. Analytical results include an exact expression for the distribution of the SNR in AF transmission. The protocols that achieve diversity with regard to the optimum position of the relays are midway between the source and destination, implying that mutual relaying to a common destination is suboptimal.

The hybrid scheme has the advantage of not requiring any feedback from the relay to the source. Furthermore, relative to AF, it avoids noise accumulation when the relay successfully decodes the source's transmission. The optimal combination of the relay and source's transmission in $\mathrm{AF}$ mode requires knowledge by the destination of the 
cumulative signal attenuation along the relay path. In order to combat synchronization errors in Cooperative Communications, OFDM was the most appropriate scheme. However, OFDM is very sensitive to carrier frequency offsets (CFO). For conventional non-cooperative communications, only one CFO is present, and its effect is easy to compensate. In Cooperative Communications, the CFO between each relay and destination may be significantly different. To solve this issue, a spaced-frequency coded Cooperative Communications [17] model was proposed. It is based upon a detection scheme for spaced frequency codes, which function much better than conventional SISO zero forcing (ZF) and minimum mean square error (MMSE) methods. Parallel Interference Cancellation (PIC) can be used to further improve system performance.

Simulations results showed that the proposed scheme, combined with PIC, is capable of achieving almost the same level of performance as cases without CFO (see Figure 2.2).

The researchers improved upon previous work that applied the OFDM technique with the intent of achieving full cooperative diversity and full multipath diversity for asynchronous cooperative communications, in which a type of high data rate spacefrequency (SF) codes was used at relay nodes.

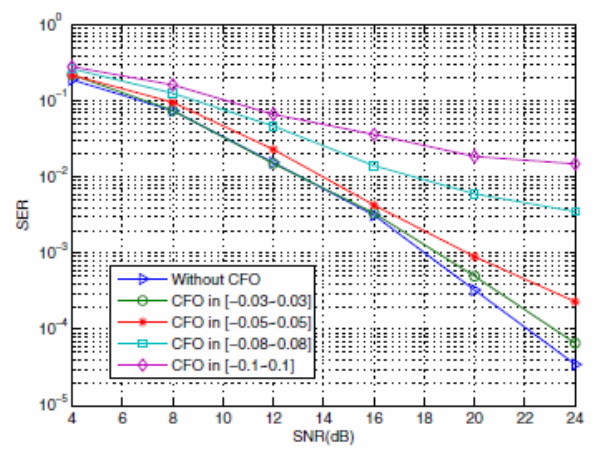

Fig. 2.2 SNR versus SER High Rate Spaced-Frequency Codes with Multiple CFO 
In Cooperative Communications, the study of symbol error rates (SER) is an important factor when investigating system performance. SER can be obtained for both DF and AF techniques.

Wu and Patzold [16] examine the SER performance in a cooperative communication system operating in AF mode, in which the channel state information (CSI) available at the receiver is an estimate of the channel gains with estimation errors. They derived the probability density function and the moment generating function of the instantaneous signal to noise ratio (SNR) at the destination terminal. These statistical quantities were then applied to study the performance of an AF-cooperative communication system. Thus, an accurate SER expression for AF cooperative communication systems with channel estimation errors was obtained. This study's result encompasses the performance of AF cooperative communications systems with perfect CSI as a special case. Furthermore, simulation results were presented to verify the correctness of the analytical solutions. The researchers considered for their simulations an AF cooperative communication system, with quadrature phase-shift keying (QPSK) modulation for different levels of channel estimation errors.

Another factor to be considered in a cooperative system is the throughput. In [15], the authors present a cooperative communication approach called Extended Communications using Helper Organizations (ECHO) that can increase throughput, communications range and covertness. A cooperative communication link such as ECHO (see Figure 2.3) is difficult to geo-locate because it appears as multiple, spatially-distributed, low power transmissions, rather than a single high-power source, as in conventional point-to-point communications. The author uses the Information Theory and Estimation Theory to 
present a theoretical framework for quantifying expected performance gains. ECHO can be used in Sensor Networks, MANETs, SATCOM and ground-based communications. A cluster of satellites can cooperatively relay information to the ground, enabling a node in the cluster to tap into the aggregate capacity of the satellite cluster.

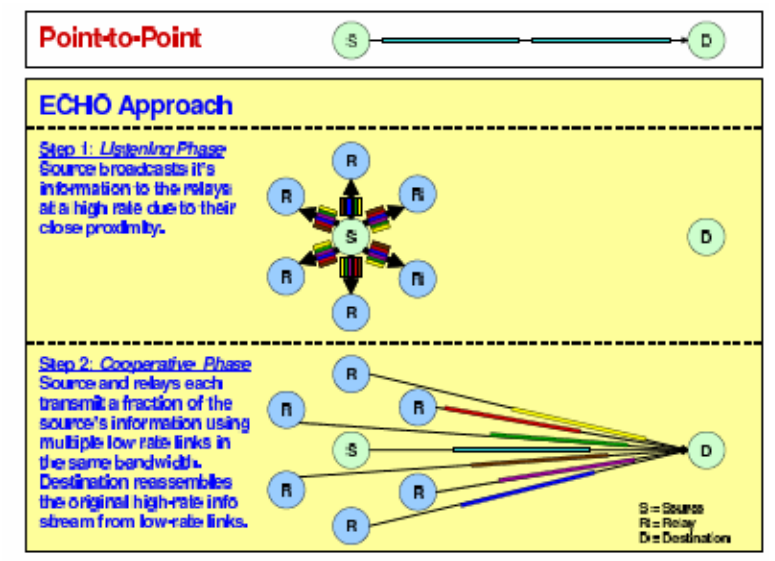

Fig. 2.3 ECHO

MIMO techniques can be combined with Cooperative Communication to increase the overall diversity gain. A MIMO and Decode and Forward mechanism is analyzed in [13]. Two types of new decoding algorithms for a network coding aided relaying (NCR) system are proposed, which adopt multiple antennas at both the transmitter and receiver. In NCR systems, the relay station (RS) decodes the data received from both the base station, as well as from the mobile station, and then combines the decoded signals into a single data stream, before forwarding it to both.

The authors considered the real case of obtaining decoding errors at the relay station, which results in erroneous forwarded data. They derived decoding algorithms for both the base station and the mobile station in order to reduce the deleterious effects of imperfect decoding at the relay station. The simulation results showed that the proposed algorithms 
provide substantial performance improvements, in terms of the attainable packet error rate, as a benefit of their more accurate error model.

\subsection{Challenges in Migration to 4G Mobile Systems}

Due to the rapid development of wireless communications networks, the launch of fourth generation mobile systems is expected in the next years. These systems focus on seamlessly integrating existing wireless technologies, including GSM, wireless LAN, and Bluetooth. This differs with $3 \mathrm{G}$, which barely focuses on developing new standards and hardware. Instead, 4G systems will support comprehensive and personalized services, providing stable system performance and quality services. However, migrating current systems to $4 \mathrm{G}[6]$ presents enormous challenges.

Researchers are currently developing viable frameworks for future $4 \mathrm{G}$ networks. Different research programs, such as Mobile VCE, MIRAI, and DoCoMo, possess their own visions of $4 \mathrm{G}$ features and implementations. Some key features of $4 \mathrm{G}$ networks are: high usability, anytime, anywhere, and with any technology; support for multimedia services at low transmission costs; personalization; and integrated services. Fourth generation networks are all IP-based and heterogeneous, allowing users to access systems anytime and anywhere. Users carrying an integrated terminal can also employ a wide range of applications.

However, in order to migrate current systems to $4 \mathrm{G}$ with the features mentioned above, a number of challenges must first be addressed. These include multimode user terminals, wireless system directories and selection, terminal mobility, network infrastructure and QoS support, security and privacy, fault tolerance and survivability, 
multiple operators and billing systems, and personal mobility. Some of these challenges have been extensively studied, such as multimode user terminals, wireless system discovery, terminal mobility, and QoS support. On the other hand, other topics require more attention, including wireless system selection, security failure, and survivability. Furthermore, addition research regarding implementations of personal mobility, billing, and accounting systems are also needed in $4 \mathrm{G}$ networks.

Satellite communications are not immune to this wave of innovation [4]. In March 2006, during the DVB forum after the publication of the DVB-S2 standard and the approval of DVB-SH specifications, the Commercial Module approved the mission of extending DVB-RCS to mobile scenarios, yielding the DVB-RSC+M mode. The objective of this act was to provide broadband interactive services to mobile users located on aircrafts, boats, and such vehicles as high speed trains, buses, and cars.

\subsubsection{Channel Models}

Typically, models for broadband satellite channels are referred to as having $\mathrm{Ku}$ (10$12 \mathrm{GHz}$ ) and higher frequency. They include those effects that are also found in fixed broadband channels, such as atmospheric attenuation, and those derived from mobility conditions, such as large and small scale fades, respectively, caused by obstacles much larger than the signal wavelength and by irregularities of such obstacles, typically resulting in well-known multipath propagation phenomena. Typical values of the coherence bandwidth for outdoor environments range from 7-11 MHz at the L-Band and are approximately $30 \mathrm{MHz}$ at the EHF Band, considering mobility effects. Frequency non-selective models have been thus far normally used for Ku-Band and Ka-Band 
signals, carrying data rates of up to several tens of Mbps. This subject is part of an ongoing investigation by the European Space Agency (ESA) through dedicated measurements.

Under the condition of no frequency selectivity, the large-scale effects are normally modeled with Markov chain, which determines the transition among channel states, and the small scale fades within each state are modeled by suitable statistical distributions, such as Rician and Rayleigh models. Four mobile environments are divided into categories of maritime, railway, and land vehicular. Maritime and railway mobile channels can be modeled as a single state Rician channel, with a high $\mathrm{K}$ factor. For land vehicular channels, on the other hand, narrowband Markov models for $\mathrm{Ku}$ and $\mathrm{Ka}$ are under investigation.

\subsubsection{Channel Coding}

State of the art technology in channel coding and modulations has been traditionally used by deep space communications, in which a robust error correction algorithm is needed due to large signal losses. Such losses decrease the receiver power, and the error correction provides large coding gains. Since the 1960s, there has been a shift from using convolutional codes to the use of concatenated codes. Concatenated codes employ both convolutional codes and Reed Solomon Block codes, thus strengthening the error correction mechanism.

The discovery of turbo codes and the rediscovery of Low Density Parity Check Codes (LDPC) in 1996 allowed for deep space communication with cutting edge technology. However, these new codes have been widely used in other applications as well, e.g., near- 
earth observation missions, when high date rates are required. Turbo codes and LDPC Codes base their functionality upon two principles: (1) a simple encoder with a predefined structure that is capable of constructing long code words with good Hamming distance characteristics, using a large interleaver and a parity check matrix; and (2) use of low complex techniques to decode the code words constructed by the encoder. The techniques are based on feedback or iterative decoding, which provide near optimal maximum likelihood performance.

Advanced channel coding is state of the art technology that has been used in satellite communications, in which a reliable bit error rate is needed, along with high data rates. The satellite services that use these techniques provide broadcasting services to fixed terminals in the $\mathrm{Ku} / \mathrm{Ka}$ Bands. They use the DVB-S2 uplink and DVB-RCS downlink. 


\section{Chapter III. Hybrid Satellite-Terrestrial Channel Model}

Different channel models have been used to describe the behaviors of communications channels. In this chapter, the author introduces a hybrid architecture that describes the satellite channel and the terrestrial channel. The author presents an approach of how such a channel can be modeled to be employed in future $4 \mathrm{G}$ mobile systems. This model serves as the basis for the next chapters' simulations and results.

\subsection{Wireless Channel Models}

The primary difference between the wireless channel model and the rest of the communication models is that in the former, the channel changes with time. Wireless channels are multipath fading channels, in which the signal that reaches the receiver changes in time and travels in different paths. Therefore, the signal experiences various phenomena, including reflection, diffraction, and scattering. The signal at the receiver is the sum of the contributions of all the above paths. Furthermore, there may be a direct path from the transmitter to the receiver, which is called the line of sight (LOS) (see Figure 3.1).

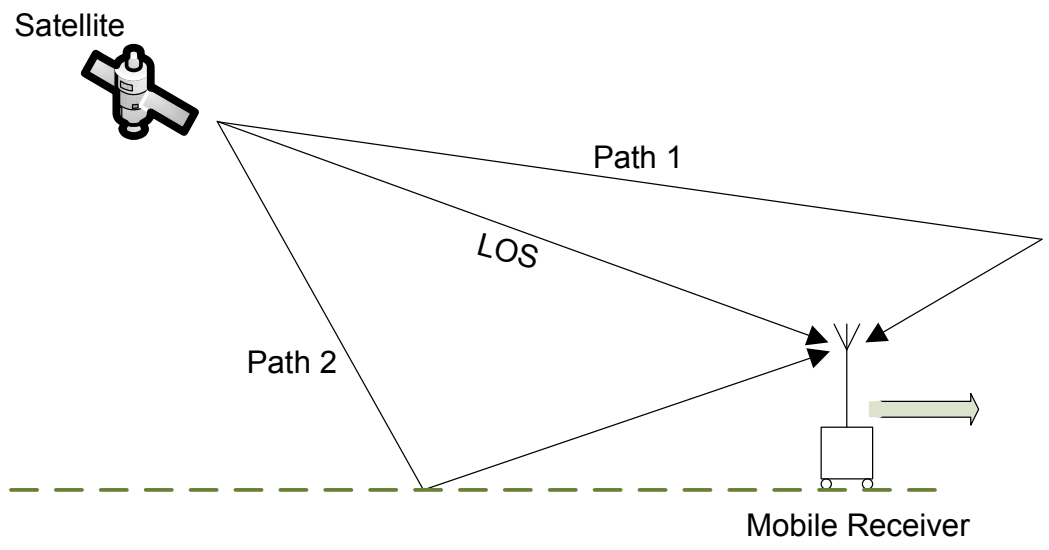

Fig. 3.1 Different Paths in a Wireless Link 
In the case that there is only a direct path from the transmitter to the receiver, the signal strength at the receiver decreases by $G \sim 1 / d^{2}$. With the contribution of all the paths in Figure 1, the signal strength at the receiver decreases by $G \sim 1 / d^{u}$, where $4>u>2$. The signal varies in time because the obstruction may or may not be present. This variation of $G$ is known as the channel variance. The signal at the receiver can be expressed as follows:

$y[n]=a_{0} x[n]+a_{1} x[n-1]+a_{2} x[n-2]+\ldots .+N[n]$,

where $a_{i}$ is the attenuation, and $[n-i]$ is the delay of the $i t h$ time instant.

Multiple paths provide a linear filter channel. Filter coefficients are $\left[a_{0}, a_{1}, \ldots ., a_{n}\right]$. All these channel coefficients change over time; with a multipath channel, the filter coefficients also change over time. There are some specific parameters that specify how fast the channel changes, or to what extent the multipath is present. The length of the channel impulse response is called the delay spread. As seen above, a channel can be modeled as a linear filter, depicted in Figure 3.2.

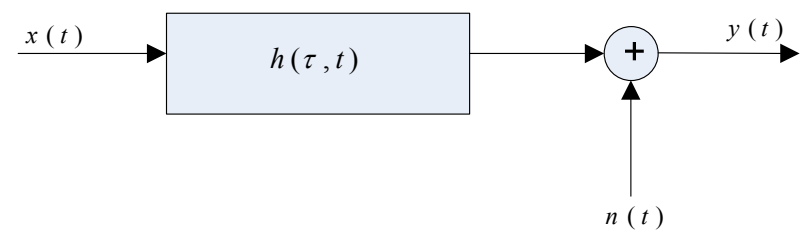

Fig. 3.2 A Channel Model Using a Linear Filter

The delay spread is the values for which the function $h(\tau, t)$ is not zero. From Figure 3.1 , it can be observed that if the receiver moves to the right, the signal received from the path to the left of the mobile receiver will travel a longer path as the receiver moves, thus 
decreasing the frequency. The paths to the right of the receiver will decrease, and thus, the frequency will increase. This phenomenon is known as the Doppler shift and is one of the more significant impairments that affect mobile antennas. Figure 3.3 shows the effects of the Doppler shift in a transmitted frequency.
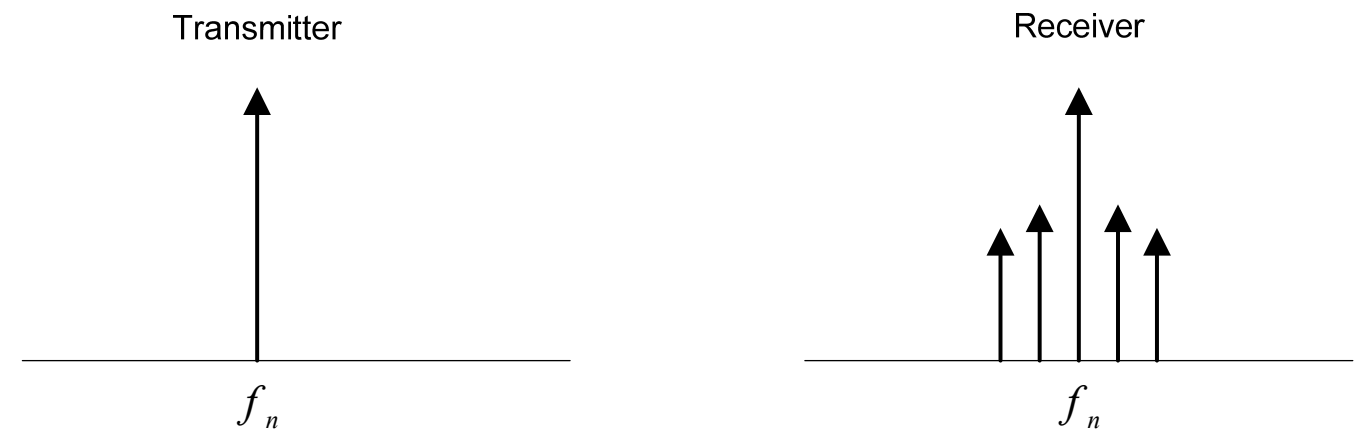

Fig. 3.3 Doppler Shift in a Single Frequency

If a single frequency is transmitted, a single frequency is expected to be received. Instead, due to the relative movements of the transmitter and receiver, the received frequency will spread in the frequency domain. The extent to which the frequency spreads is known as the Doppler spread. The frequency increases along some paths and decreases along others.

The degree of this spread is also a measure of how fast the transmitter and receiver are moving with respect to one another. The Doppler spread thus relates to the coherence time, which denotes the time interval after which the channel response is independent of the previous time.

As demonstrated above, wireless channels can be a function of time. Furthermore, they depend on the frequency and the receiver's position in space. Correlation is a measure of dependency between random variables. Increasing the correlation also 
increases the predictability. The autocorrelation and the power spectral density (PSD) are related in the Weiner-Khintchine theorem [51]. The PSD and the autocorrelation process are Fourier transform pairs. Table 3.1

Table 3.1 Autocorrelation and PSD pairs

\begin{tabular}{|c|c|}
\hline$\underline{\text { Autocorrelation }}$ & $\underline{\mathrm{PSD}}$ \\
\hline Time & Doppler Spectrum \\
\hline Frequency & Delay Spectrum \\
\hline Scalar Space & Wave-number Spectrum \\
\hline Vector Space & Wave-vector Spectrum \\
\hline
\end{tabular}

As a function becomes narrower in one domain, its Fourier transform pair becomes wider in the other domain, meaning that the PSD width can give us information regarding the channel coherence behavior.

As the PSD widens in the transform domain, its autocorrelation becomes narrower, and coherence decreases. The most common definition of the channel PSD width is its root mean square (RMS) width. RMS widths may be defined for PSD as a function of delay, Doppler or wave-number. An analysis of each function follows. Firstly, a large delay spread implies increased frequency selectivity and smaller coherence bandwidth. Secondly, an increased Doppler spread implies a channel with faster temporal fluctuations and smaller coherence time. Finally, an increased wave-number spread implies a channel with more rapid spatial fluctuations and a smaller coherence distance.

The channel duality principle states: the stochastic wireless channel as a function of (time, frequency, space) displacement may be characterized by an autocorrelation 
function. The Fourier transform of the autocorrelation function produces a (Doppler, delay, wave-number) spectrum. The width of this spectrum is characterized by its RMS (Doppler, delay, wave-number) spread. As this spread increases, the (temporal, frequency, spatial) selectivity of the channel increases, and the coherence (time, bandwidth, distance) decreases (see Table 3.2 below).

Table 3.2 Channel Duality Relationships

\begin{tabular}{|c|c|c|c|}
\hline & Time & Frequency & Space \\
\hline Variable & $t$ & $f$ & $r$ \\
\hline Coherence & $T_{c}$ & $B_{c}$ & $D_{c}$ \\
\hline Spectral domain & Doppler, $\omega$ & Delay, $\tau$ & Wave-number, $k$ \\
\hline Spectral width & $\begin{array}{l}\text { Doppler spread, } \\
\qquad \sigma_{\omega}\end{array}$ & $\begin{array}{c}\text { Delay spread, } \\
\sigma_{\tau}\end{array}$ & $\begin{array}{l}\text { Wave-number spread, } \\
\sigma_{k}\end{array}$ \\
\hline
\end{tabular}

\subsection{Satellite Channel Model}

Propagation characteristics in satellite channels are more susceptible to weather impairments, especially at higher frequencies [53,54]. Average rain and shadowing may completely disrupt the communication link. A mobile satellite channel model that takes into consideration potential weather impairments and the multipath-fading phenomenon is necessary in order to represent the satellite channel. Some models have been proposed, but they only consider one of either the multipath effects or the weather effect. The propagation effects present in a mobile satellite link include those related to the 
troposphere (rain, etc.), or effects caused by the receiver's environment (multipath). The troposphere effects are denoted by $\alpha$, and the environmental effects re denoted by $\beta$. The two effects are assumed to be statistically independent because their underlying mechanisms are independent. The amplitude of the received signal can be described as: $A=\alpha \cdot \beta$

The satellite channel model has two states (good and bad states): one is a nonshadowing state, and the other is a shadowing state $[52,56]$. This two-state model forms a Markov model. In the non-shadowing state, the received signal amplitude can be described as a Rician distribution:

$$
p_{\text {non-shadowing }}(A)=2 K \cdot A \cdot \exp \left[-K\left(A^{2}+1\right)\right] \cdot I_{0}(2 K \cdot A)
$$

where $K$ is the Rice factor.

In the shadowing state, where no LOS exists, the channel is described as a Rayleigh multipath fading. The signal at the receiver is expressed as:

$$
p_{\text {shadowing }}\left(\frac{A}{s_{0}}\right)=\frac{2 A}{s_{0}} \exp \left(-\frac{A^{2}}{S_{0}}\right)
$$

The envelope $s_{0}$ has a lognormal distribution.

The two states are time-sharing and can be modeled as a Gilbert model. If avg. is the average duration of the bad state, the probability density function at the receiver is:

$$
p_{\beta}(A)=(1-\text { avg. }) p_{\text {non-shadowing }}(A)+\operatorname{avg} \cdot p_{\text {shadowing }}(A)
$$

Of all potential weather impairments, rain is the most critical, especially in tropical weather, where rainfall can be severe. The long-term statistics of potential rainfall can be described by a lognormal equation: 
$P_{L}(L)=\frac{1}{\sigma_{d} L \cdot \sqrt{2 \pi}} \exp \left\lfloor-\frac{\left(\ln L-m_{d}\right)^{2}}{2 \sigma_{d}{ }^{2}}\right\rfloor, L \geq 0$

Studies on rain attenuation between fixed and mobile systems show that the probability distribution of the envelope of a mobile receiver can be described by the one used for the fixed system, multiplied by a factor that changes between 0.5 and 2.0 and is independent of rain attenuation [57].

Given that the impairments due to atmospheric and environment are independent [55], the probability density function of the signal amplitude $A=\alpha \cdot \beta$ is described as:

$p_{T}(A)=p_{\beta}(A) \cdot p_{\alpha}(A)$

$p_{\alpha}(A)=\frac{1}{\sigma_{\alpha} \sqrt{2 \pi}} \exp \left[-\left(A-m_{\alpha}\right)^{2} / 2 \sigma_{\alpha}^{2}\right]$

$p_{\beta}(A)$ and $p_{\alpha}(A)$ are the probability density functions of the mobile fading and weather impairments. Fig. 3.4 demonstrates the probability density function versus the amplitude.

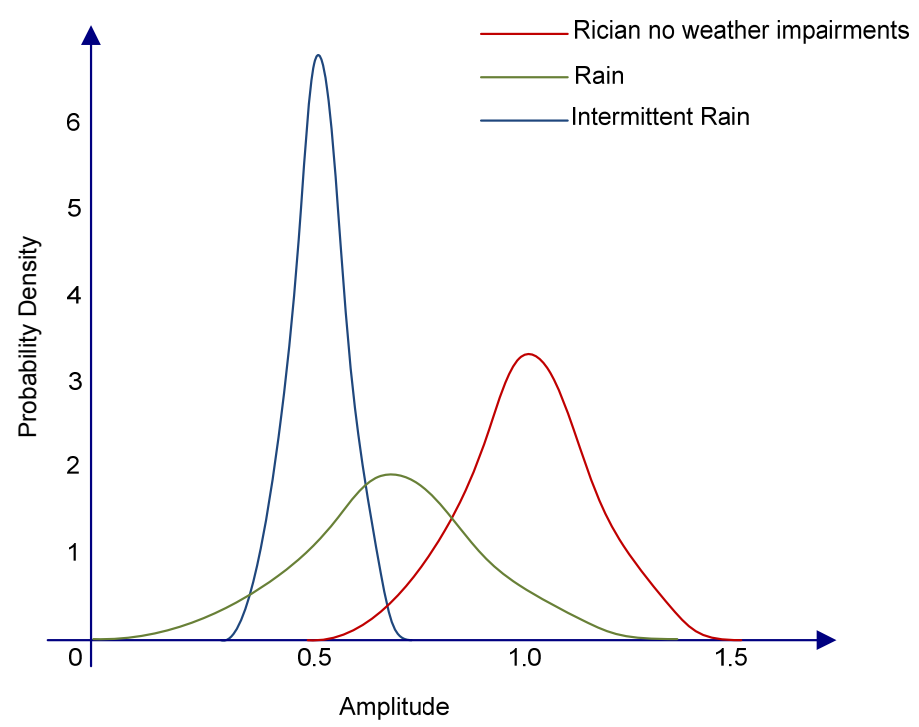

Fig. 3.4 Probability Density Functions for Non-shadowing State 


\subsection{Terrestrial Channel Model}

In the terrestrial channel, the signal also travels many paths. The signal reaching the mobile user is formed by the sum of these different paths. The terrestrial channel is formed in this analysis by the relay node and the mobile user, which will be explained in further detail in Chapter 6. This section instead focuses on the terrestrial channel model. As mentioned above, the signal reaching the receiver is the sum of many constructive and destructive paths. The receiver perceives this as a variation of the amplitude, phase and angle of the arrival signal. The received signal will be a set of attenuated, time-delayed, phase-shifted replicas of the transmitted signal. Fading is divided in two groups: (1) large-scale fading, which means variations over large distances, and (2) small-scale fading, including the effects of small changes in the separation between the transmitter and receiver. These variations can be caused by the mobility of the transmitter, the receiver or the intermediate objects in the path of the signal [58]. A non-deterministic model for the phenomenon exists and is thus characterized statistically. If there is no direct LOS between the transmitter and receiver, the Rayleigh distribution approximates the channel envelope, and the fading is known as Rayleigh fading. Figure 3.5 shows a model representation with a transmitter antenna and a mobile user.

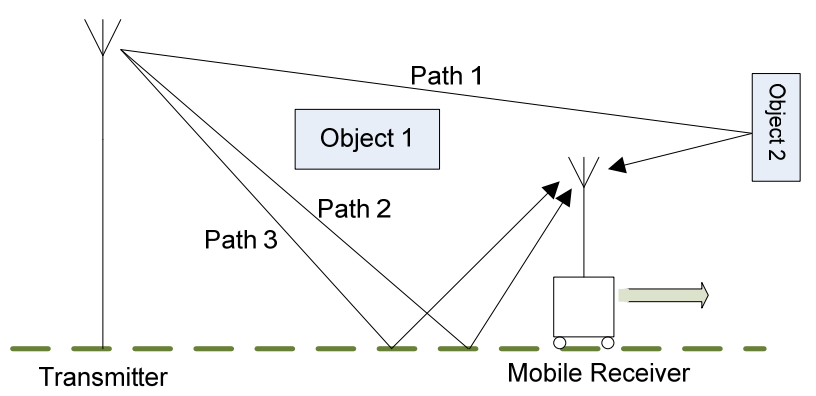

Fig. 3.5 Terrestrial Rayleigh Model 
Small-scale fading can be separated into two types - frequency selective fading and frequency non-selective fading. Frequency non-selective fading is also known as flat fading because all frequency components of the transmitted signal are affected by the channel response. In flat fading, the effect of the channel over the transmitted signal can be represented through a low pass equivalent of the channel response [60].

$$
h(t)=h_{\text {phase }}(t)+j h_{\text {quadrature }}(t)
$$

The phase and quadrature components are independent of each other and Gaussiandistributed. The envelope of the fading channel is Rayleigh-distributed, expressed as:

$r(t)=\sqrt{h^{2}{ }_{\text {phase }}+h^{2}{ }_{\text {quadrature }}}$

The instantaneous SNR at the receiver, $\operatorname{SNR}(t)$, is used as an indicator of the channel state at a given time. When the channel is poor, the signal will be severely degraded over the channel and the SNR will be low. Conversely, when the channel is strong, the signal can be detected easily at the receiver; thus, its SNR will be high. The instantaneous SNR is related to the channel response as follows:

$S N R(t)=|h(t)|^{2} \frac{E_{S}}{N_{O}}$,

where $E_{S}$ is the average energy per symbol, and $N_{O}$ is the noise power spectral density. If the noise is Additive White Gaussian Noise (AWGN), then $S N R(t)$ is exponentially distributed for Rayleigh channels.

The coherence time of the channel is the time over which the channel response can be considered invariant, given by the following equation:

$$
T_{C}=\frac{1}{f_{D}},
$$


where $f_{D}$ is the Doppler frequency, the maximum frequency shift due to the relative movement of the transmitter and receiver.

The autocorrelation function represents the variability of the channel over time. It depends on the propagation geometry, the velocity of the mobile and the antenna characteristics. The isotopic scattering is commonly assumed, meaning that the channel consists of many scatterers that are densely packed with respect to the angle.

This is usually referred to as the "classical model." The channel impulse response is a wide-sense stationary (WSS), and the continuous time autocorrelation function (ACF) of the phase and quadrature components does not depend on time, but on the time difference, $\tau$.

$R_{h_{\text {phase }}}(\tau)=R_{h_{\text {quadrature }}}(\tau)=b_{0} \cdot J_{0}\left(2 \pi \cdot f_{D} \cdot \tau\right)$

$J_{0}$ is the zeroth order Bessel function of the first kind, and $f_{D}$ is the maximum Doppler frequency.

In the discrete time domain, it is better to express the Doppler frequency normalized by the sampling rate, multiplied by the symbol period $T_{S}\left(f_{D} \cdot T_{S}\right)$. Low values of $\left(f_{D} \cdot T_{S}\right)$ indicate high correlation in the signal, and conversely, high values of the product $\left(f_{D} \cdot T_{S}\right)$ mean low correlation in the signal. In the limit $\left(f_{D} \cdot T_{S}\right) \rightarrow \infty$, there is no correlation, and the samples are independent of one another. If the envelope is a Rayleigh, the expression of the ACF can be defined as:

$$
R_{r}(\tau)=\frac{\pi \cdot b_{0}}{2} F_{1}\left(-\frac{1}{2},-\frac{1}{2} ;\left(J_{0}\left(2 \pi \cdot f_{D} \tau\right)\right)^{2}\right)
$$




\subsection{Hybrid Satellite-Terrestrial Channel Model}

The general characteristics of wireless channel models have been described, particularly the Rician and Rayleigh models. The approach put forth in this dissertation requires a hybrid model that combines the satellite link and the terrestrial in one channel to be used in $4 \mathrm{G}$ systems. This model needs to be one that, when simulated, takes into consideration the impairments presented in both links.

One must first analyze the satellite link to determine which model is most suitable. Since the relay nodes to be selected (refer to Chapter 6) are fixed nodes, their locations can be selected. On the other hand, if geostationary satellites (GEO) are used, this type of satellites has the property of being fixed with respect to the earth's rotation, so neither the satellite nor the earth station will move with respect to each other. Thus, it is guaranteed that there will be always a line of sight between the satellite and the earth station, thus resulting in a Rician model.

From the above analysis, the satellite channel can be modeled as having a direct line of sight and another multipath due to the reflections or scattering in the ground or nearby objects. This Rician model, when used in a simulator such as MatLab, must be concatenated with a propagation loss block that represents the signal attenuation caused by atmospheric conditions and long signal paths.

For the terrestrial model, however, the analysis is different. In the terrestrial link, the relay node is fixed, but the destination user is mobile, the movement of which is unpredictable, and therefore enters geographical areas out of the line of sight of the terrestrial transmitter [59]. Many times, the mobile user will be in areas that only signal 
paths, due to reflection or scattering, can reach. Therefore, a Rayleigh model is adequate to describe the terrestrial link.

Fig. 3.6 shows the architecture of this proposed hybrid model. The relay node is composed of the satellite receiver antenna, which feeds a processing unit (P.U.) in which all the decoding and further signal processing will take place, before the version of the received signal is handed to the terrestrial transmitter antenna for the interface with the terrestrial link.

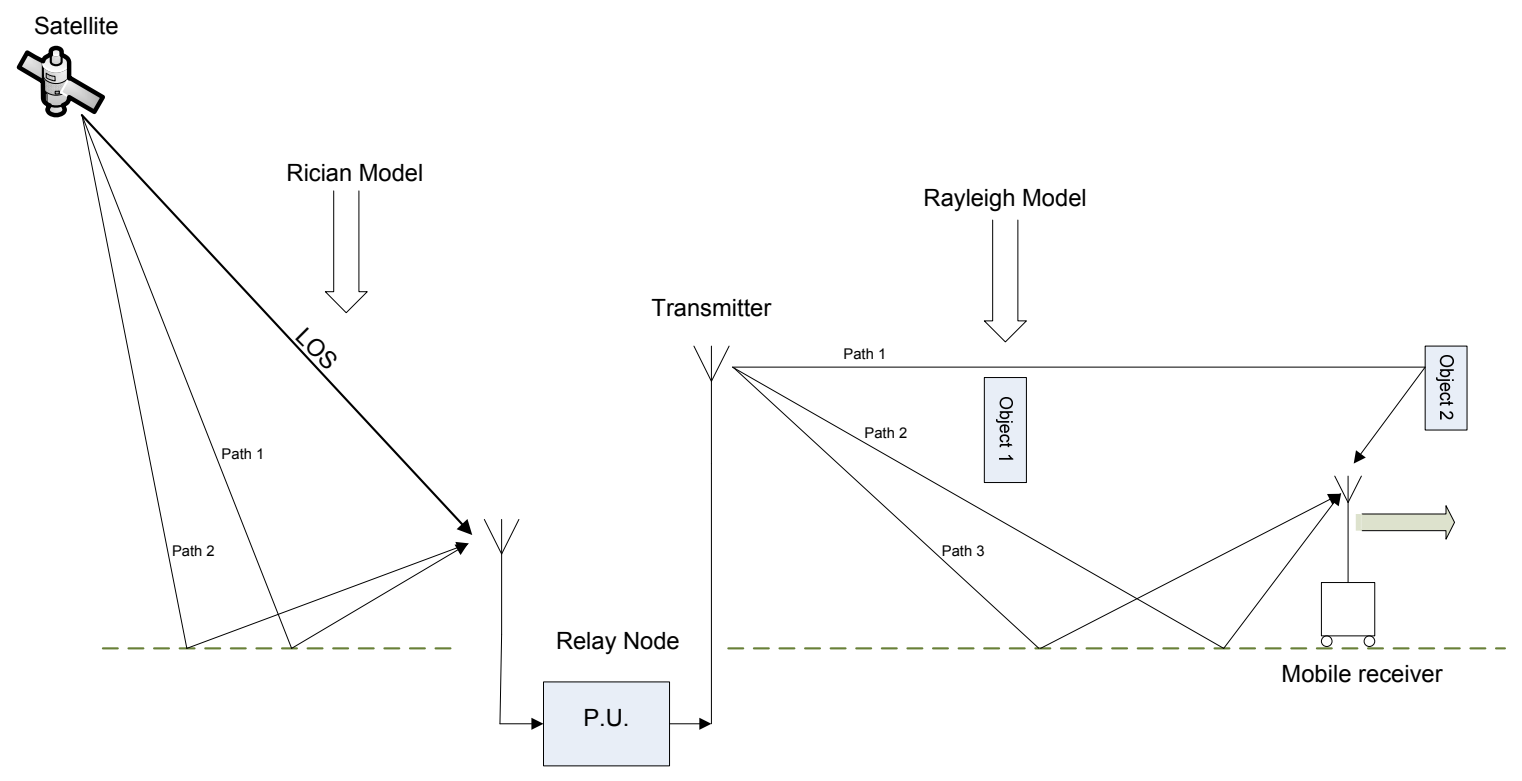

Fig. 3.6 Hybrid Channel Model Architecture

The P.U. will decode and forward the signal and accommodate the bandwidth of the received signal to the available bandwidth in the terrestrial link. A new modulation scheme may be necessary, if the terrestrial bandwidth is smaller than the satellite bandwidth. In the following chapters, each one of the channels will be analyzed, when used with higher order modulation and OFDM schemes; furthermore, an analysis will be completed to determine how the architecture can be used in Cooperative Satellite 
Communications to allow for the transfer of data from satellites to mobile users who can move out of the satellite's reach.

\subsection{Summary}

This chapter presented an analysis of wireless communication channel models and the variables that define them. The relations between random variables and their respective Fourier pairs have been examined, in addition to how they can be used to describe the

multipath channels. A satellite model was proposed in which there exists a direct line of sight between the satellite and the terrestrial node. A terrestrial model was also presented to model the terrestrial channel as a Rayleigh multipath fading.

The last section deals with a proposed hybrid architecture of a satellite-terrestrial channel, in which a P.U. is used to adapt the signal received from the satellite to the terrestrial channel. In the following chapters, each channel in particular will be analyzed, and Chapter 6 contains a combination of both in an approach to Cooperative Satellite Communications. 


\section{Chapter IV. OFDM Time Diversity in Satellite Channels}

This chapter covers an overview of discrete time signals and Discrete Fourier Transform (DFT). The principles of operation of OFDM are also analyzed. An approach is offered regarding how OFDM combined with time diversity can be effectively used in satellite channels with long delays. The last two sections deal with higher order modulation and the use of pre-distortion techniques in satellite links operating close to saturation.

\subsection{Discrete Time Signals and DFT}

A discrete time signal is a sequence $(\ldots x|-2|, x|-1|, x|0|, x|1|, x|2| \ldots)$, where $x|k|$ can be either real or complex. $\mathrm{x}|\mathrm{k}|$ is a sample of the sequence, i.e., $(\ldots 0,0, \mathrm{x}|0|=4,2,-2,3 \ldots)$.

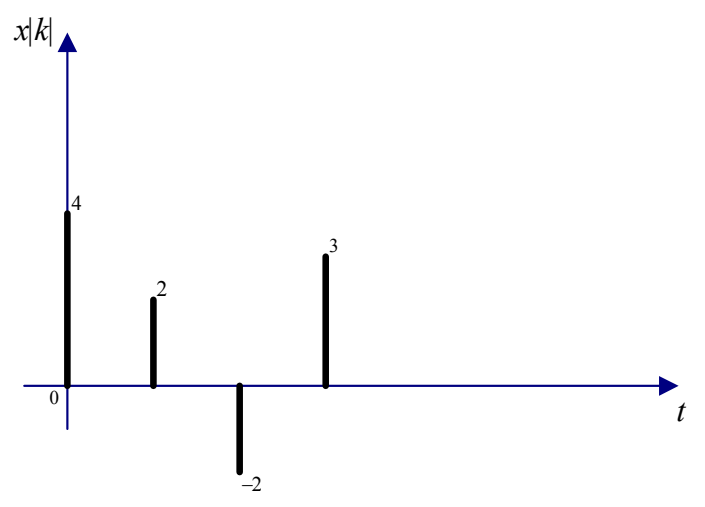

Fig. 4.1 Discrete Time Signal

For any given discrete $\mathrm{x}[\mathrm{n}]$ signal, the DTFT (Discrete Time Fourier Transform) of $\mathrm{x}[\mathrm{n}]$ is given by:

$X\left(e^{j \omega}\right)=\sum_{k=-\infty}^{\infty} x_{k} \cdot e^{-j \omega k}$

where $\omega$ is the angular frequency and $\mathrm{k}$ is the kth sample. DTFT is complex in general, even for real signals. 
To plot DTFT, the magnitude and phase are plotted separately. Alternatively, the real and the imaginary parts can be plotted. The return signal from the DTFT can be computed and is known as Inverse DTFT (IDTFT), given by the following equation:

$x_{n}=\frac{1}{2 \pi} \int_{-\pi}^{\pi} X\left(e^{j \omega}\right) \cdot e^{j \omega n} d \omega$

As a function of $\omega$, the DTFT is periodic over period $2 \pi$. It is important to note that the definition of DTFT may not converge.

Some properties of DTFT are:

Linearity: If $x[n] \leftrightarrow X\left(e^{j \omega}\right)$ and

$$
\begin{gathered}
y[n] \leftrightarrow Y\left(e^{j \omega}\right), \text { then } \\
a \cdot x[n]+b \cdot y[n] \leftrightarrow a \cdot X\left(e^{j \omega}\right)+b \cdot Y\left(e^{j \omega}\right) .
\end{gathered}
$$

Convolution: If $z[n]=x[n] * y[n]=\sum_{k=-\infty}^{\infty} x[k] \cdot y[k-n]$, then

$$
\begin{gathered}
z[n] \leftrightarrow X\left(e^{j \omega}\right) \cdot Y\left(e^{j \omega}\right) . \\
z[n]=x[n] * y[n]=X\left(e^{j \omega}\right) \cdot Y\left(e^{j \omega}\right) .
\end{gathered}
$$

Modulation: If $z[n]=x[n] \cdot y[n]$, then

$$
x[n] \cdot y[n] \leftrightarrow 1 / 2 \pi \int_{0}^{2 \pi} X\left(e^{j \theta}\right) \cdot Y\left(e^{j(\omega-\theta)}\right) \cdot d \theta=Z\left(e^{j \omega}\right)
$$

The Discrete Fourier Transform (DFT) is a sampled version of DTFT. DTFT is periodic in $[0-2 \pi]$ period $2 \pi$. DTFT is sampled M times, equally spaced in frequency. DFT is a sampled version of DFTF in the frequency domain.

The sampled signal becomes our M point DFT, which is also discrete in frequency. DTFT was taken off a discrete signal, and it was in itself in continuous frequency. The 
time domain signal was in discrete time, but the frequency DTFT is in continuous frequency. The DFT is taken off a discrete time signal, and the DFT itself is in discrete frequency. The transform has M number of components [1].

The M-length DFT of $\mathrm{x}[\mathrm{n}]$ is defined as samples of the DTFT and is given by:

$$
X_{k}=\frac{1}{\sqrt{M}} \cdot X\left(e^{\frac{j 2 \pi \cdot k}{M}}\right)=\frac{1}{\sqrt{M}} \cdot \sum_{i=-\infty}^{\infty} x_{k} \cdot e^{\frac{-j 2 \pi \cdot k}{M}}
$$

When and how can $\mathrm{x}[\mathrm{n}]$ be recovered from $X_{k}$ ? When the length of the signal $\mathrm{x}[\mathrm{n}]$ is not more than $M$, i.e., when $x[n]=0$ for $n<0$ and $n>M-1$, then:

$$
x[n]=\frac{1}{\sqrt{M}} \sum_{i=0}^{M-1} X_{i} e^{\frac{j 2 \pi \cdot n_{i}}{M}}
$$

From now on, it is assumed that $x[n]=0$ for $n<0$ and $n>M-1$. This is the condition set in order to calculate the inverse Fourier transform.

DFT can also be written in the following way:

Define $x=(x[0], x[1], \ldots, x[M-1]) \quad$ as $\quad$ a discrete time domain signal, and $X=\left(X_{0}, X_{1}, \ldots . X_{M-1}\right)$ as a discrete frequency domain signal. They are both vectors.

The equivalent definition of DFT is given by the following equation:

$X=x \cdot D$

$D$ is a matrix given by:

$$
D=\frac{1}{\sqrt{M}}\left(\begin{array}{ccccccc}
1 & 1 & 1 & . & \cdot & \cdot & 1 \\
1 & e^{-j \frac{2 \pi}{M}} & e^{-j \frac{4 \pi}{M}} & . & \cdot & \cdot & e^{-j \frac{2(M-1) \pi}{M}} \\
\cdot & . \dot{2(M-1) k} & e^{-j \frac{k(M-1) \pi}{M}} & . & \cdot & \cdot & . \\
1 & e^{-j \frac{2 \pi(M-1)^{2}}{M}}
\end{array}\right)
$$


$\mathrm{x}[\mathrm{n}]$ has $\mathrm{M}$ components, so it can be expressed as a vector. $\mathrm{X}$ also has $\mathrm{M}$ components, and it is also a vector.

The vector $\mathrm{X}$ can be expressed in terms of $\mathrm{x}$ by:

$x=X \cdot D^{-1}$

$D^{-1}$ is the inverse matrix $\mathrm{D}$, given by:

$$
D^{-1}=\frac{1}{\sqrt{M}}\left(\begin{array}{ccccccc}
1 & 1 & 1 & . & . & . & 1 \\
1 & e^{j \frac{2 \pi}{M}} & e^{j \frac{4 \pi}{M}} & . & . & . & e^{j \frac{2(M-1) \pi}{M}} \\
\cdot & e^{j \frac{(\dot{M}-1) k}{M}} & e^{j \frac{k(\dot{M}-1) \pi}{M}} & . & . & . & . \\
1 & . \cdot & e^{j \frac{2 \pi(M-1)^{2}}{M}}
\end{array}\right)
$$

The properties of DFT and IDFT are quite similar to the properties of DTFT and IDTFT because DFT and IDFT are the sampled versions.

Properties of DFT include:

Linearity: $D F T(a \cdot x+b \cdot Y)=a \cdot \operatorname{DFT}(x)+b \cdot \operatorname{DFT}(y)$

Convolution: The cyclic convolution of $\mathrm{x}$ and $\mathrm{y}$ is defined as the vector/sequence $\mathrm{z}$ :

$z_{k}=\sum_{i=0}^{M-1} x_{i} \cdot y_{k-j}$, where $(k-i)$ is taken modulo M.

The cyclic convolution of $\mathrm{x}$ and $\mathrm{y}$ is denoted by:

$x \otimes_{c} y$

$\operatorname{DFT}\left(x \otimes_{c} y\right)=Z$

$Z_{i}=X_{i} \cdot Y_{i}$

Point wise multiplication:

If $\mathrm{z}$ is such that $z_{i}=x_{i} \cdot y_{i}$ for each $\mathrm{i}$, then $\operatorname{DFT}(z)=X \otimes_{c} Y$

Cyclic shift: 
If $\mathrm{y}$ is the cyclic shift of $y_{i}=x_{i-1}(\mathrm{y}$ is shifted with respect to $\mathrm{x}$ ) and $(i-1)$ is taken modulo $\mathrm{M}$ for all $\mathrm{i}$, then:

$Y_{k}=e^{-j \frac{2 \pi k}{M}} \cdot X_{k}$, DFT of $\mathrm{y}$ has a relation with DFT of $\mathrm{x}$.

Linear convolution:

Graphically, convolution can be viewed as a flip, shift, multiply and add (see Figure 4.2).

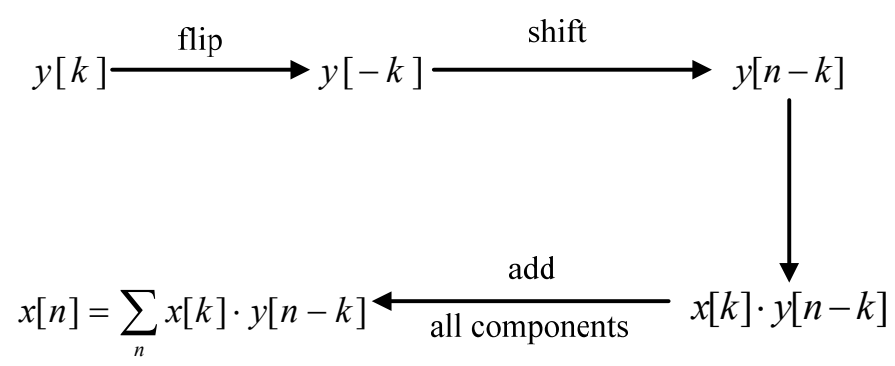

Fig. 4.2 Convolution

length $(x[n] * y[n])=$ length $(x[n])+$ length $(y[n])-1$

In the case of cyclic convolution, the same principle can be applied except that the shifting and flipping operations are completed cyclically. If any component exceeds $\mathrm{M}$, it is brought back inside. If it reaches $\mathrm{M}$, it is returned to zero, and if it reaches $\mathrm{M}+1$, it is returned to one, and always returned to the range of $0-(M-1)$ (see Figure 4.3).

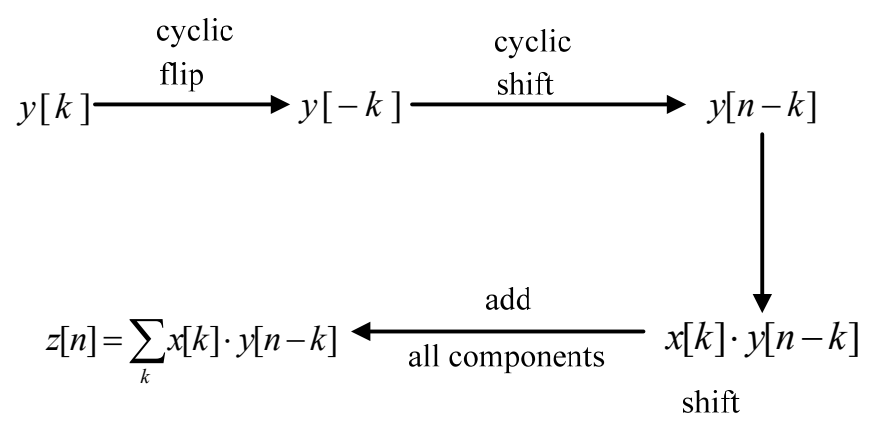

Fig. 4.3 Cyclic Convolution 


\subsection{OFDM}

Single carrier modulation presents two main problems when used in frequency selective channels. These two problems are [2]: (1) frequency selective channels introduce inter symbol interference at the receiver; and (2) equalization at the receiver may also amplify noise in frequencies where channel response is poor. As a result, single carrier modulation is affected due to high attenuations in some bands. Since the same carrier uses the entire bandwidth, this problem can become very serious (see Figure 4.4 below).

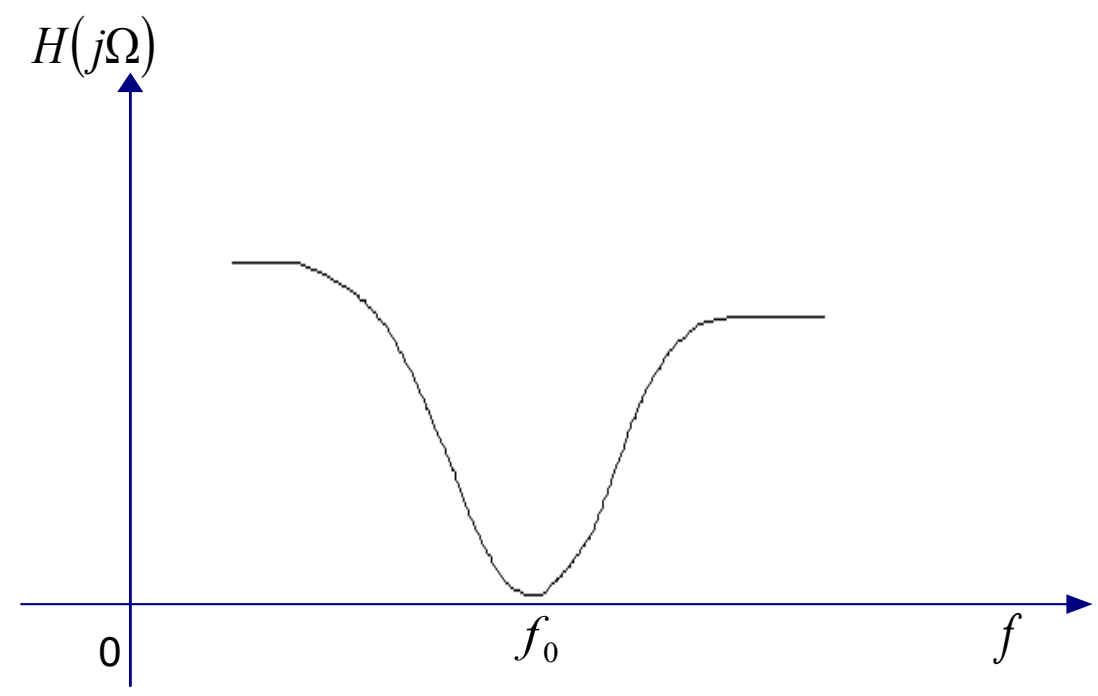

Fig. 4.4 Channel Response

The bandwidth must be divided into many small bands, and then a carrier may be allocated in each one. Furthermore, the data stream should be divided into many parallel data streams, modulating individual carriers. Then, the signals can be added together and transmitted. Thus, the entire bandwidth will be used, but with many individual and smaller carriers (see Figure 4.5). By using OFDM, the channel can be employed, thus transmitting very little information in the band. In others bands not so severely affected, 
transmission can occur at higher rates [42]. If each sub-carrier is narrow enough, then the channel frequency response is almost flat in that particular sub-band. This is true even in the strongly affected channel.

Some advantages of OFDM are as follows: (1) the available spectrum is divided into smaller sub-bands; (2) data is divided in the transmitter site, and each sub-stream modulates one sub-carrier; (3) power and rate of transmission in a band depend on the channel response on that band; and (4) no ISI, since in each narrow sub-band, the channel response is almost flat.

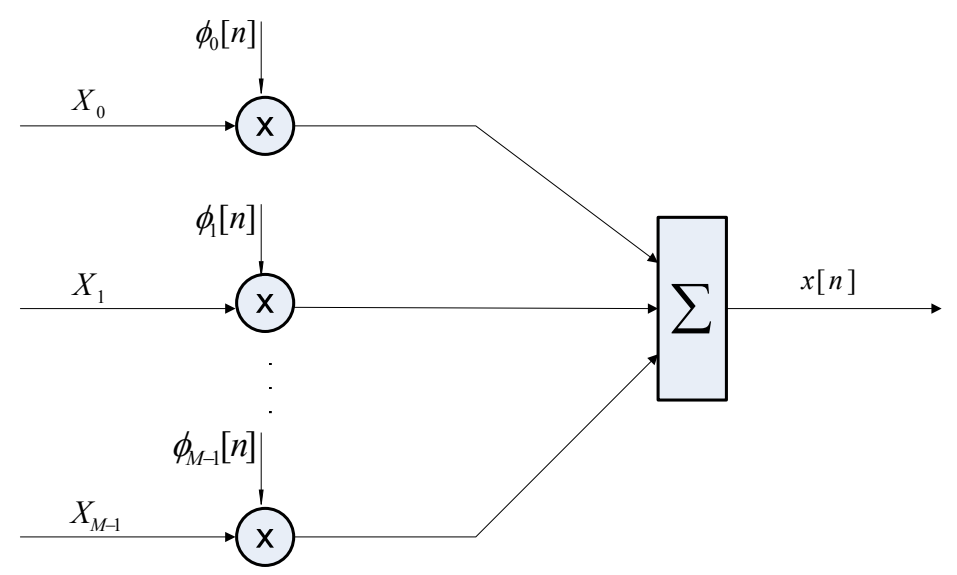

Fig. 4.5 OFDM Principle of Operation

The discussion now moves to a discrete time situation. The transmitted signal, channel, and received signal are in discrete time. In any symbol interval, $\mathrm{M}$ symbols $X_{0}, X_{1}, \ldots . X_{M-1}$ will be transmitted using M carriers $\phi_{0}[n], \phi_{1}[n], \ldots . \phi_{M-1}[n]$. The resulting signal is given by:

$x[n]=X_{0} \phi_{0}[n]+X_{1} \phi_{1}[n]+\cdots \cdots X_{M-1} \phi_{M-1}[n]$

The carriers will be at different frequencies. 
Ideally the carriers should have a brick-walled spectrum, so there is no inter symbol interference (ISI) (see Figure 4.6). This is not practical because it requires infinite length carriers in the time domain. The signal in the time domain would be a sinc function. While a different kind of spectrum is needed, ISI must also be avoided [48].

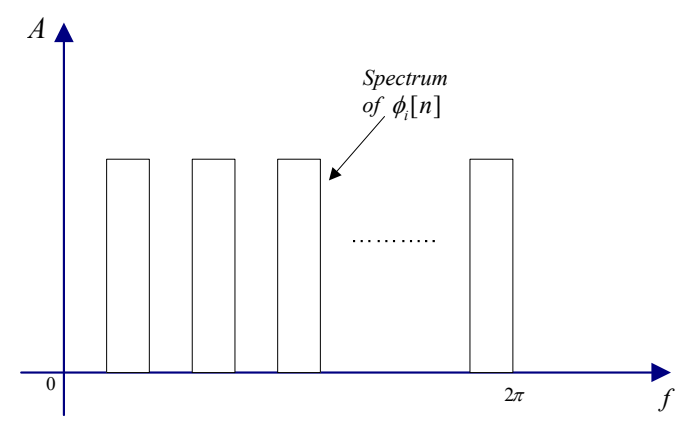

Fig. 4.6 Ideal Pulse Shape

Thus, $\phi_{i}[n]$ is selected to be orthogonal, so although they overlap with each other, there is no ISI. The IDFT carriers can be orthogonal, so they are selected as carriers. We take $\mathrm{M}$ different rows of the IDFT matrix $D^{-1}$ as $\mathrm{M}$ sub-carriers. Rows of the IDFT matrix should be considered carriers:

$$
\phi_{i}[n]=\frac{1}{\sqrt{M}} e^{j \frac{2 \pi \cdot n_{i}}{M}} i t h \text { row of the IDFT matrix. } \phi_{i}[n] \text { in the frequency domain has a }
$$

spectrum, as shown in Figure 4.7

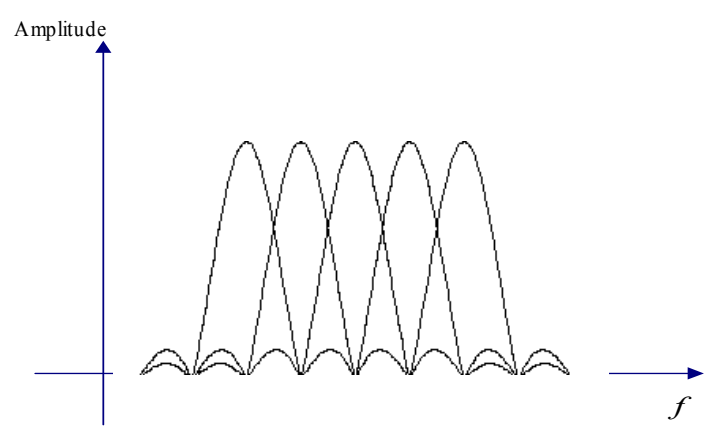

Fig. 4.7 Orthogonal Carriers 
The transmitter will be implemented as:

$x[n]=X_{0} \phi_{0}[n]+X_{1} \phi_{1}[n]+\cdots \cdots X_{M-1} \phi_{M-1}[n]=\frac{1}{\sqrt{M}} \sum_{i=0}^{M-1} X_{i} e^{j \frac{2 \pi \cdot n_{i}}{M}}=\operatorname{IDFT}(x)$

It is simply the IDFT of the vector $x$.

IDFT can be implemented using the Fast Fourier Transform, which is a very efficient algorithm used to calculate IDFT. A block diagram of the transmitted signal is depicted in Figure 4.8 below.

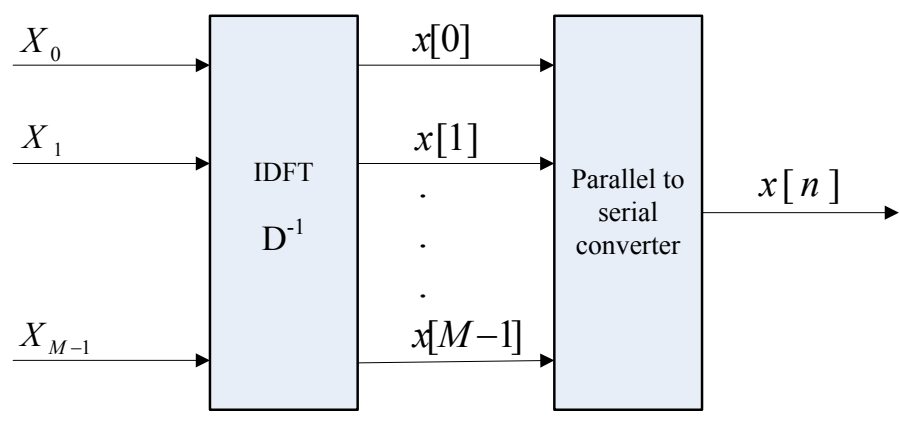

Fig. 4.8 IDFT Implementation

When the blocks formed by $x[n]$ are transmitted, the blocks are formed by a set of symbols. The channel causes inter block interference (IBI). The transmitted blocks have the structure indicated in Figure 4.9.

Transmitted

Signal

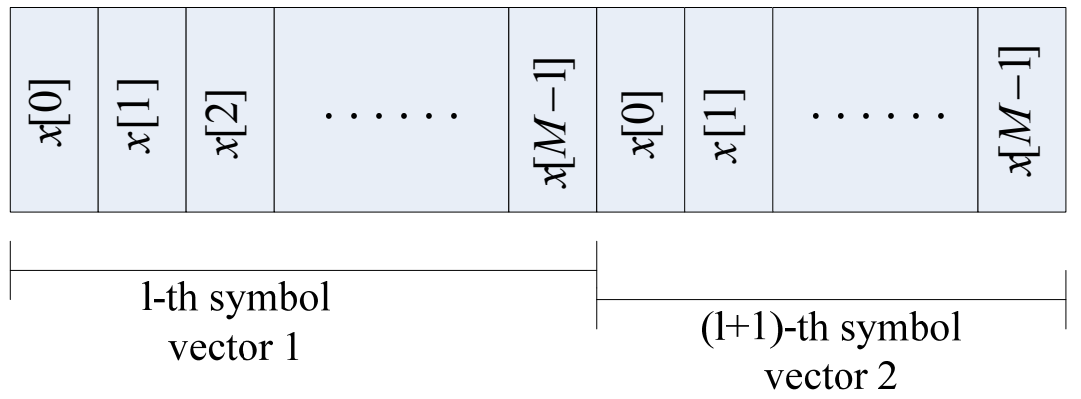

Fig. 4.9 OFDM Blocks at Transmitter 
The linear convolution of the vector $x[n]$ with the channel impulse response will have a length of $M+(L-1)$. $\mathrm{L}$ is the channel impulse response length. Those L-1 symbols will interfere with the next block, the first samples of the next block, producing IBI.

At the receiver site, the blocks are received with the structure shown in Figure 4.10. By adding extra zeros after every block, IBI can be controlled, and the block structure is depicted in Figure 4.11. After convolution, only the extra zeros will be affected, and the second block is preserved [45]. The signal does not have ISI, but the transmission rate is compromised.

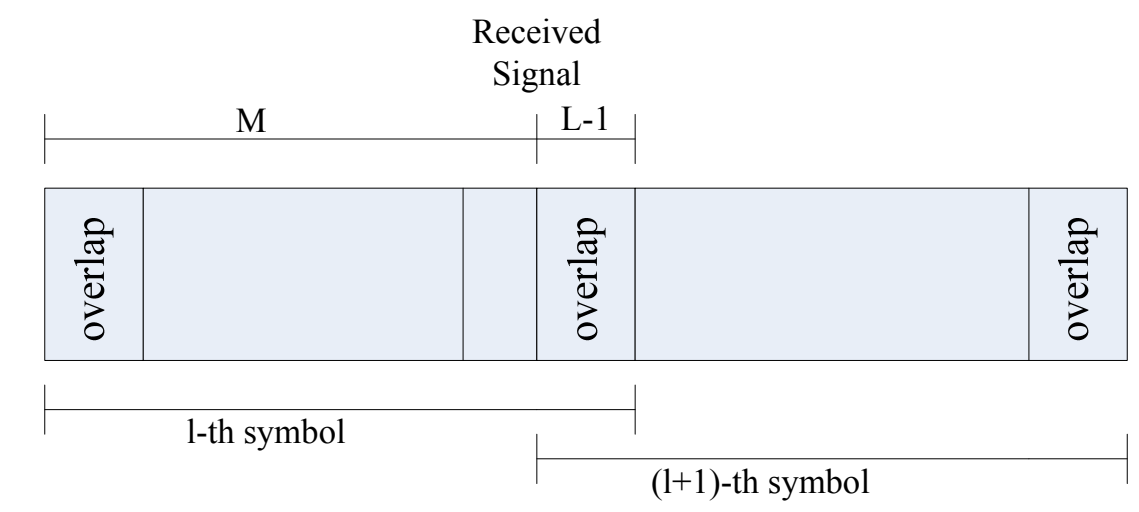

Fig. 4.10 OFDM Blocks at Receiver

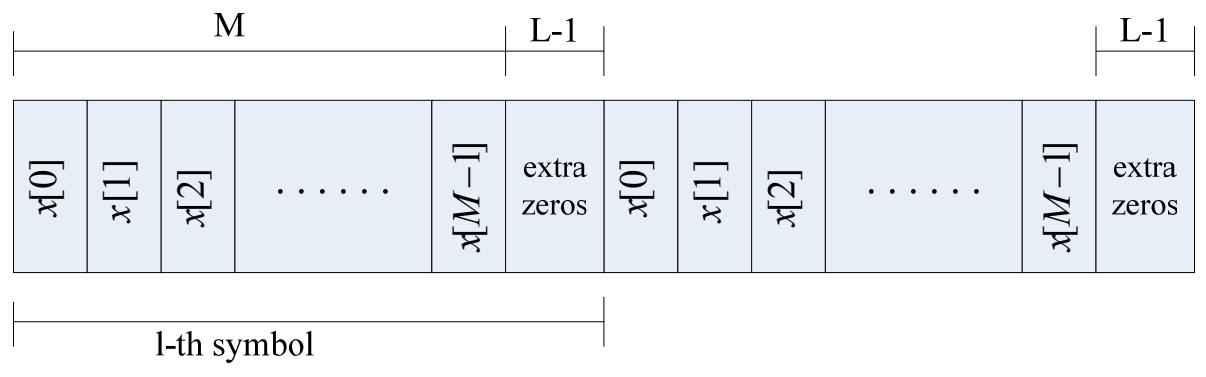

Fig. 4.11 OFDM Blocks with Extra Zeros added 
Another way to avoid ISI is to use a cyclic prefix [48]. The L-1 samples are copied at the beginning of every block. At the transmitter, IDFT is performed, and at the receiver, the DFT of the received block is performed (see below).

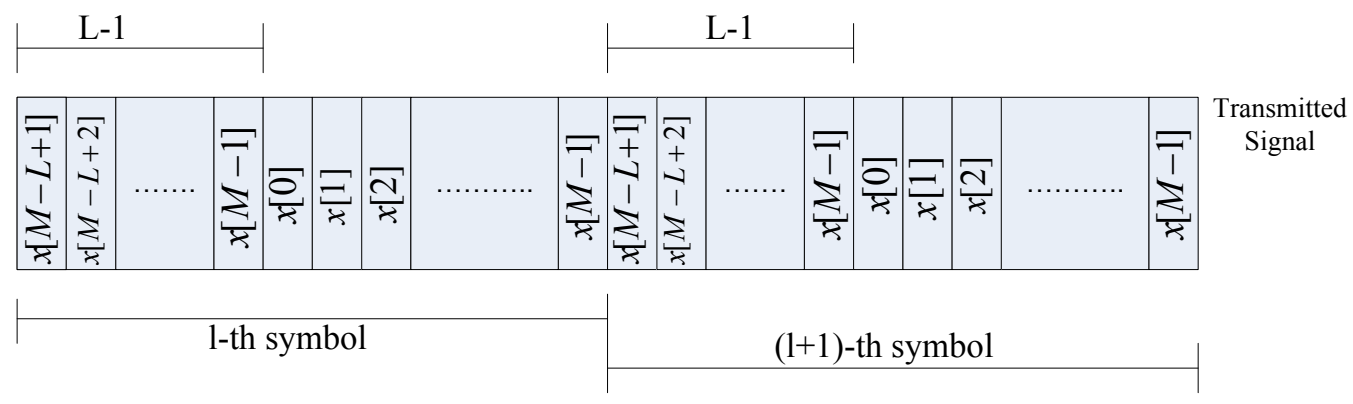

Fig. 4.12 OFDM Received Blocks with No Overlapping

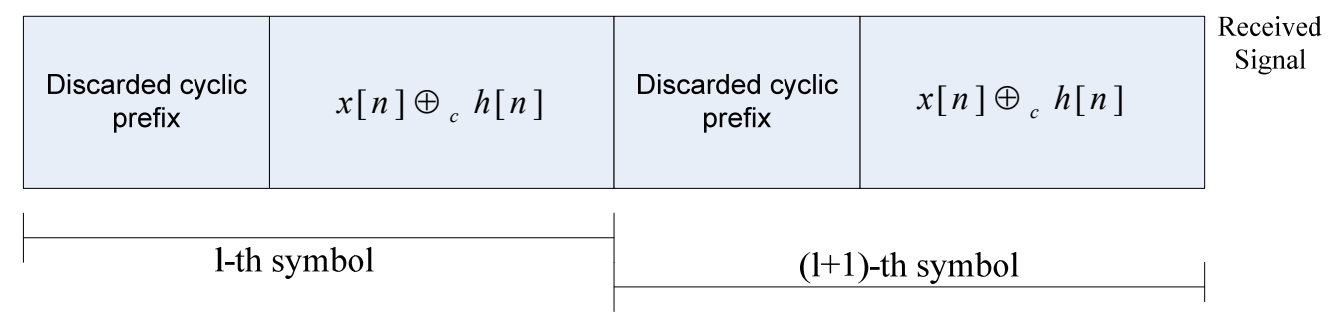

Fig. 4.13 Received Signal as the Result of Cyclic Convolution

$y[n]=x[n] \otimes_{c} h[n]$ is the cyclic convolution of the transmitted signal $x[n]$ and the channel impulse response $h[n]$. The lth block will be linearly convoluted with the channel impulse response. As mentioned before, at the receiver, the DFT of the received block is performed; by the properties of convolution, the DFT of $x[n]$ times the DFT of $h[n]$ can be determined. Convolution in time domain $y[n]=x[n] \otimes_{c} h[n]$ is the multiplication in the frequency domain. Figure 4.14 shows a block diagram of an OFDM communication system. 


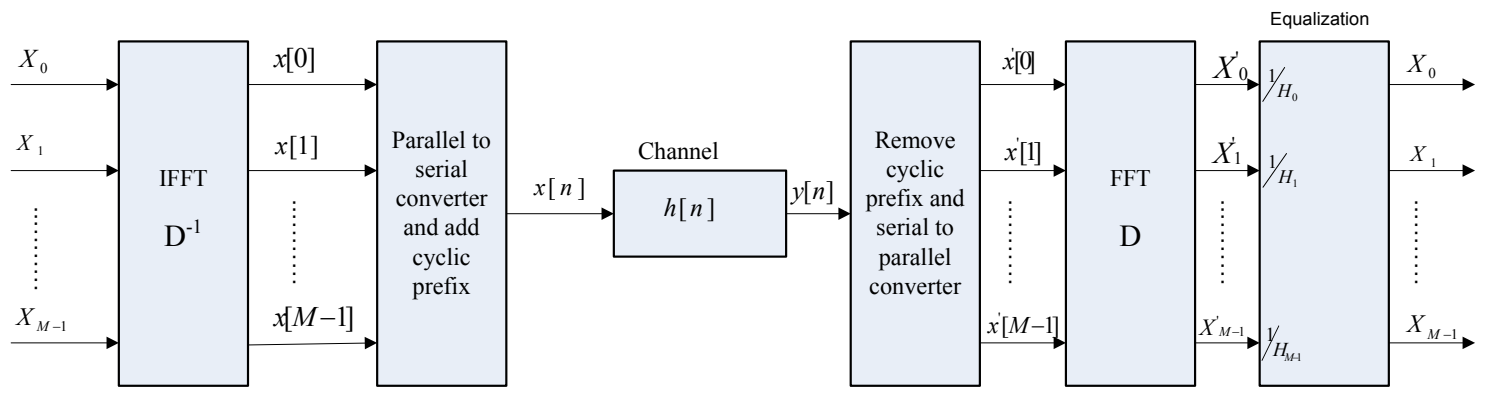

Fig. 4.14 OFDM Transmitter and Receiver

Before the equalization process:

$\operatorname{DFT}(x[n]) \cdot \operatorname{DFT}(h[n])$ or $x[n] \otimes_{c} h[n]$

$\left(H_{0}, H_{1}, \ldots, H_{M-1}\right)=\operatorname{DFT}(h[n])$

The resulting signal is then divided by the channel coefficients to obtain the original data $x[n]$. Thus, it can be observed how the properties of DFT are used, especially the properties of cyclic convolution.

If the channel is poor, i.e., $H_{i}$ is very low, the noise component in branch $H_{i}$ will be multiplied by a high number. The noise component added to $X_{i}$ will be large, and $X_{i}$ will have low SNR. Depending on the magnitude of the $\left(H_{0}, H_{1}, \ldots, H_{M-1}\right)$ components, some sub-bands will be better than others.

The good sub-channels will be used to a greater extent than the sub-channels that are poor. The noise variance in the ith sub-channel is $\delta^{2} /\left|H_{i}\right|^{2}$, where $\delta^{2}$ is the received noise variance (see Figure 4.15). 


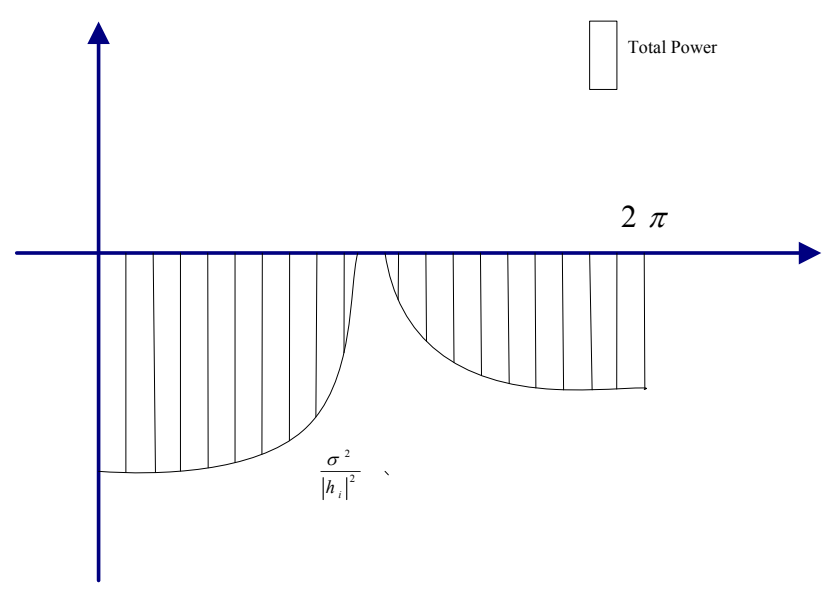

Fig. 4.15 Power Allocation for Different Sub-carriers

The power required in each sub-channel is distributed, depending on the value of $H_{i}$. Then, the number of bits to be transmitted to each sub-channel is determined. The number of bits and the constellation can be chosen for a sub-channel based on the SNR in that particular sub-channel and the required probability of error.

The next section focuses on time diversity as a way to improve the diversity gain in channels with long delays. The satellite channel is an example of channels in which time diversity can improve the performance of OFDM, so it can be used to transmit high data rates with high bandwidth efficiency.

\subsection{Time Diversity}

When the channel coherence time equals or exceeds several transmitted symbol period, indicating two symbols transmitted with a separation in time longer than the channel coherence time, the two symbols will experience channel realizations that are highly uncorrelated and can be used to obtain diversity [43]. When the signal bandwidth is much larger than the channel coherence bandwidth, the channel is frequency selective. 
The best way to achieve diversity is to form the two transmitted symbols using a repetition coding scheme. Furthermore, to guarantee that the repeated symbols will be transmitted over uncorrelated channel realizations, an appropriated interleaver will need to be applied to the stream of symbols to be transmitted.

At the receiver, the copies of the symbols must be combined together using a MRC (maximum rate combiner) in order to maximize the SNR. The SNR at the MRC output equals the sum of the SNR of the branches at the MRC input.

Using BPSK modulation and M copies of the transmitted symbol being combined with path channel gains, $H_{1}, H_{2}, \ldots . H_{R}$, the error probability that can be obtained using MRC combiner is [2]:

$Q\left(\sqrt{\frac{2}{N_{0}} \sum_{n=1}^{R}\left|h_{n}\right|^{2}}\right)$

The average probability of symbol error is:

$$
\begin{aligned}
& P_{S E R}=\int_{0}^{\infty} Q\left(\sqrt{2 \cdot S N R_{M R C}}\right) \cdot f\left(S N R_{M R C}\right) \cdot d S N R_{M R C} \\
& =\left(\frac{\left.1-\sqrt{\frac{\overline{S N R_{M R C}}}{1+\overline{S N R_{M R C}}}}\right)^{R}}{2} \sum_{m=0}^{R-1}\left(\begin{array}{c}
R+m-1 \\
m
\end{array}\right)\left(\begin{array}{c}
1+\sqrt{\frac{\overline{S N R_{M R C}}}{1+\overline{S N R_{M R C}}}} \\
2
\end{array}\right)\right.
\end{aligned}
$$

The SNR at the MRC output is given by:

$S N R_{M R C}=\left(\frac{1}{N_{0}}\right) \cdot \sum_{m=1}^{R}\left|h_{n}\right|^{2}$

This can be reduced using upper bounds to: 


$$
P_{S E R} \leq \prod_{m=1}^{R} \frac{1}{1+\frac{\overline{S N R_{M R C}}}{2}}
$$

At large SNR, this upper bound becomes:

$P_{S E R}=\left(\overline{\frac{S N R_{M R C}}{2}}\right)^{-R}$

In log form:

$\log \left(P_{S E R}\right)=-R \cdot \log \left(\overline{S N R_{M R C}}\right)$

Now, for any diversity technique, the performance improvement is manifested by the communication error probability decreasing at a high channel SNR at a much larger rate than systems with less or no diversity. When using log scales, this rate of decrease in the communication error probability becomes the slope of the line representing the communication error probability at high SNR, and this is known at the diversity gain. Diversity gain is defined as follows:

$m=-\lim _{S N R \rightarrow \infty} \frac{\log P_{S E R}}{\log S N R}$

This definition establishes an implicit behavior at high SNR for the probability of symbol error as a linear function of the SNR, when seen in a plot with log scales. It can be observed that in these conditions, the diversity gain is the slope of the linear relation. It is better to have a large diversity gain, meaning that the symbol error probability will be reduced at a faster rate.

Comparing the definition of diversity gain and the probability of symbol error at high SNR, it becomes apparent that:

$m=-R$ 
$R$ is the number of repetitions of the symbol to be transmitted.

Time diversity systems with repetition coding achieve full diversity gain [47]. However, the use of repetition coding sacrifices the total data rate. If OFDM is to be used over satellite channels, diversity gain must be guaranteed. By exploiting time diversity, OFDM can be used in satellite links, as long as the transmission of two consecutive symbols will take place in a time interval longer than the satellite coherence time.

Satellite channels present some other problems, [41] including: (1) non-linear distortion due to onboard power amplifiers; (2) long round trips; and (3) reduced time diversity. Satellite links have long uplink and downlink paths, making the round trip very large [46]. This affects the accuracy of the channel estimators that are used in OFDM terrestrial links in order to maintain updated channel conditions.

Figure 4.16 shows a proposed block diagram of a system that uses time diversity as a way to obtain diversity gain. The system uses a channel coding block to add redundancy to the transmitted signal; this channel coding could employ turbo codes (TC) that have been demonstrated to be very effective. TC employs two parallel convolutional encoders.

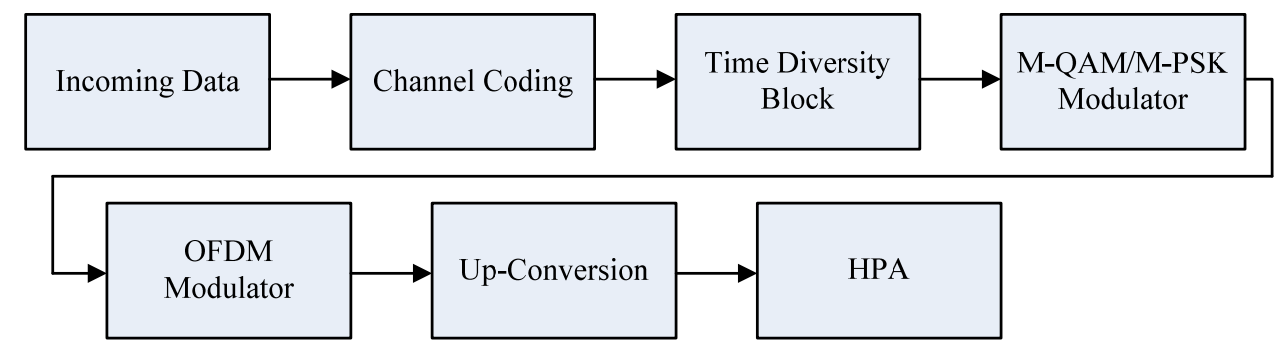

Fig. 4.16 Transmitter Implementation Using Time Diversity

3GPP Long Term Evolution (LTE) uses a similar approach in terrestrial OFDM transmission. Its physical layer lacks a time diversity scheme that can provide diversity gain. The main components in Figure 4.16 is the channel coding, mentioned in the 
previous paragraph, the time diversity block that will add the diversity gain [44], and the M-QAM or M-PSK modulator that will map incoming data into the type of constellation to be used. The modulated symbols will be arranged into a frame structure, and then allocated to the OFDM sub-carriers inside the OFDM modulator block. The signal leaving the OFDM will be up-converted for use in the satellite channel, and then amplified in the high power amplifier block. Figure 4.17 shows the time diversity block. The signal leaving the channel coding block is interleaved and interlaced.

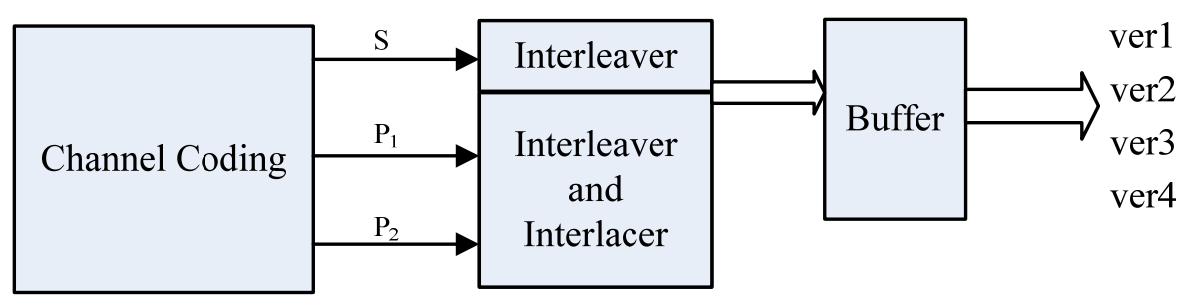

Fig. 4.17 Time Diversity Block

It is important to note that the signal from the channel coding block is formed by three streams, since the Rate $=1 / 3$. The interleaved and interlaced signals [50] are written into a buffer, in which four versions are defined. The transmitter reads a block of coded bits from the buffer. The different versions are used for different retransmissions, transmitting various parts of the coded word in distinct time instants.

The overall code rate is reduced after each transmission. For a desired code rate, the number of coded bits for transmission is calculated and then fed into the time diversity block. Puncturing and repetition can be obtained in the same operation. We then take the bits carried in the buffer and transmit them within several transmission intervals $\left(T x_{I}\right)$. 
Thus, diversity gain is obtained. LTE does not offer any time interleaving techniques other than $T x_{I}$, which is the transmission time duration of each version of the coded bits. Figure 4.18 shows the transmission of the four versions in different time allocations.

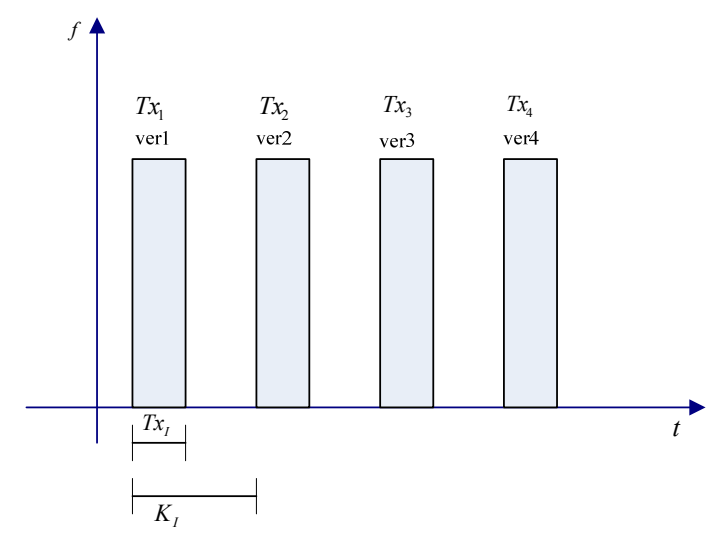

Fig. 4.18 Retransmission of Versions (ver 1, ver $2, \ldots .$, ver $N)$

We use $\mathrm{N}$ different versions $($ ver 1, ver $2, \ldots .$, ver $N)$, each of which is mapped into different $T x_{I}$, spaced by $K_{I} . K_{I}$ is a factor that determines the interleaving depths depending on the channel correlation and latency. The expression for the protected $t_{P}$ time is given by:

$t_{p}=K_{I}\left(N_{R T x}-1\right)+T x_{I}$

$N_{R T x}$ is the number of re-transmissions needed to complete the transmission of a single buffer. $N_{R T x}$ re-transmissions are used to obtain $\mathrm{N}$ versions.

The channel coherence time is calculated by:

$T_{c}=\frac{1}{K_{c}} \frac{1}{2 f_{d m}}$, where $K_{c}=1$ is a proportionality factor that depends on the threshold selected to delimit the support of the autocorrelation function, $f_{d m}$. This is the maximum Doppler shift defined by: 
$f_{d m}=\frac{v}{c} f_{0}$

We must select a value for $t_{p}$ that satisfies: $t_{p}>T_{c}$, meaning that the protection time is larger than the channel coherence time, and two consecutive symbols will be uncorrelated.

If $T_{c}=90 m s$ and we select $K_{I}=40$ and $T x_{I}=1 m s$, then:

$t_{p}=K_{I}\left(N_{R T x}-1\right)+T x_{I}=40 m s(4-1)+1 m s=121 m s$. This satisfies the condition of $t_{p}>T_{c}$. The following Table 4.1 shows some results.

Table 4.1 Time Diversity OFDM Satellite Channel

\begin{tabular}{|c|c|c|c|}
\hline$N_{R T x}$ & $K_{I}[\mathrm{~ms}]$ & $T_{c}[\mathrm{~ms}]$ & $t_{p}[\mathrm{~ms}]$ \\
\hline 4 & 40 & 90 & 121 \\
\hline 4 & 80 & 90 & 321 \\
\hline 6 & 4 & 9 & 21 \\
\hline 6 & 8 & 9 & 41 \\
\hline 12 & 16 & 9 & 177 \\
\hline
\end{tabular}

$T_{c}$ depends on the velocity of the mobile user and the central frequency $f_{0}$.

\subsection{Non-linear Distortions in Satellite Channels}

Higher order modulations, such as 8PSK and 16 QAM, are the modulation vehicles through which the higher throughputs demanded by broadcasters and satellite operators are achieved. However, this brings with it challenges that have traditionally not been 
evident with existing QPSK modulation [40]. Phase noise, higher $\mathrm{C} / \mathrm{N}$ requirements and increased dish sizes at downlink sites are several of these disadvantages. In order to address these challenges, it is necessary to develop a system solution that allows the use of higher modulation schemes over satellite channels, and at the same time can compensate for any distortions in the channel.

A satellite dynamic precorrection system will allow for the maximization of the satellite transponder throughput, significantly reducing downlink receiver antenna sizes and increasing link reliability. This dynamic precorrection will compensate for virtually any linear and non-linear distortion that is likely to be encountered in a typical satellite transmission chain. It also compensates for both earth and satellite distortions.

The effects of the satellite high power amplifiers (HPA) must also be considered when using OFDM to transmit high data rates over the satellite link. OFDM is highly sensitive to the presence of non-linear distortions and synchronization errors between transmitted and received signals.

Digital pre-correction schemes can be applied for the compensation of the AM/AM and AM/PM distortion, introduced by onboard satellite HPA. This linearization cannot be realized successfully, unless the path delay introduced by the analog chain is previously estimated.

This type of non-linear distortion is solely dependent on the modulus of the input signal and appears at the receiver as a warped symbol constellation, thereby degrading the bit error rate (BER); in the frequency domain, the distorted signal undergoes spectral re-growth, which generates intermodulation products and adjacent channel interference. 
The time delay introduced by the analog chain responsible for frequency upconversion to the HPA input and frequency down-conversion from the HPA output must be compensated for, before estimation of the pre-correction coefficients.

A time delay estimation module is necessary before any adaptive pre-correction scheme is initiated. The time delay estimation algorithm proposed in [39] is accurate and can be employed in satellite HPA. This algorithm is based on the definition of an intelligent cross-correlation between the input and output of the HPA signals.

They used a Saleh model for the travelling wave tube amplifier (TWTA), as it introduces more significant $\mathrm{AM} / \mathrm{PM}$ distortions than the solid state power amplifier (SSPA). The memory-less model of the HPA is defined by:

$A[|x|]=\frac{\alpha_{a}|x|}{1+\beta_{a}|x|^{2}}$

$\Phi[|x|]=\frac{\alpha_{\Phi}|x|^{2}}{1+\beta_{\Phi}|x|^{2}}$

$\alpha_{a}=2 \quad \beta_{a}=\beta_{\Phi}=1 \quad \alpha_{\Phi}=\frac{\pi}{3}$

The input saturation voltage $A_{s}=\frac{1}{\sqrt{\beta_{a}}}$ and the maximum output amplitude $A_{\max }=\alpha_{a} \frac{A_{s}}{2}$ are both normalized to 1 .

The phase distortion at the saturation point is $\Phi_{\text {sat }}=\Phi\left[\frac{1}{\sqrt{\beta_{a}}}\right]=\frac{\pi}{6} \mathrm{rads}$. 


\subsection{Simulation Results}

Figure 4.19 shows the simulation block diagram. For the simulation, OFDM 16QAM and OFDM QPSK modulations were used. The satellite channel is presented as a Rician model with a path loss block that simulates the signal attenuation from the satellite to the earth terminal. The satellite is of a geostationary orbit (GEO Satellite).

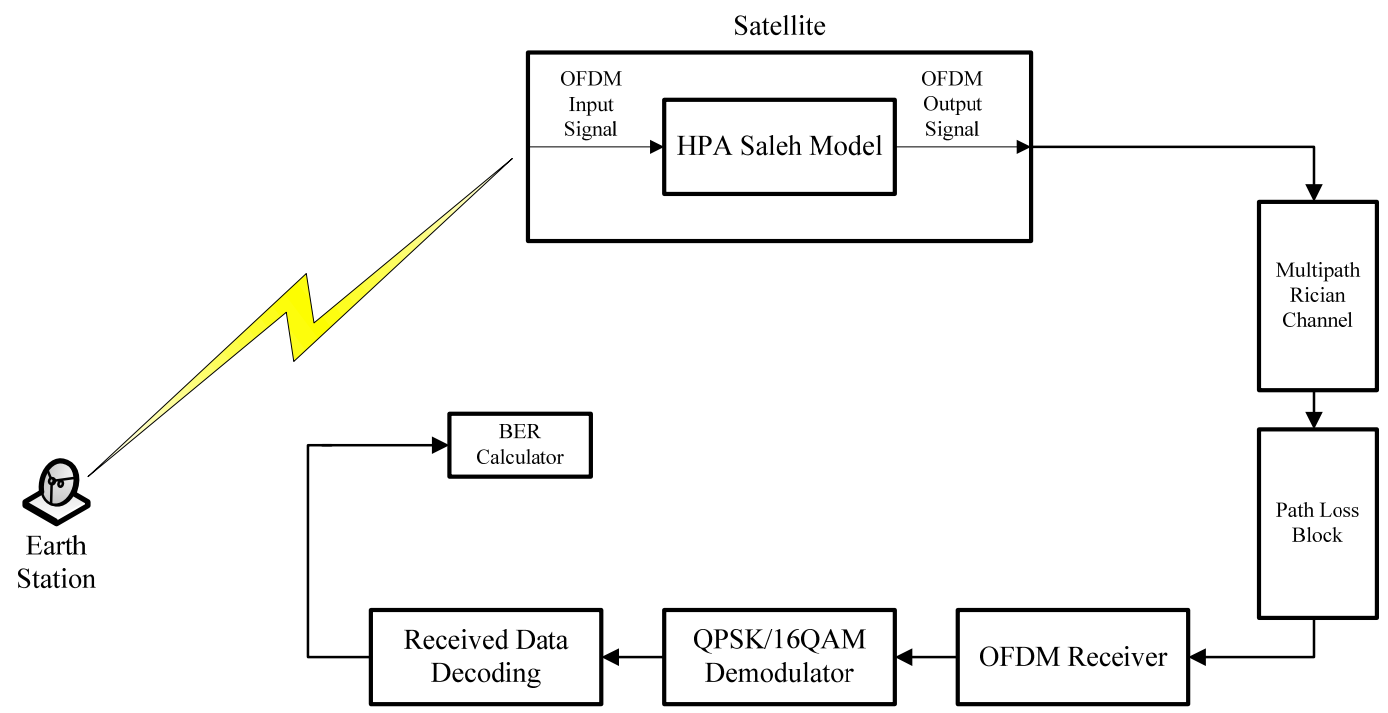

Fig. 4.19 Block Diagram of OFDM Satellite Link

The parameters of the simulation are listed as follows:

Bandwidth: $5 \mathrm{MHz}$

Central frequency: $2 \mathrm{GHz}$

OFDM sub-carrier spacing: $\Delta f=15 \mathrm{KHz}$

OFDM IFFT size: 2048 for 16QAM

OFDM IFFT size: 1024 for QPSK

$T x_{I}=1 \mathrm{~ms}$

OFDM symbol time: $83.33 \mu \mathrm{s}$

Number of OFDM symbols: 12 
Cyclic prefix duration: $16.67 \mu \mathrm{s}$

Rician factor: $\mathrm{K}=2$

Maximum Doppler shift: $15 \mathrm{~Hz}$

Satellite-earth station distance: $35000 \mathrm{Km}$

Satellite path loss: $180 \mathrm{~dB}$

Fig. 4.20 reveals the OFDM spectrum of a QPSK OFDM uplink and downlink signals, in addition to HPA Effects on the OFDM spectrum.

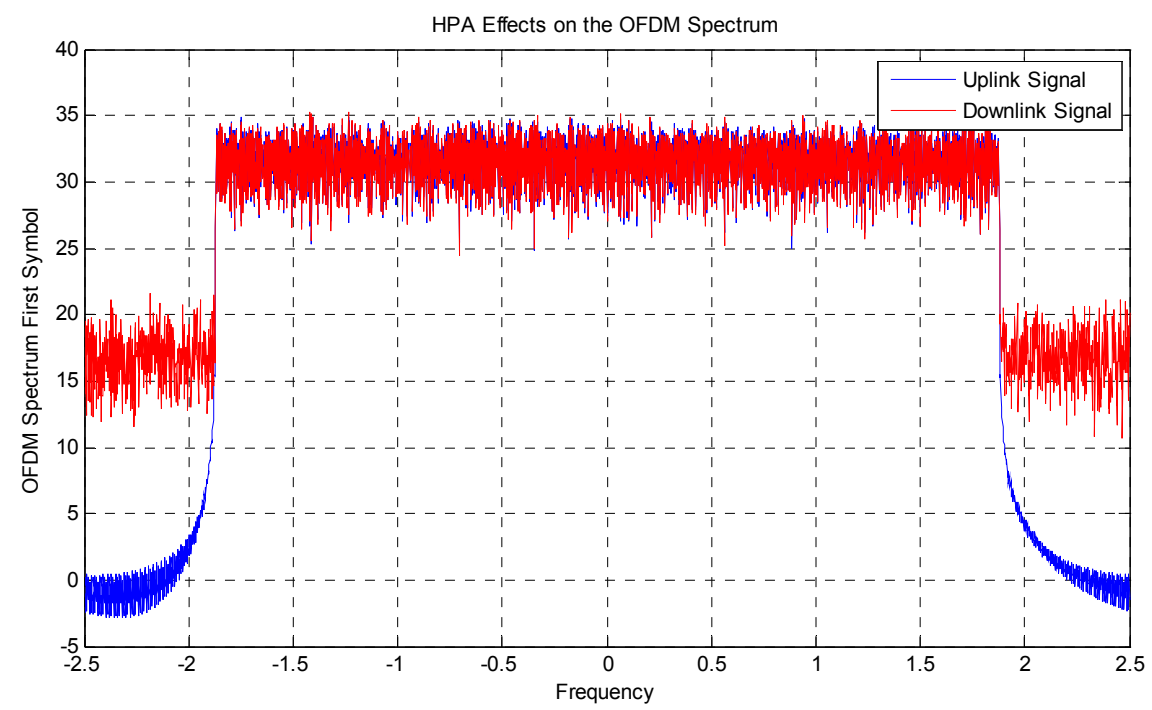

Fig. 4.20 QPSK OFDM Saturation Level = $0 \mathrm{db}$ Relative to AM Average

The downlink spectrum is severely attenuated. This can be problematic, especially for higher order modulation schemes that result in the receiver BER performing poorly. The HPA response compared to the saturation level for the values of Figure 4.20 is shown in Figure 4.21. Clearly, the HPA response is much attenuated compared to the reference linear value. Furthermore, Fig. 4.22 shows the QPSK uplink and downlink constellations. 


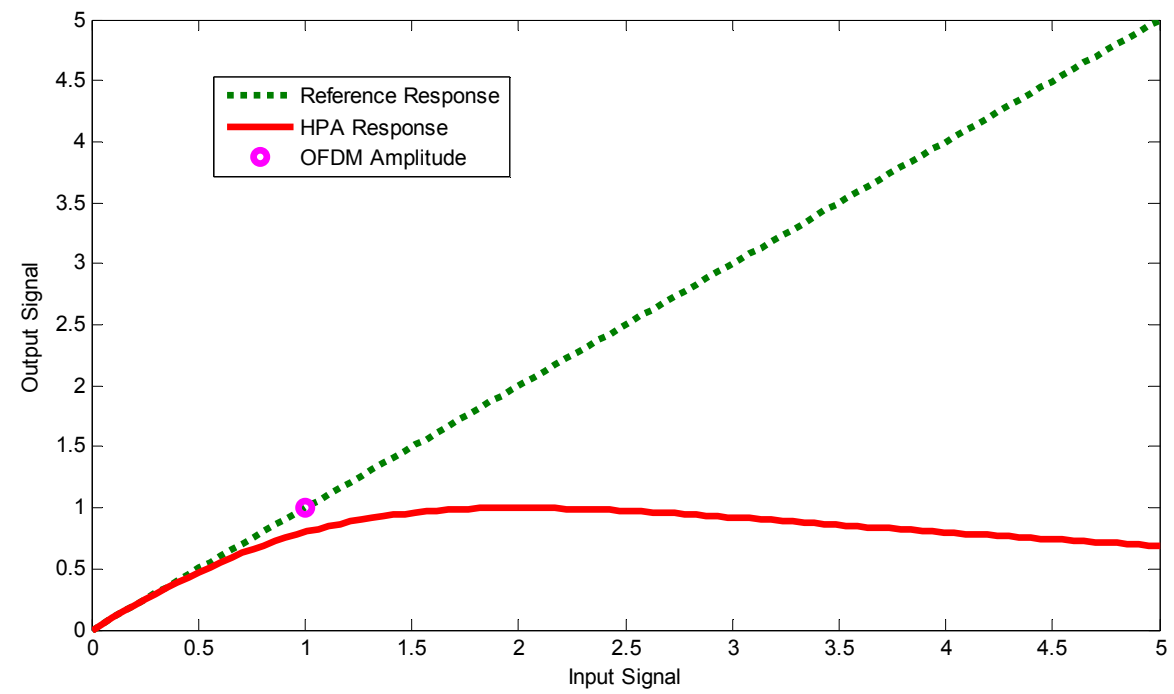

Fig. 4.21 HPA Response for QPSK with Saturation Level of $0 \mathrm{~dB}$

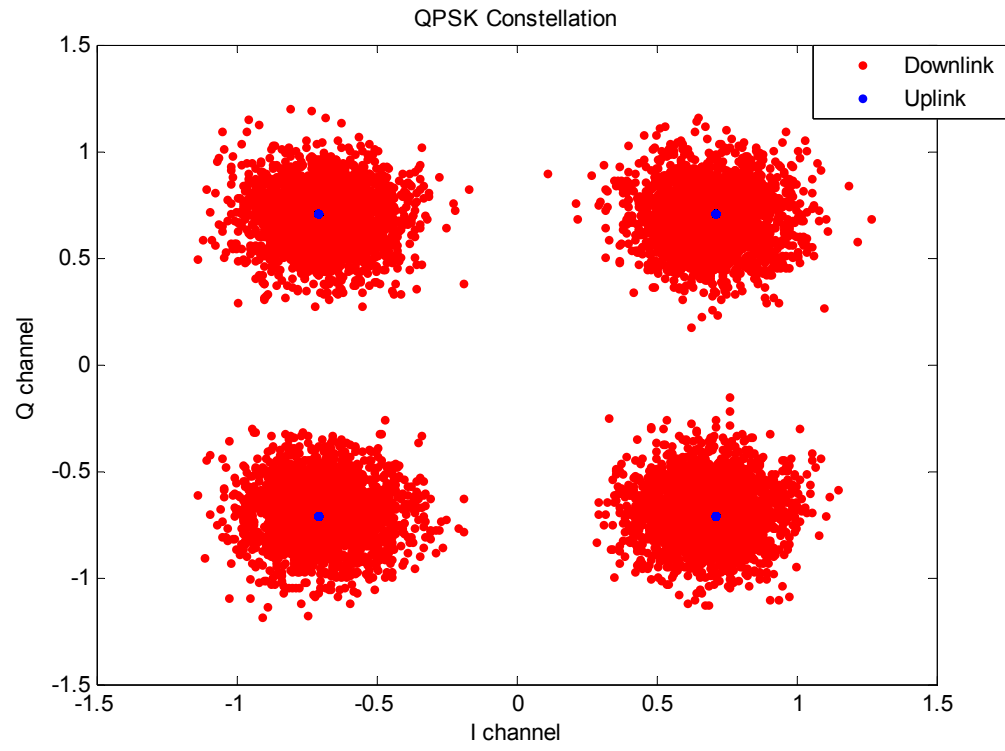

Fig. 4.22 OFDM QPSK Constellation Saturation Level $0 \mathrm{~dB}$, Path Loss $=180 \mathrm{~dB}$

Now, the saturation level is increased to $5 \mathrm{~dB}$. The effects on the QPSK OFDM spectrum, the HPA response and the QPSK constellation are indicated in Figures 4.23, 4.24 and 4.25, respectively. Note the improvement of the downlink QPSK OFDM 
spectrum, indicated in red, which is closer to the uplink signal (in blue), as well as the improvements of the constellation and the HPA response.

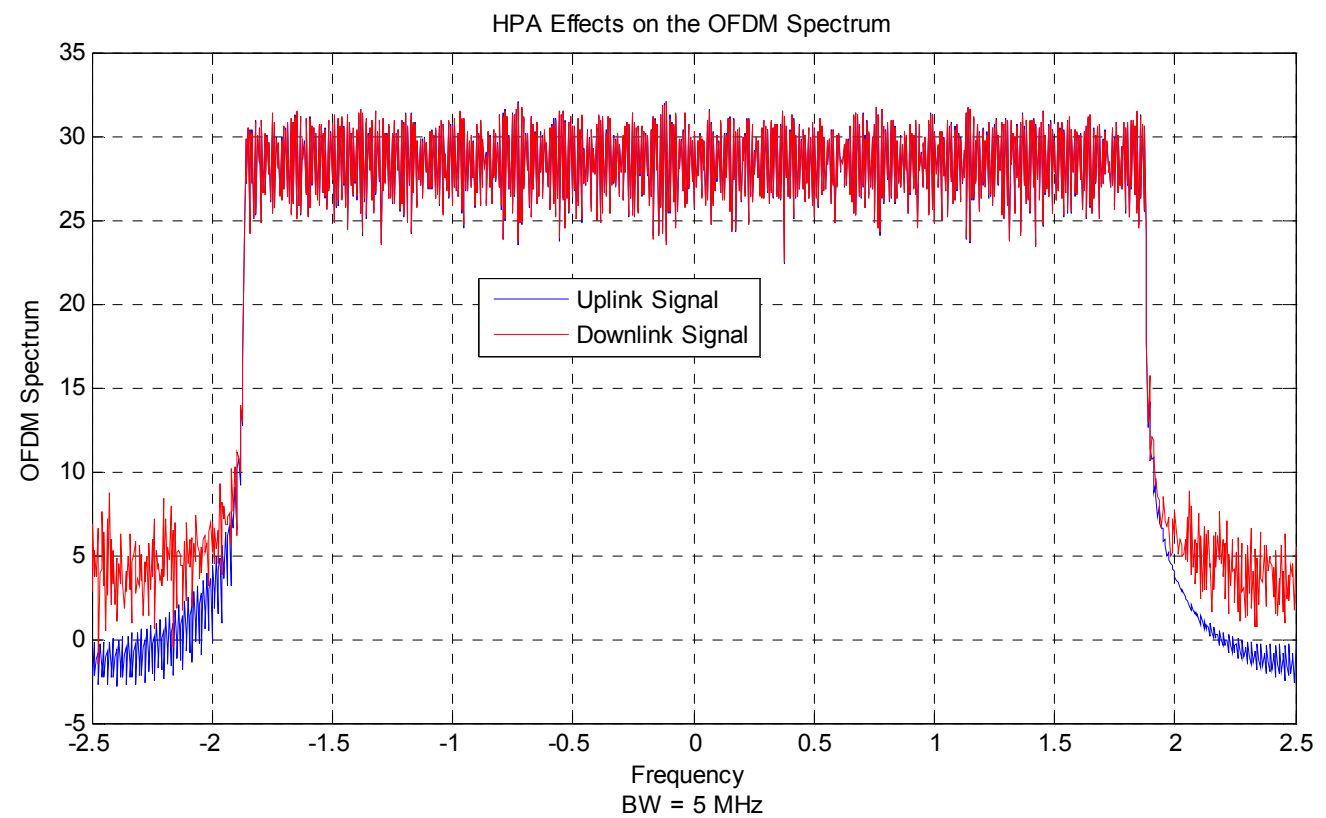

Fig. 4.23 QPSK OFDM Saturation Level $5 \mathrm{~dB}$ Relative to AM Average

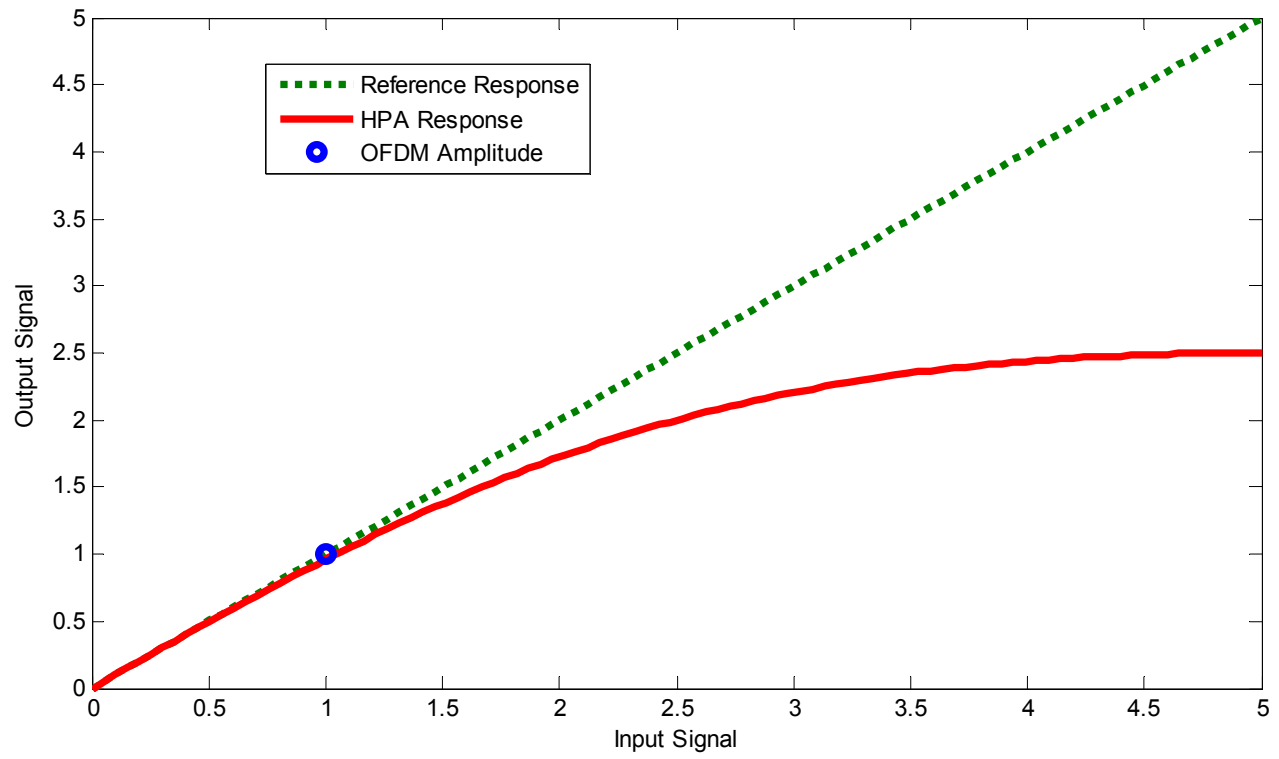

Fig. 4.24 HPA Response for QPSK with Saturation Level of $5 \mathrm{~dB}$ 
The HPA response with a saturation level of $5 \mathrm{~dB}$ becomes much closer to the linear response in the lower values of the input signal axis, making the downlink spectrum and constellation less distorted and improving the bit error rate at the receiver site.

The constellation noise, spectrum noise and attenuation are caused by the Rician channel and path loss block contributions.

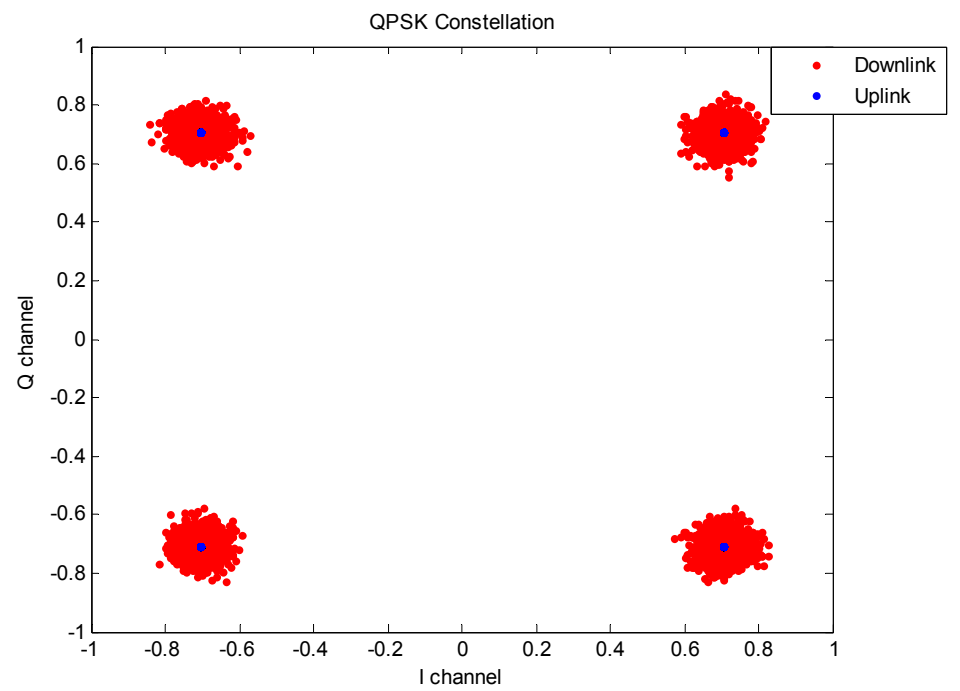

Fig. 4.25 QPSK OFDM Constellation Saturation Level $5 \mathrm{~dB}$, Path Loss $=180 \mathrm{~dB}$

Now, the same results for the case of OFDM 16QAM satellite link communication, with IFFT size of 2048, are presented below. Figures 4.26 and 4.27 show the simulation results with a saturation level of $0 \mathrm{~dB}$ and satellite channel contribution. 


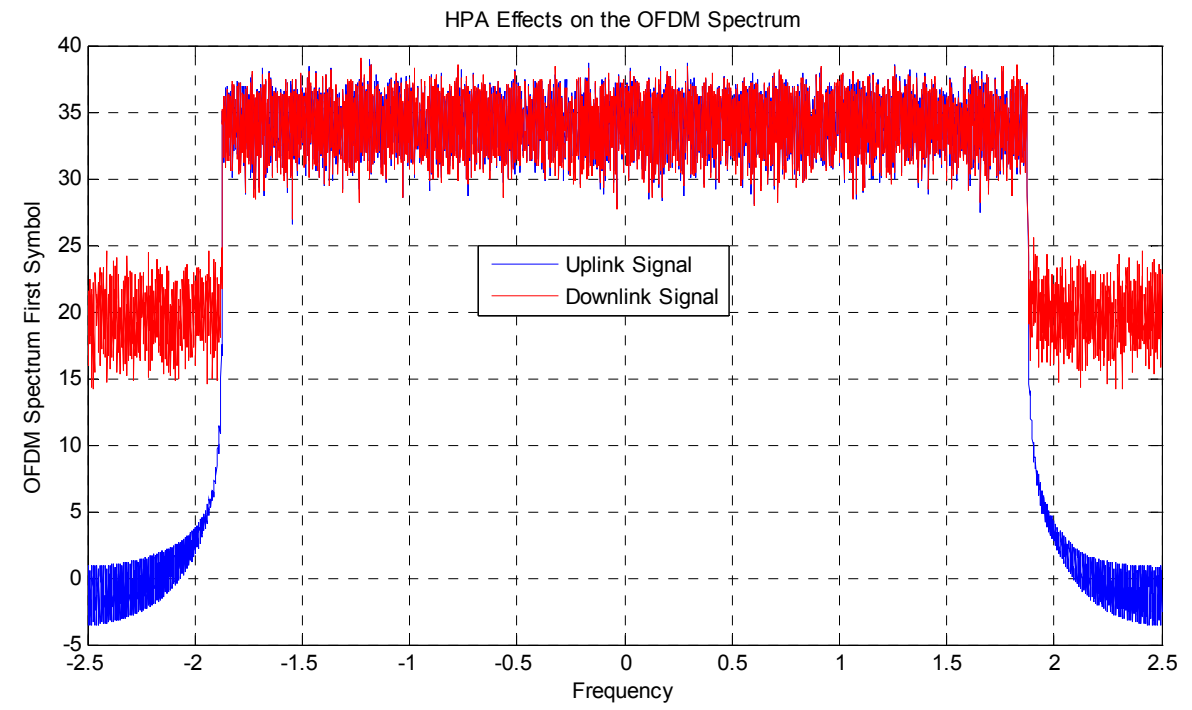

Fig. 4.26 16QAM OFDM Saturation Level $0 \mathrm{~dB}$ Relative to AM Average

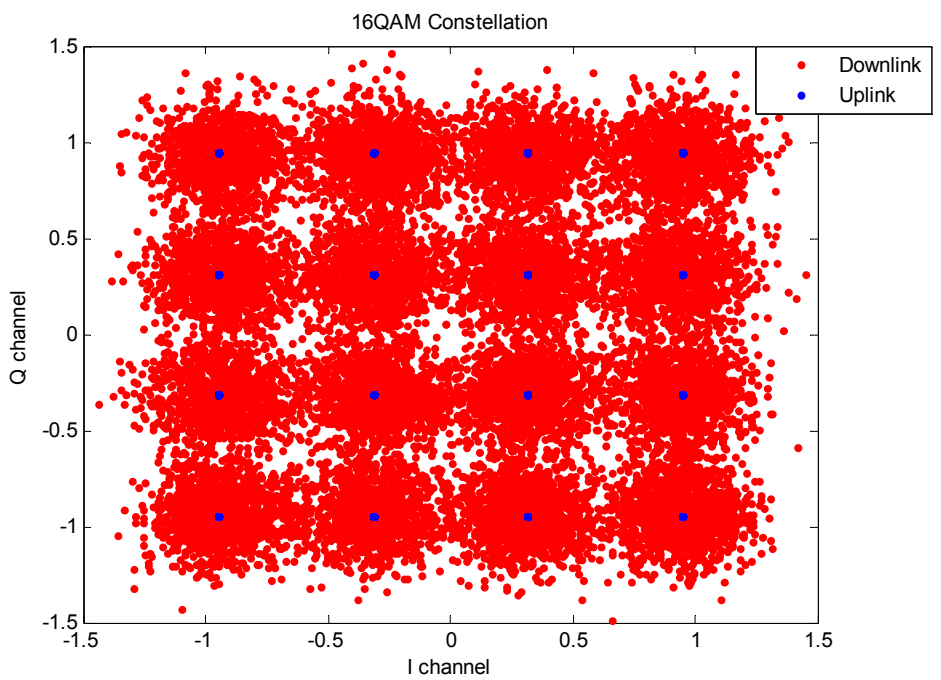

Fig. 4.27 16QAM OFDM Constellation Saturation Level 0 dB, $\mathrm{K}=2$

Next, Figures 4.28 and 4.29 show the simulation results with a saturation level of 5 $\mathrm{dB}$ and satellite channel contribution due to the multipath Rician model and the path loss block for 16QAM OFDM . 


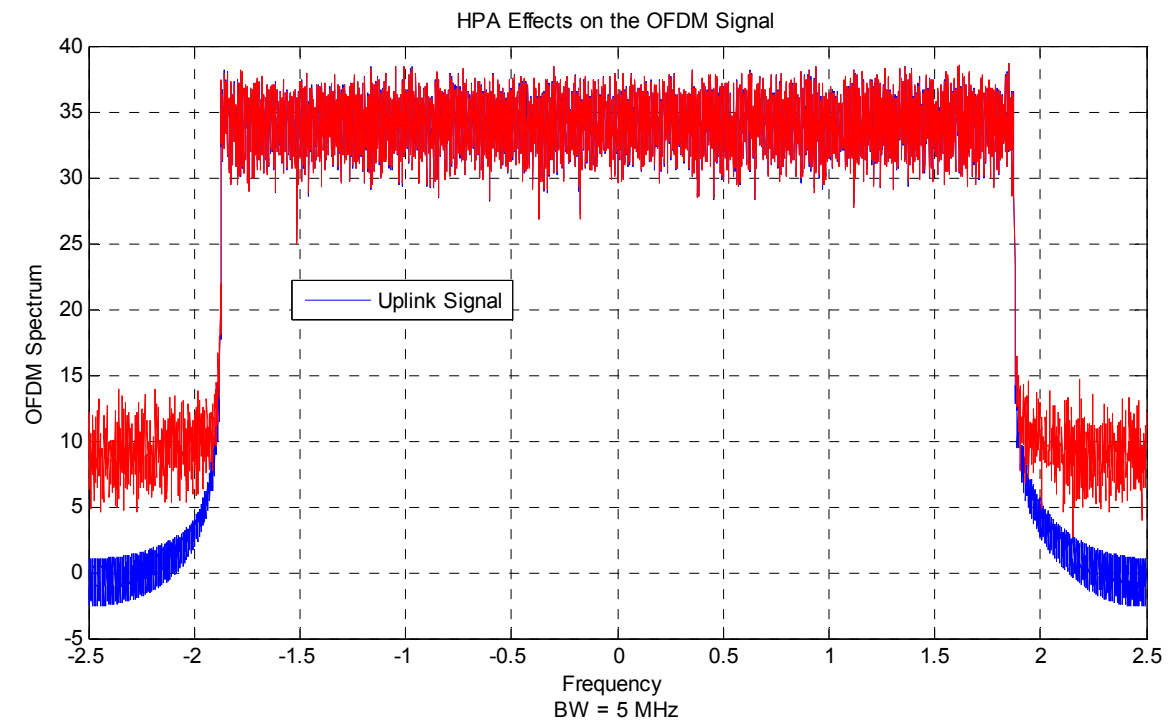

Fig. 4.28 16QAM OFDM Saturation Level $5 \mathrm{~dB}$ Relative to AM Average

An improvement in the downlink signal, notated in red, compared to the uplink signal in blue can be observed, compared to the downlink signal from Figure 4.26.

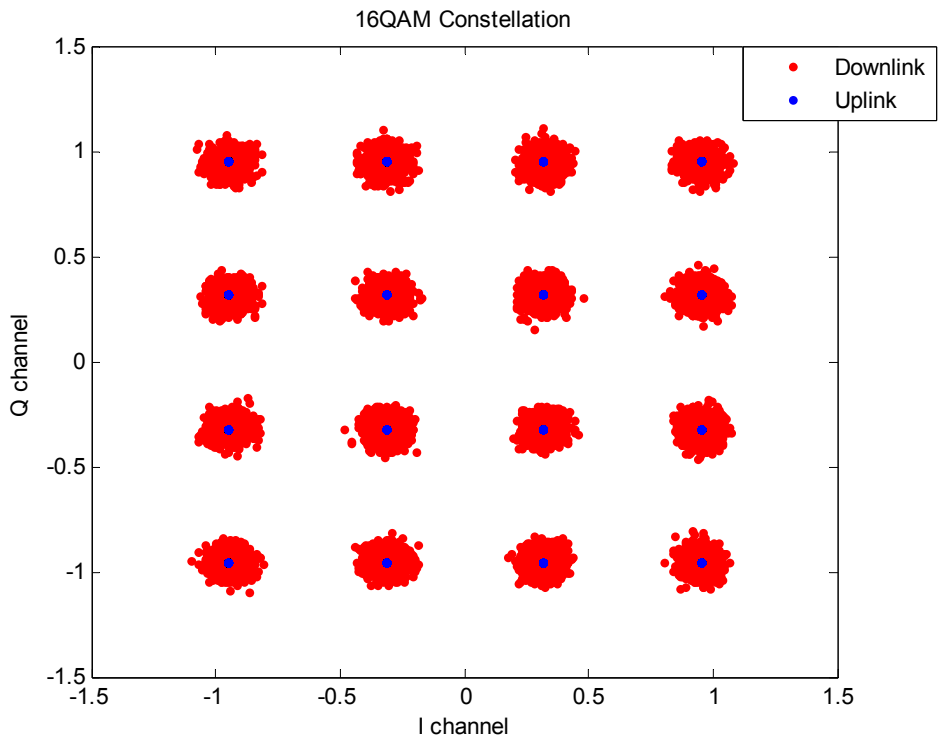

Fig. 4.29 16QAM OFDM Constellation Saturation Level 5 dB, K=2

The following tables show the bit error rate (BER) and modulation error rate (MER) measures at the receiver. 
Table 4.2 Modulation Error Rate, OFDM Satellite Channel $\frac{E_{b}}{N o}=25 d B$

\begin{tabular}{|c|c|c|}
\hline $\begin{array}{l}\text { Saturation } \\
\text { Level }(d B)\end{array}$ & $\begin{array}{c}\underline{O F D M} \underline{16 Q A M} \\
I F F T=2048, B W=5 \mathrm{MHz}\end{array}$ & $\begin{array}{c}\underline{O F D M Q P S K} \\
I F F T=1024, B W=5 \mathrm{MHz}\end{array}$ \\
\hline-1.5 & -11.9 & -11.8528 \\
\hline-1.0 & -12.509 & -12.5185 \\
\hline-0.5 & -13.1643 & -13.2822 \\
\hline 0 & -13.8105 & -13.8527 \\
\hline 0.5 & -14.6612 & -14.5654 \\
\hline 1.0 & -15.299 & -15.4131 \\
\hline 1.5 & -16.4789 & -16.2171 \\
\hline 2.0 & -17.38 & -17.2688 \\
\hline 2.5 & -18.4151 & -18.4182 \\
\hline 3.0 & -19.4805 & -19.8186 \\
\hline 3.5 & -20.7649 & -20.7854 \\
\hline 4.0 & -22.0552 & -22.1705 \\
\hline 4.5 & -23.6023 & -23.311 \\
\hline 5.0 & -24.9787 & -24.6751 \\
\hline
\end{tabular}

Table 4.3 Bit Error Rate, OFDM Satellite Channel $\frac{E_{b}}{N o}=25 d B$

\begin{tabular}{|l|c|l|}
\hline$\underline{\text { Saturation }}$ & $\underline{\text { OFDM 16QAM }}$ & $\underline{\text { OFDM QPSK }}$ \\
Level $(d B)$ & $I F F T=2048, B W=5 \mathrm{MHz}$ & $I F F T=1024, B W=5 \mathrm{MHz}$ \\
\hline
\end{tabular}




\begin{tabular}{|c|c|c|}
\hline-4.0 & 0.2642 & 0.003295 \\
\hline-3.5 & 0.2377 & 0.0022816 \\
\hline-3.0 & 0.2133 & 0.0011951 \\
\hline-2.5 & 0.19045 & 0.00043459 \\
\hline-2.0 & 0.15841 & 0.00076054 \\
\hline-1.5 & 0.12584 & 0.00010865 \\
\hline-1.0 & 0.097611 & 0.0001024 \\
\hline-0.5 & 0.069653 & $4.392 e^{-5}$ \\
\hline 0 & 0.044951 & $3.294 e^{-6}$ \\
\hline 0.5 & 0.026764 & $2.089 e^{-7}$ \\
\hline 1.0 & 0.014278 & $1.002 e^{-8}$ \\
\hline 1.5 & 0.0060261 & $1.0 e^{-8}$ \\
\hline 2.0 & 0.0015201 & $1.0 e^{-8}$ \\
\hline 2.5 & 0.00070575 & $1.0 e^{-8}$ \\
\hline 3.0 & $5.4289 e^{-5}$ & $1.0 e^{-8}$ \\
\hline 3.5 & $9.2454 e^{-6}$ & $1.0 e^{-8}$ \\
\hline 4.0 & $4.3759 e^{-7}$ & $1.0 e^{-8}$ \\
\hline 4.5 & $5.9221 e^{-8}$ & $1.0 e^{-8}$ \\
\hline 5.0 & $1.2562 e^{-8}$ & $1.0 e^{-8}$ \\
\hline
\end{tabular}

Figures 4.30 and 4.31 below show the graphs of the bit error rates for both QPSK and 16QAM OFDM over the satellite channel for different values of the Rician factor K. 


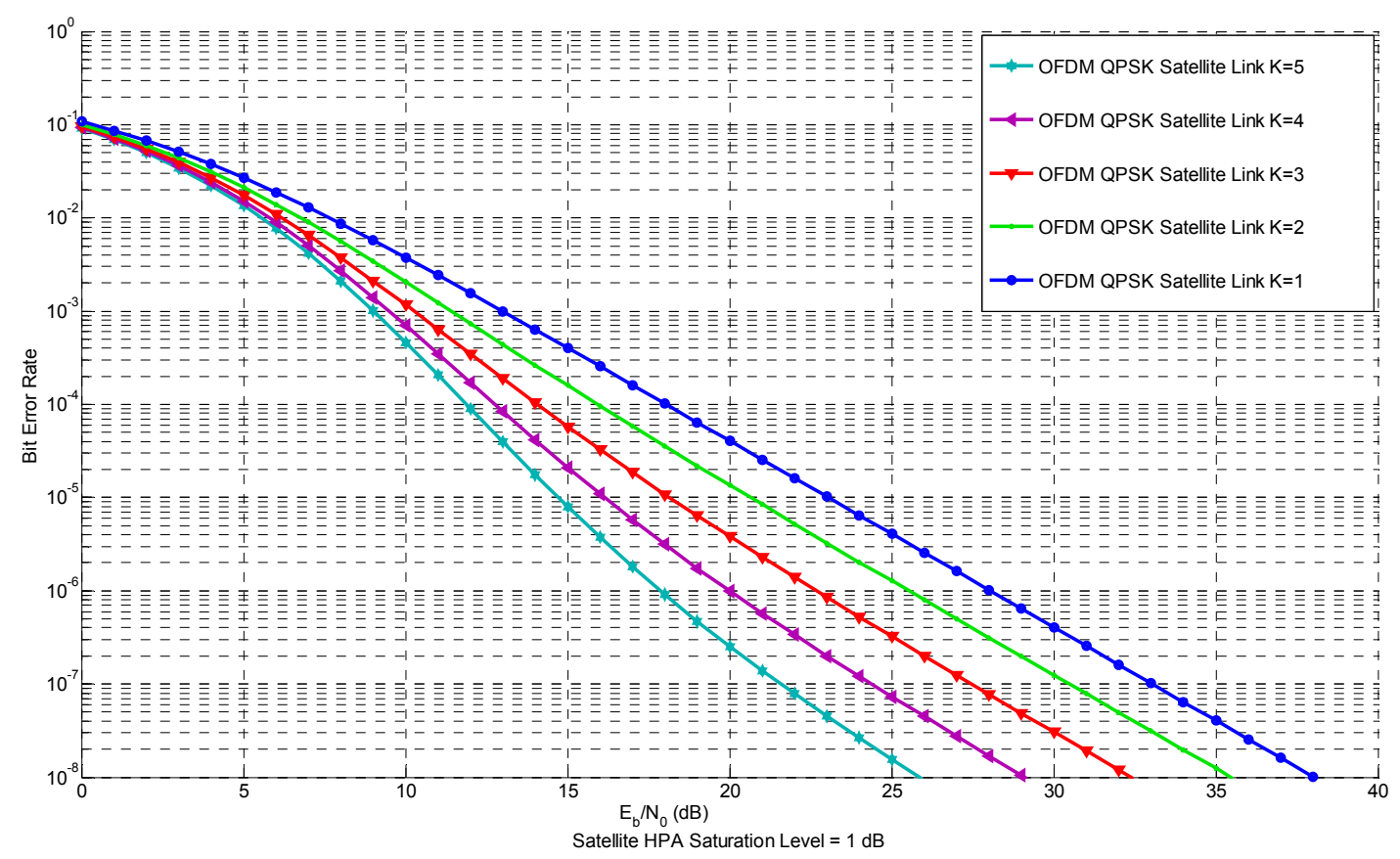

Fig. 4.30 BER Values QPSK OFDM Satellite Channel

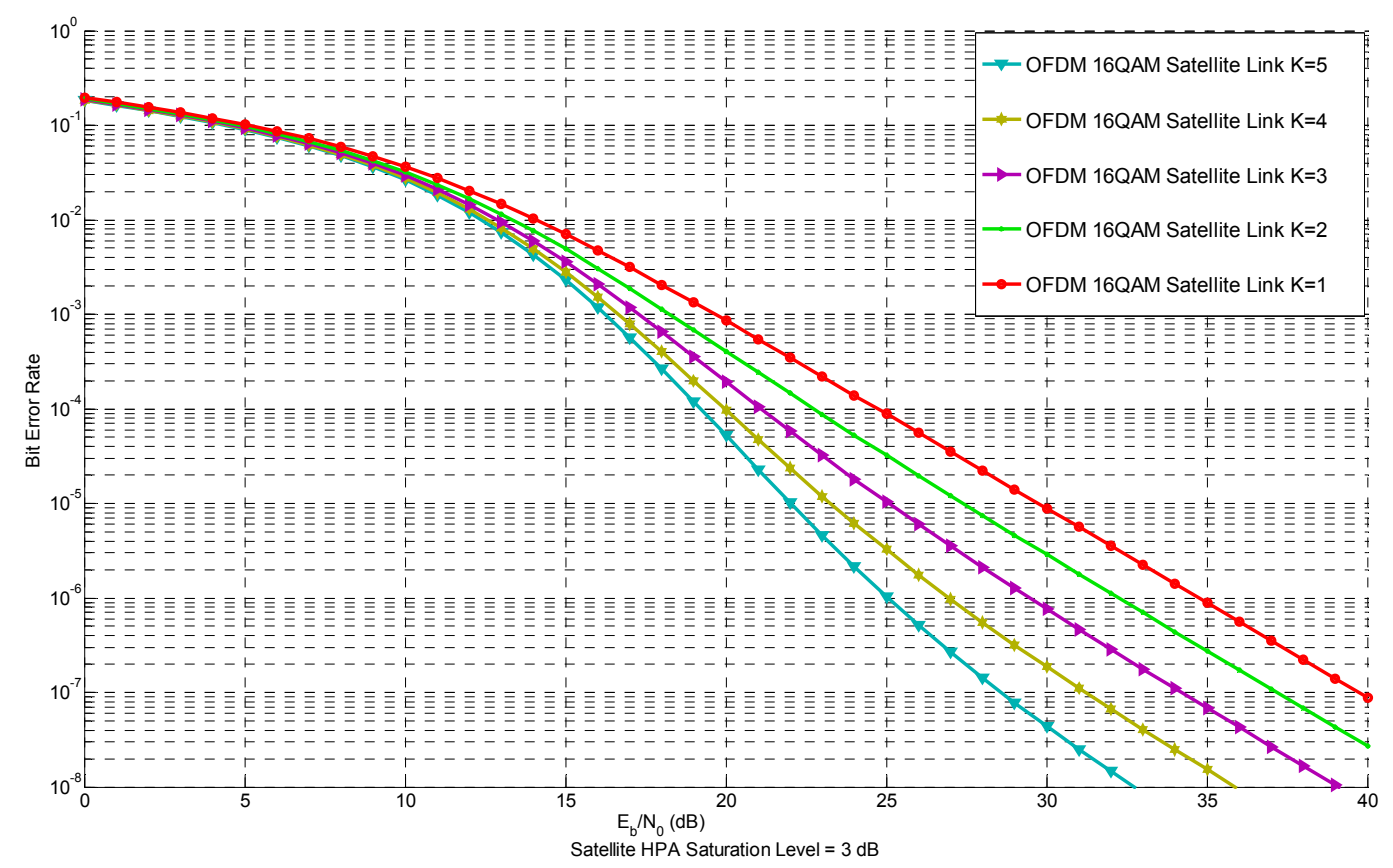

Fig. 4.31 BER Values 16QAM OFDM Satellite Channel 
If a channel coding block is added to the simulation, the BER results improve considerably, as shown in the following Figures 4.32 and 4.33 .

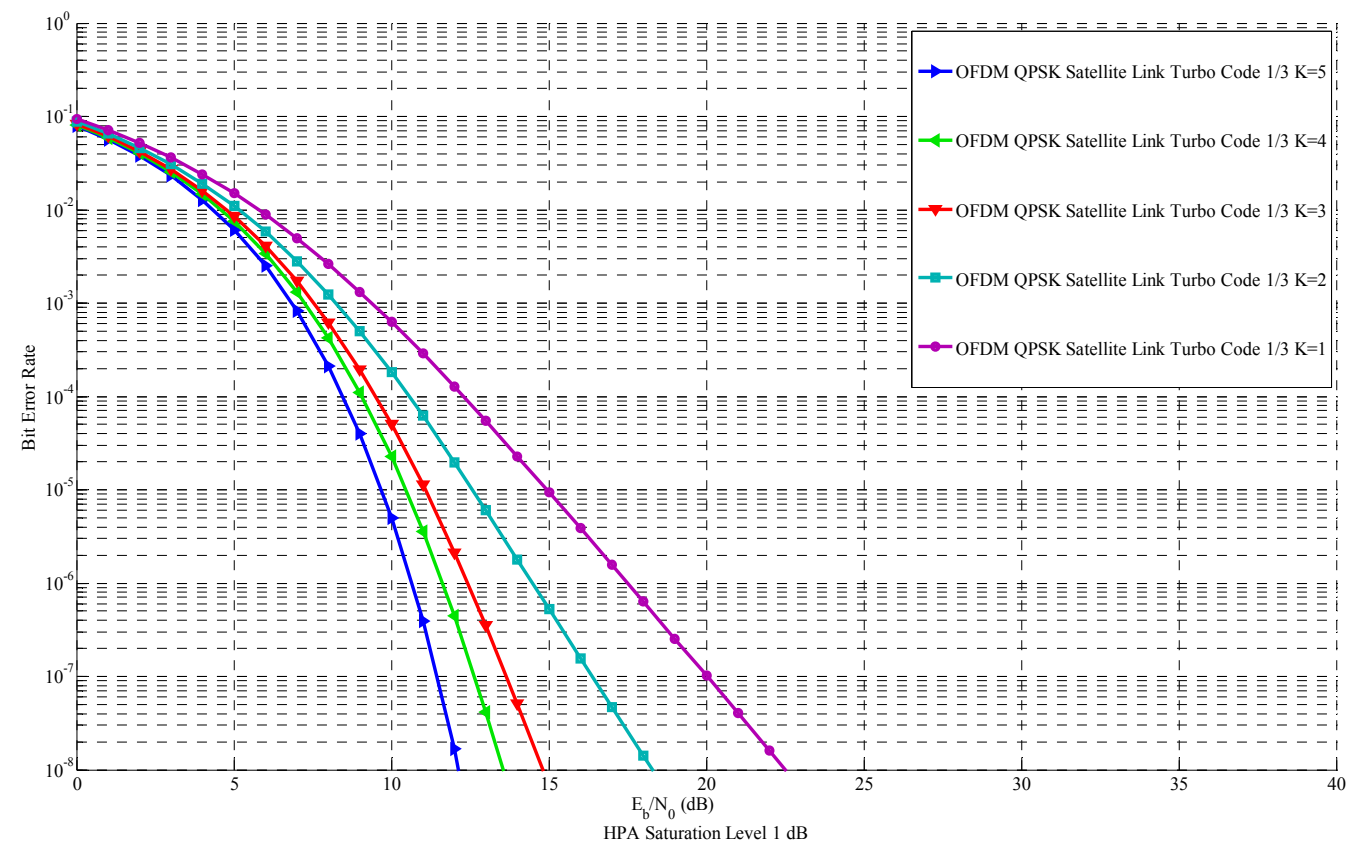

Fig. 4.32 BER Values QPSK OFDM, Using Turbo Code

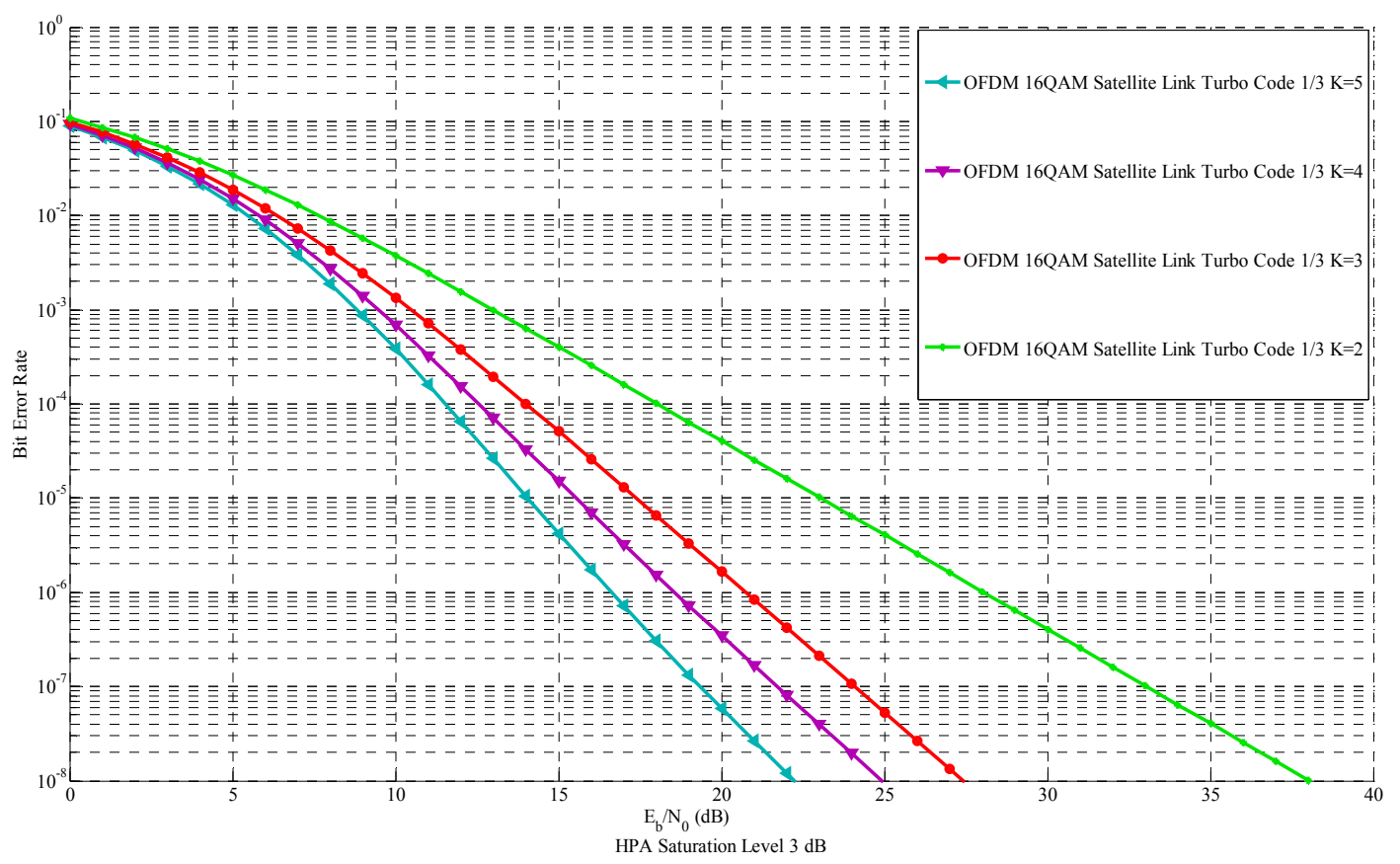

Fig. 4.33 BER Values 16QAM OFDM, Using Turbo Code 


\subsection{Summary}

This chapter put forth a time diversity mechanism to adapt the OFDM scheme for use over satellite links. In addition, the effects of non-linear distortions induced by satellite onboard high power amplifiers over OFDM transmission were analyzed. Finally, a system was simulated in which a Rician channel model is used in the satellite link. Analyses of both QPSK and 16QAM modulations were analyzed.

From the simulations results, it can be concluded that modeling the satellite channel as a Rician multipath fading channel is an accurate approach. This, as mentioned before, is valid only if there is a direct line of sight between the mobile user and the satellite. OFDM can be used as long as there is an appropriate transmission protection time and a good channel coding scheme, like turbo coding. 


\section{Chapter V. OFDM MIMO Space Diversity in Terrestrial Channels}

One of the most important recent contributions to the study of digital communications has been the adoption of multiple input multiple output (MIMO) techniques. MIMO improves the received signal quality and increases the data communication speed by employing digital signal processing techniques to shape and combine the transmitted signals from multiple wireless paths, created by the use of multiple transmit and receive antennas [2].

\subsection{MIMO Systems}

Spatial domain diversity can be achieved by using multiple antennas on the transmitter and/or receiver sides. The configuration of multiple antennas can be divided into three categories: (1) MISO (multiple input single output): uses more than one antenna at the transmitter and only one at the receiver; (2) SIMO (single input multiple output): uses one transmitting antenna and more than one receiving antenna; and (3) MIMO: uses more than one antenna at the transmitter and more than one at the receiver.

By using more than one transmit/receive antenna, multiple channels are employed between each pair of transmit and receive antennas (see Figure 5.1). The transmitted signal will travel through different channels to arrive at the receiver side. If one of the channels is sufficiently strong, the receiver will be able to recover the transmitted signal. If different channels are independent, then the probability of all channels failing is very small. 


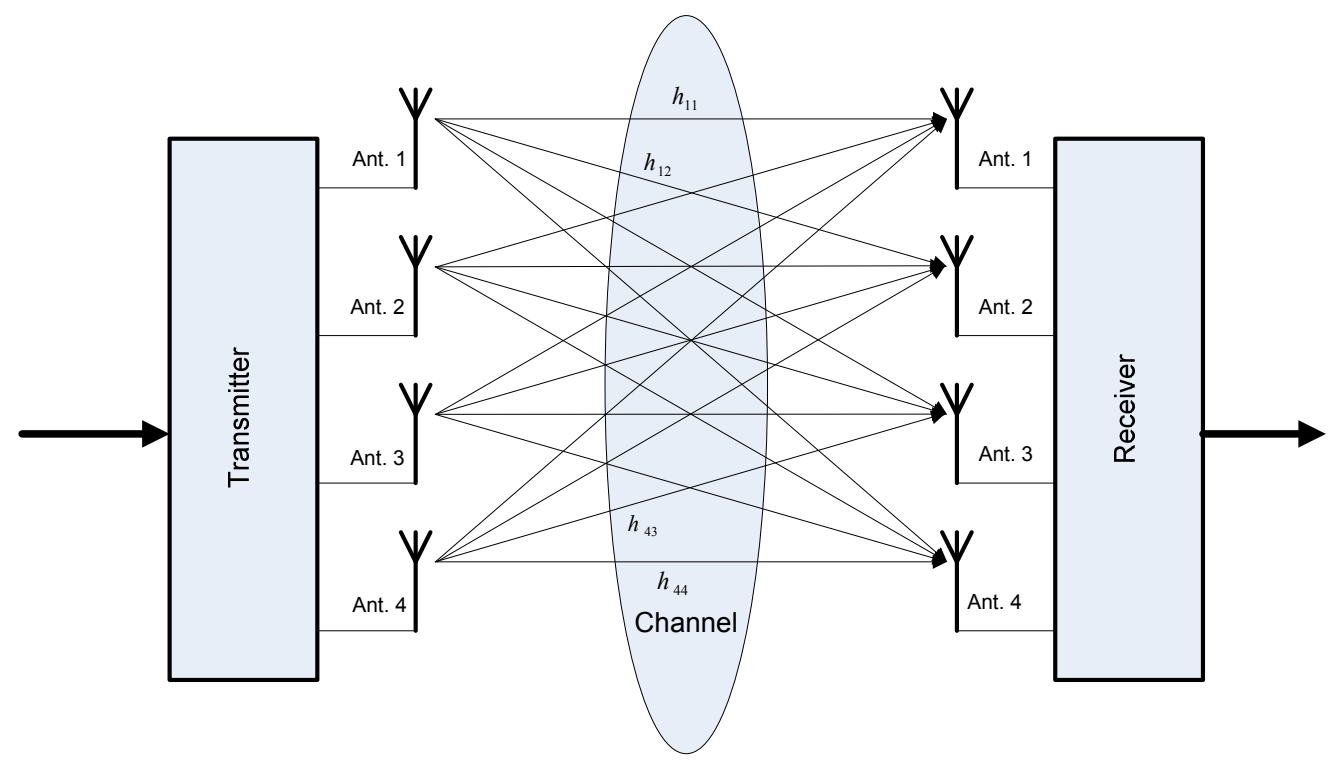

Fig. 5.1 MIMO Architecture

The greater the number of antennas, the greater the redundancy or diversity of the received signal will be. The low correlation of the different channels can be achieved by separating the antennas at both the transmitter and receiver. The correct antenna separation will depend on the scattering around the neighborhood of the antenna and the channel central frequency. In mobile environments, the separation can range from onehalf to one carrier wavelength.

In MIMO systems, both transmit and receive diversities are realized. The overall signal redundancy, or diversity order, is the product of the number of transmit and receive antennas [64]. The MIMO configuration can be realized through design. The designs differ in the antenna configuration at the receiver and transmitter, in addition to the particular form of performance improvement it is designed to obtain. The capacity of a MIMO system increases at least linearly with the minimum number of transmit or receive antennas. 
In a MIMO system with $M_{T}$ transmit antennas and $M_{R}$ receive antennas, the channel coefficient between transmit antenna $i$ and receive antenna $j$ will be $h_{i, j}$

These $h$ coefficients are modeled as circularly symmetric complex Gaussian random variables, with zero mean and variance equal to one. The MIMO trans-receiver can be modeled by the following equation [2]:

$$
Y=\sqrt{\frac{\rho}{M_{T}}} X H+Z
$$

where $X=\left\lfloor x_{1}, x_{2}, \ldots . x_{M_{T}}\right\rfloor$ is a signal vector transmitted by the $M_{T}$ transmit antennas;

$$
\begin{aligned}
& Y=\left[y_{1}, y_{2}, \ldots . y_{M_{R}}\right] \text { is a signal vector received by the } M_{R} \text { receive antennas; and } \\
& Z=\left[z_{1}, z_{2}, \ldots z_{M_{R}}\right] \text { is a noise vector whose elements are modeled as }
\end{aligned}
$$

independent circularly symmetric complex Gaussian random variables, with zero mean and variance equal to one.

The channel coefficient matrix $H=\left\{h_{i, j}: 1 \leq i \leq M_{T}, 1 \leq j \leq M_{R}\right\}$ is assumed to be known at the receiver side. The signal vector is assumed to satisfy the energy constraint $E\|X\|_{F}{ }^{2}=M_{T}$, where $\|X\|_{F}$ is the Frobenius norm of $X$ and defined by:

$\|X\|_{F}^{2}=\sum_{i=1}^{M_{T}}\left|x_{i}\right|^{2}$

In (5.1), the factor $\sqrt{\frac{\rho}{M_{T}}}$ ensures that $\rho$ is the average $S N R$ at each receiver antenna, and it is independent of the number of transmitting antennas. If the input signal $X$ is a circularly symmetric complex Gaussian random vector with zero mean and 
variance $E\left\{X^{H} X\right\}=Q$, then the output signal is also a circularly symmetric complex Gaussian random vector, with zero mean and variance equaling, as follows:

$E\left\{Y^{H} Y\right\}=\frac{\rho}{M_{T}} H^{H} Q \cdot H+I_{M_{R}}$

$I_{M_{T}}$ is an identity matrix of size $M_{R}$ by $M_{R}$. Hence, for any given channel $H$, the mutual information between the input $X$ and output $Y$ is:

$I(X ; Y \mid H)=\log _{2} \operatorname{det}\left(I_{M_{T}}+\frac{\rho}{M_{T}} H^{H} Q \cdot H\right)$

If the transmitter has no prior knowledge of the channel conditions, each transmitting antenna can be treated equally and allocated the same weight. The variance of the input signal vector over $M_{T}$ transmit antennas should be an identity matrix. The maximal mutual information will be:

$\log _{2} \operatorname{det}\left(I_{M_{R}}+\frac{\rho}{M_{T}} \widetilde{H}^{H} \widetilde{H}\right)$

If the channel is memory-less, then $H$ changes independently from each use of the channel to another. The average capacity of the MIMO system will be:

$$
C=E_{H}\left\{\log _{2} \operatorname{det}\left(I_{M_{R}}+\frac{\rho}{M_{T}} H^{H} H\right)\right\}
$$

The expectation is taken over the fading channel $H$. From (5.6), it can be observed that when $M_{T}=M_{R}=1$, the capacity is reduced to a conventional single input single output (SISO) system.

In a MIMO system $M_{T}=M_{R}>1$, for large number of transmitting antennas $M_{T}$, the capacity in (5.6) is: 
$C \rightarrow \log _{2} \operatorname{det}\left(I_{M_{R}}+\rho \cdot I_{M_{R}}\right)=M_{R} \log _{2}(1+\rho)$

$\mathrm{C}$ increases linearly with the number of receiving antennas. The analysis is also true for any systems in which $M_{T} \geq M_{R}$. The capacity of a multiple antenna system increases at least linearly with the number of transmit and receive antennas.

\subsection{MIMO-OFDM System}

A MIMO-OFDM offers both spatial and frequency diversity in broadband communications. In general, the system is composed of $M_{T}$ transmitting antennas, $M_{R}$ receiving antennas. and $N$ OFDM sub-carriers (as indicated in Figure 5.2). The frequency selective fading channel [69] between each pair of transmit and receive antennas has $L$ independent delay paths and the same power delay profile. The MIMO channel is constant over each OFDM block period [67, 68].

The channel impulse response from transmit antenna $i$ and receive antenna $j$ can be modeled as:

$h_{i, j}=\sum_{l=0}^{L-1} \alpha_{i, j}(l) \cdot \delta\left(\tau-\tau_{l}\right)$,

where $\tau_{l}$ is the delay of the lth path, and $\alpha_{i, j}(l)$ is the complex amplitude of the lth path between transmit antenna $i$ and receive antenna $j$. The time delay $\tau_{l}$ and variance $\delta^{2}{ }_{l}$ are assumed to be the same for each transmit-receive channel link. The power of the $L$ paths are normalized, such that $\sum_{l=0}^{L-1} \delta^{2}{ }_{l}=1$. From (5.8), the channel frequency response is given by: 
$H_{i, j}(f)=\sum_{l=0}^{L-1} \alpha_{i, j}(l) \cdot e^{-j 2 \pi \cdot f \cdot \tau_{l}}, j=\sqrt{-1}$

The input bit sequence from a channel encoder is divided into $b$ bit long segments, thus forming $2^{b}$ source symbols. These symbols are parsed into blocks and mapped onto a space-frequency (SF) code word that is transmitted over the $M_{T}$ transmit antennas. Each of the SF code words can be defined as a $N \times M_{T}$ matrix as follows:

$$
C=\left[\begin{array}{cccc}
c_{1}(0) & c_{2}(0) & \ldots . & c_{M_{T}}(0) \\
c_{1}(1) & c_{2}(1) & \ldots . & c_{M_{T}}(1) \\
\cdot & \cdot & . & \cdot \\
c_{1}(\dot{N}-1) & c_{2}(\dot{N}-1) & \ldots & c_{M_{T}}(\dot{N}-1)
\end{array}\right]
$$

$c_{i}(n)$ denotes the channel symbol transmitted over the $n t h$ sub-carrier by antenna $i$, and $N$ is the number of sub-carriers in the OFDM scheme. The OFDM transmitter applies an $N$ point IFFT to each column of the matrix $C$. After adding a cyclic prefix, the OFDM symbol corresponding to the $i t h\left(i=1,2, \ldots . . M_{T}\right)$ column of $C$ is transmitted by transmit antenna $i$. All of the $M_{T}$ OFDM symbols are transmitted at the same time from different transmit antennas.

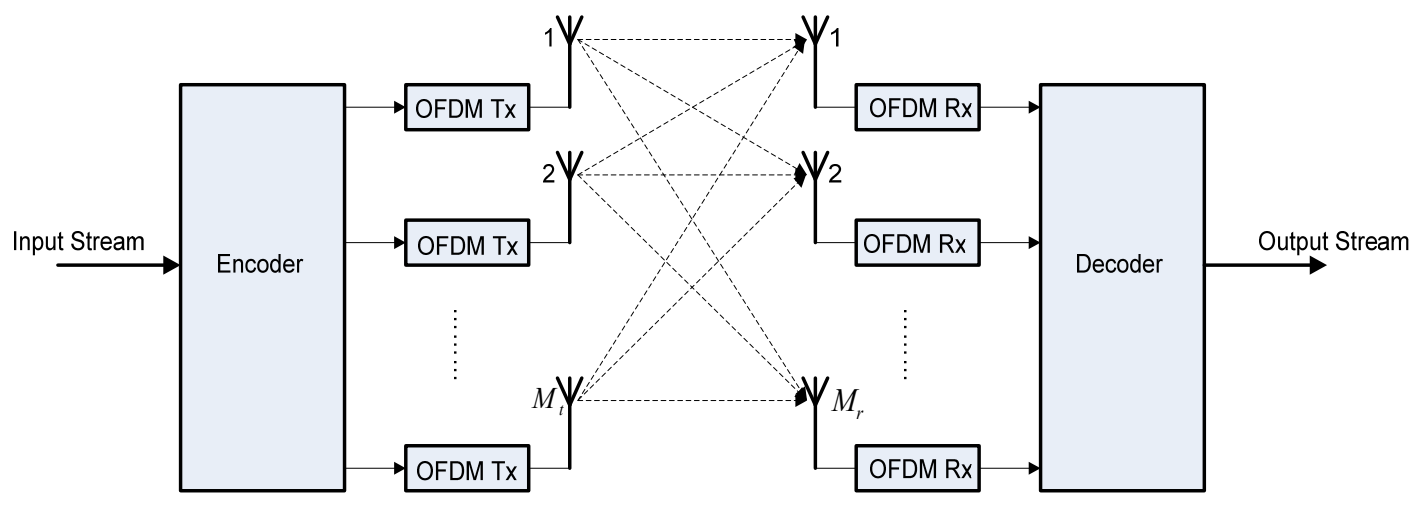

Fig. 5.2 MIMO-OFDM System 
At the receiver, after filtering, removing the cyclic prefix, and applying FFT, the received signal at the $n t h$ sub-carrier at receive antenna $j$ is:

$$
\begin{aligned}
& y_{j}(n)=\sqrt{\frac{\rho}{M_{T}}} \sum_{i=1}^{M_{T}} c_{i}(n) \cdot H_{i, j}(n)+z_{j}(n), \\
& \text { where } H_{i, j}(n)=\sum_{l=0}^{L-1} \alpha_{i, j}(l) \cdot e^{-j 2 \pi \cdot n \Delta f \cdot \tau_{l}}
\end{aligned}
$$

is the channel frequency response at the nth sub-carrier between transmit antenna

$i$ and receive antenna $j, \Delta f=\frac{1}{T}$ is the sub-carrier separation in frequency domain, and $T$ is the OFDM symbol period. The channel state information $H_{i, j}(n)$ is known at the receiver, but not at the transmitter. The term $z_{j}(n)$ in (5.11) denotes the additive complex Gaussian noise with zero mean and variance of one at the nth sub-carrier at receive antenna $j$. The noise samples $z_{j}(n)$ are uncorrelated for different values of $j$ and $n$.

The factor $\sqrt{\frac{\rho}{M_{T}}}$ guarantees that $\rho$ is the average SNR at each receive antenna, independent of the number of transmit antennas [66].

\subsection{MIMO Alamouti Encoder}

Recent research in MIMO systems shows that space time coding (STC) is effective to reduce fading effects in wireless channels because it provides diversity and improves BER performance [70]. Alamouti presented an excellent spatial scheme for transmissions with two transmit antennas that improve the quality of the received signal (see Figure 5.3). It uses simple processing at the transmitter and linear decoding at the receiver [62]. This scheme improves error performance, data rate and capacity [65]. The Alamouti 
scheme includes two transmit and four receive antennas, although it can be designed to accommodate any number of transmit and receive antennas. In a one transmitter, one receiver scheme, the symbols $s_{0}, s_{1}, \ldots$ are transmitted at symbol periods $t, t+t, t+2 T, \ldots$. , respectively.

In a two transmitter Alamouti encoder scheme, the symbols $s_{0}$ and $s_{1}$ are transmitted simultaneously from two transmit antennas $T_{X_{1}}$ and $T_{X_{2}}$ respectively, at symbol period $t$. At the next symbol period, $t+T, T_{X_{1}}$ transmits symbol $-s_{1}{ }^{*}$, and $T_{X_{2}}$ transmits $-s_{0}{ }^{*}$. This STC coding is known as G2 STC [63].
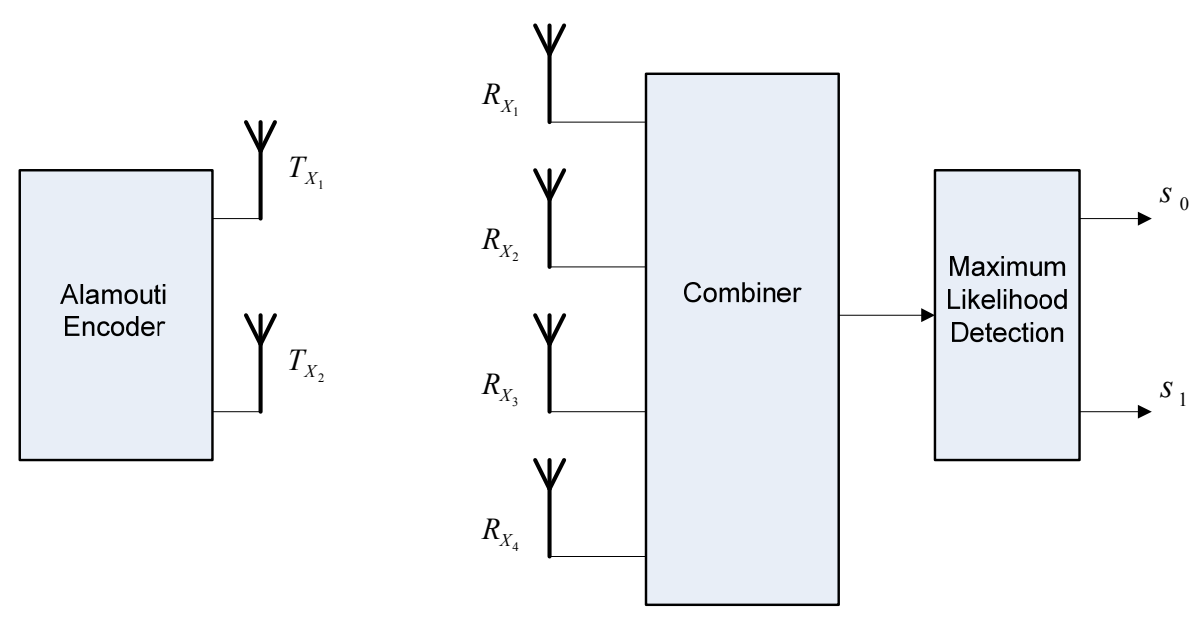

Fig. 5.3 Alamouti Scheme $2 \times 4$

If the number of receive antennas is increased, the BER improves, while the complexity simultaneously increases [61]. From Figure 5.4, it can be observed that the $2 \times 4$ scheme performs better than the others.

The BER of $10^{-4}$ is achieved at $\mathrm{SNR}=2$ for a $2 \times 4$ system. On the other hand, the same $\mathrm{BER}$ is achieved at $\mathrm{SNR}=8$ for a $2 \times 2$ system, and at $\mathrm{SNR}=16$ in a $2 \times 1$ system. An 
Alamouti of $2 \times 4$ outputs two streams of symbols, and the outputs are fed to identical transmit chains.

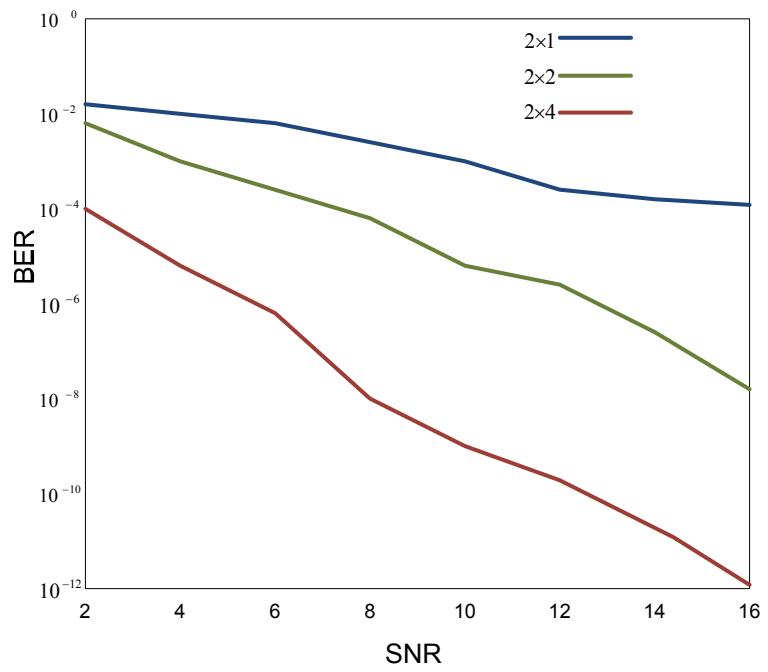

Fig. 5.4 Alamouti Schemes BER Comparison

\subsection{Simulation Results}

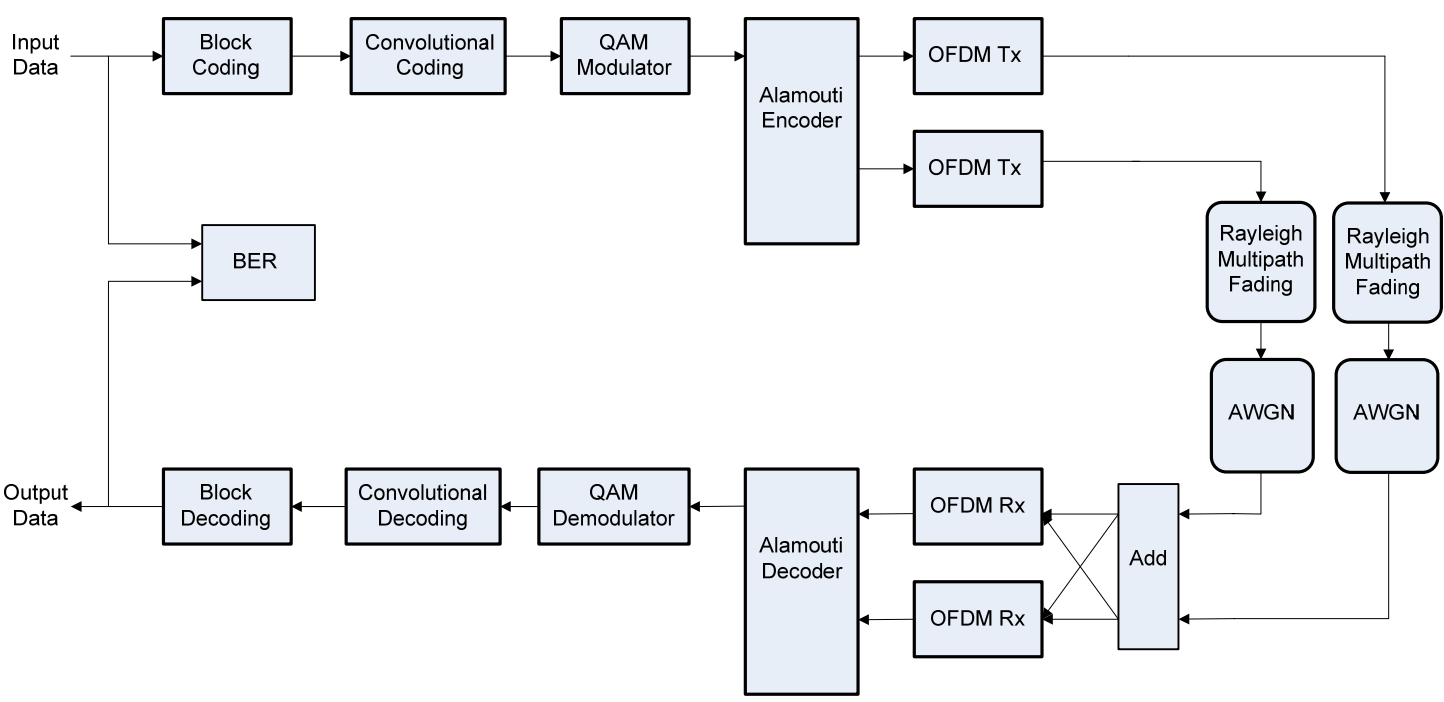

Fig. 5.5 MIMO OFDM Simulation Block Diagram

Figure 5.5 shows the simulation block diagram in MatLab used to model the terrestrial channel MIMO OFDM link. The input random bits are fed into a block coding 
block, which adds redundant bits in order to perform channel block coding. This block converts the input bits into blocks of fixed length and adds redundant bits to each block, so its output block will be longer than the input block.

The output of the block coding is fed into a convolutional coding block that performs another type of channel coding, known as the Trellis algorithm. This type is different from block coding, in that the input bits are not divided into blocks of fixed length; instead, the input bits are encoded continuously. The combination of block codes and convolutional codes allows a robust combination when detecting and correcting errors at the receiver, which uses a Viterbi decoder to decode the Trellis algorithm and a block decoding to decode the block codes. This process is known as concatenated coding.

The output of concatenated coding is modulated using a QAM modulator, and then fed into an Alamouti encoder to perform the MIMO technique, in this case, a $2 \times 2$ system. The $2 \times 2$ system uses two transmit and two receive antennas that perform the space diversity. The Alamouti encoder outputs are modulated using two OFDM transmitters and two antennas, which transmit the OFDM signal through a Rayleigh multipath channel.

The simulation parameters are the following:

Bandwidth: $5 \mathrm{MHz}$

Central frequency: $1.5 \mathrm{GHz}$

OFDM sub-carrier spacing: $\Delta f=15 \mathrm{KHz}$

OFDM IFFT size: 2048 for 16QAM

OFDM IFFT size: 1024 for QPSK

Number of OFDM symbols: 12 
Cyclic prefix duration: $16.67 \mu \mathrm{s}$

Maximum Doppler shift: $15 \mathrm{~Hz}$

Error correction rates:

- BPSK: $1 / 2$

- QPSK: $1 / 2,3 / 4$

-16QAM: $1 / 2,3 / 4$

- 64QAM: $2 / 3$

Number of transmit antennas: 2

Number of receive antennas: 2

Channel type: Multipath Rayleigh Fading

Block coding: 188/204

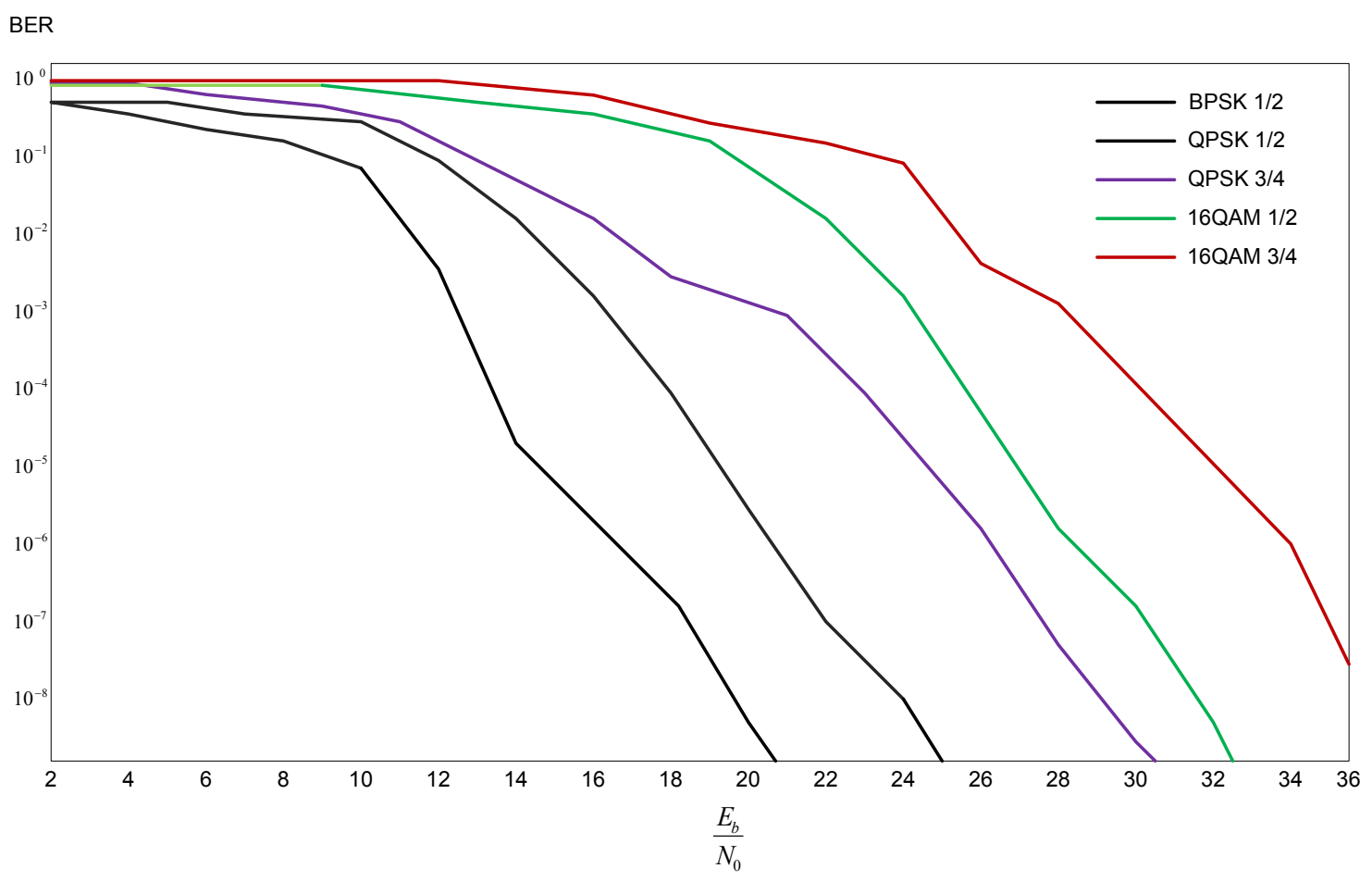

Fig. 5.6 $\frac{E_{b}}{N_{0}}$ versus BER Results for BPSK, QPSK and 16QAM 
Above, Figure 5.6 shows the functions of $\frac{E_{b}}{N_{0}}$ versus BER for different modulations and error correction codes. BPSK $1 / 2$ shows the best performance, compared to the others modulation schemes, but it lacks the capacity to transmit high data rates. QPSK $1 / 2$ and QPSK $3 / 4$ can accommodate larger data rates, and their BER performances are good in Rayleigh fading channels with OFDM transmissions.

16QAM $1 / 2$ can perform with acceptable BER values over Rayleigh channels, and it is capable of carrying high data rates, depending on the error correction code that is selected. The figure shows that BER is better with 16QAM 1/2, compared to 16QAM 3/4. This is because with $1 / 2$, the number of redundant bits is considerably higher than in the 16 QAM $3 / 4$ case. However, even with the high amount of redundant bits, 16QAM $1 / 2$ is capable of accommodating high data rates needed in $4 \mathrm{G}$ systems.

The following tables show the results for all the cases presented in Figure 5.6.

Table 5.1 BER BPSK $1 \frac{1}{2} B W=5 M H z$

\begin{tabular}{|c|c|}
\hline$\frac{E_{b}}{N_{0}}[d B]$ & $\begin{array}{l}\text { Bit Error Rate } \\
\text { BER }\end{array}$ \\
\hline 4 & .465 \\
\hline 6 & .402 \\
\hline 8 & .209 \\
\hline 10 & .0182 \\
\hline 12 & .00039 \\
\hline 14 & $2.14 e^{-5}$ \\
\hline
\end{tabular}




\begin{tabular}{|c|c|}
\hline 16 & $3.25 e^{-6}$ \\
\hline 18 & $4.37 e^{-7}$ \\
\hline 20 & $1.75 e^{-8}$ \\
\hline
\end{tabular}

Table 5.2 BER QPSK $1 / 2 B W=5 \mathrm{MHz}$

\begin{tabular}{|c|c|}
\hline$\frac{E_{b}}{N_{0}}[d B]$ & Bit Error Rate \\
\hline 8 & .458 \\
\hline 10 & .445 \\
\hline 12 & .295 \\
\hline 14 & .064 \\
\hline 16 & .0025 \\
\hline 18 & $1.74 e^{-5}$ \\
\hline 20 & $1.23 e^{-6}$ \\
\hline 22 & $3.49 e^{-7}$ \\
\hline 24 & $5.26 e^{-8}$ \\
\hline & \\
\hline
\end{tabular}

Table 5.3 BER QPSK $3 / 4 B W=5 M H z$

\begin{tabular}{|c|c|}
\hline$\frac{E_{b}}{N_{0}}[d B]$ & Bit Error Rate \\
\hline 6 & BER \\
\hline
\end{tabular}




\begin{tabular}{|c|c|}
\hline 8 & .484 \\
\hline 10 & .476 \\
\hline 12 & .462 \\
\hline 14 & .443 \\
\hline 16 & .354 \\
\hline 18 & .165 \\
\hline 20 & .0242 \\
\hline 22 & .0009 \\
\hline 24 & $7.48 e^{-5}$ \\
\hline 26 & $5.95 e^{-6}$ \\
\hline 28 & $6.83 e^{-7}$ \\
\hline 30 & $3.48 e^{-8}$ \\
\hline
\end{tabular}

Table 5.4 BER 16QAM $1 / 2 B W=5 \mathrm{MHz}$

\begin{tabular}{|c|c|}
\hline$\frac{E_{b}}{N_{0}}[d B]$ & Bit Error Rate \\
\hline 6 & .495 \\
\hline 8 & .483 \\
\hline 10 & .482 \\
\hline 12 & .481 \\
\hline 14 & .476 \\
\hline 16 & .457 \\
\hline
\end{tabular}




\begin{tabular}{|c|c|}
\hline 18 & .325 \\
\hline 20 & .127 \\
\hline 22 & .01243 \\
\hline 24 & .00035 \\
\hline 26 & $4.92 e^{-5}$ \\
\hline 28 & $3.94 e^{-6}$ \\
\hline 30 & $4.36 e^{-7}$ \\
\hline 32 & $5.64 e^{-8}$ \\
\hline
\end{tabular}

Table 5.5 BER 16QAM $3 / 4 B W=5 \mathrm{MHz}$

\begin{tabular}{|c|c|}
\hline$\frac{E_{b}}{N_{0}}[d B]$ & $\begin{array}{l}\text { Bit Error Rate } \\
\text { BER }\end{array}$ \\
\hline 6 & .492 \\
\hline 8 & .495 \\
\hline 10 & .491 \\
\hline 12 & .482 \\
\hline 14 & .481 \\
\hline 16 & .480 \\
\hline 18 & .475 \\
\hline 20 & .444 \\
\hline 22 & .394 \\
\hline 24 & .337 \\
\hline
\end{tabular}




\begin{tabular}{|c|c|}
\hline 26 & .0095 \\
\hline 28 & .0047 \\
\hline 30 & .00031 \\
\hline 32 & $4.83 e^{-5}$ \\
\hline 34 & $2.32 e^{-6}$ \\
\hline 36 & $8.78 e^{-7}$ \\
\hline 38 & $5.44 e^{-8}$ \\
\hline
\end{tabular}

The signal spectrum for the OFDM 16QAM transmitted by Antennas 1 and 2 are shown in Figure 5.7. The received spectrum is shown in Figure 5.8; note the signal attenuation due to the Rayleigh channel.

The 16QAM and QPSK transmitted and received constellations are shown in Figures 5.9 and 5.10, respectively.

Note that the noise affects the phases more prominently in 16QAM due to the phase's proximity. In addition, the signal eye diagram for QPSK and 16QAM modulations can be found in Figures 5.11 and Fig. 5.12. 


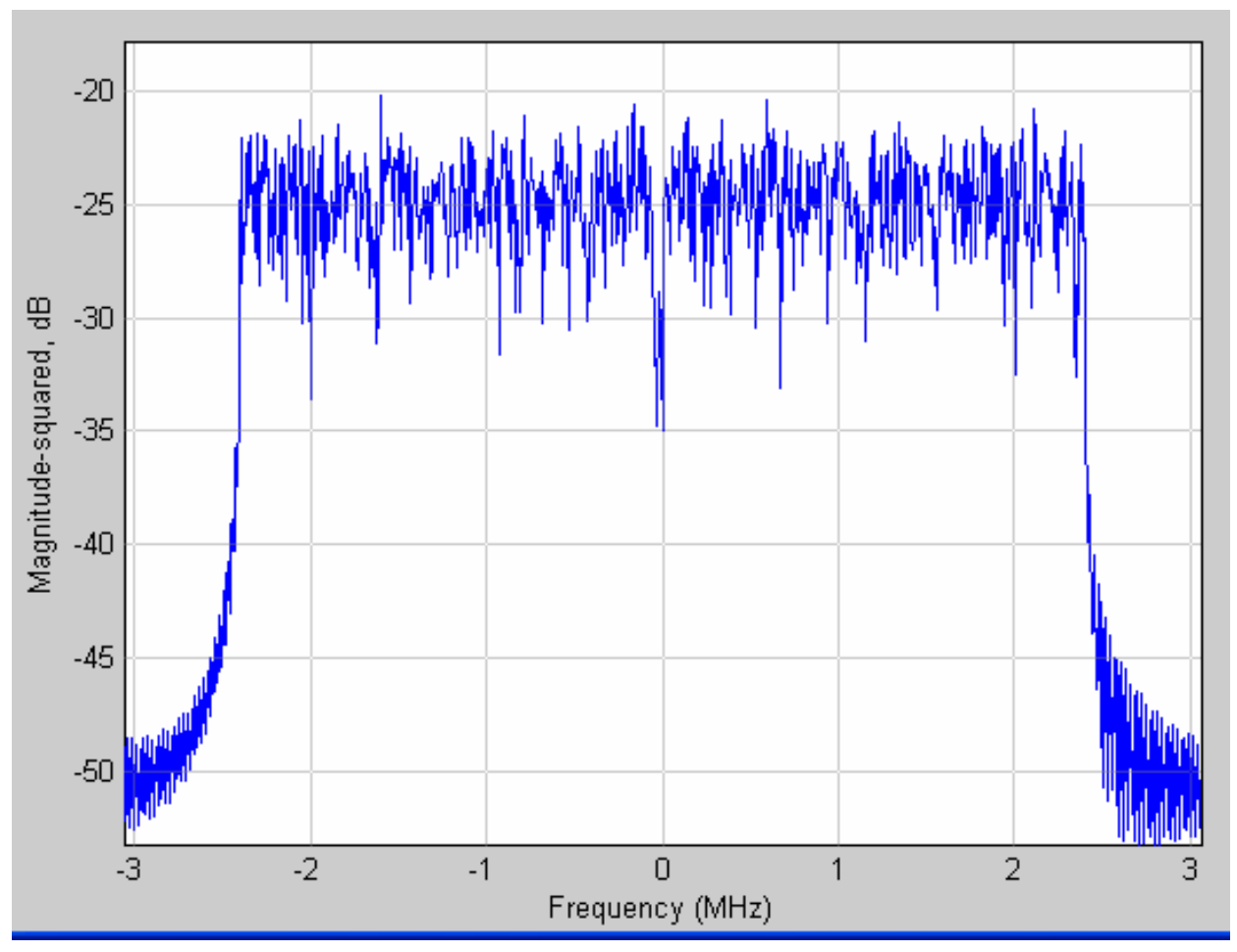

Fig. 5.7 OFDM 16QAM 3/4 Transmitted Spectrum Antenna 1

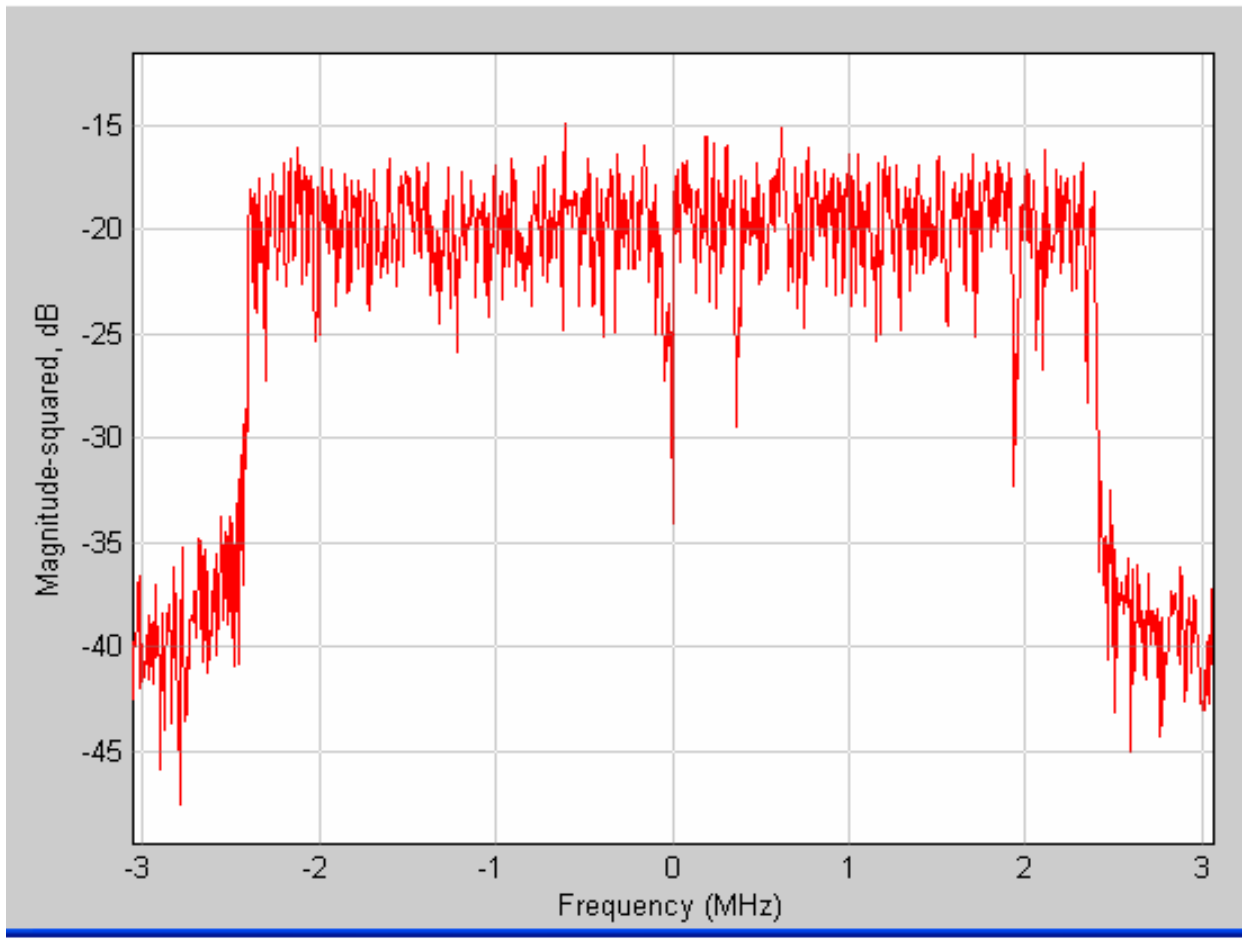

Fig. 5.8 OFDM 16QAM 3/4 Received Spectrum BER $=4.83 e^{-5}$ 

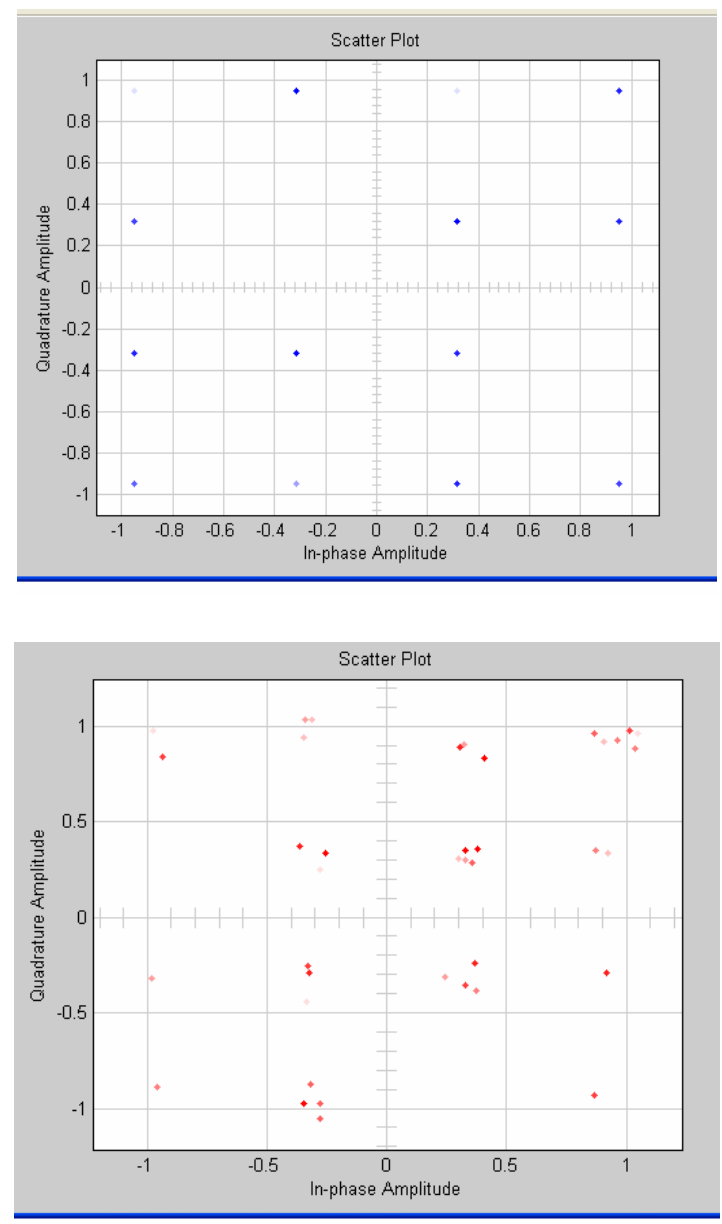

Fig. 5.9 16QAM 3/4 Transmitted (Blue) and Received (Red) Constellations BER = $4.83 e^{-5}$
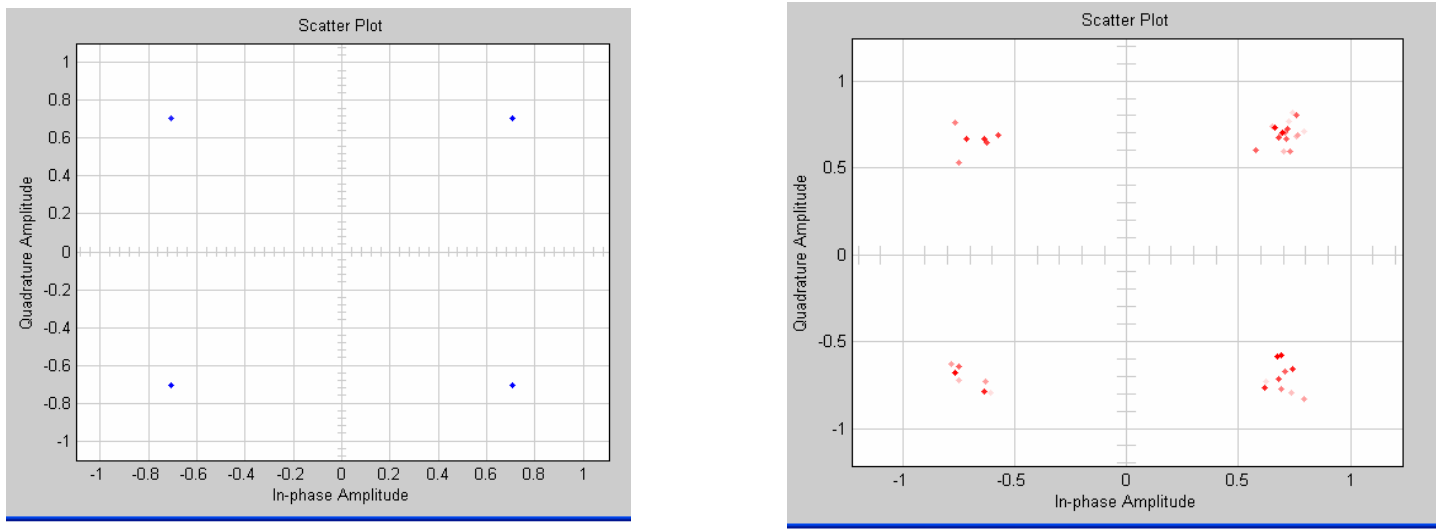

Fig. 5.10 QPSK 3/4 Transmitted and Received Constellations BER $=7.48 e^{-5}$ 

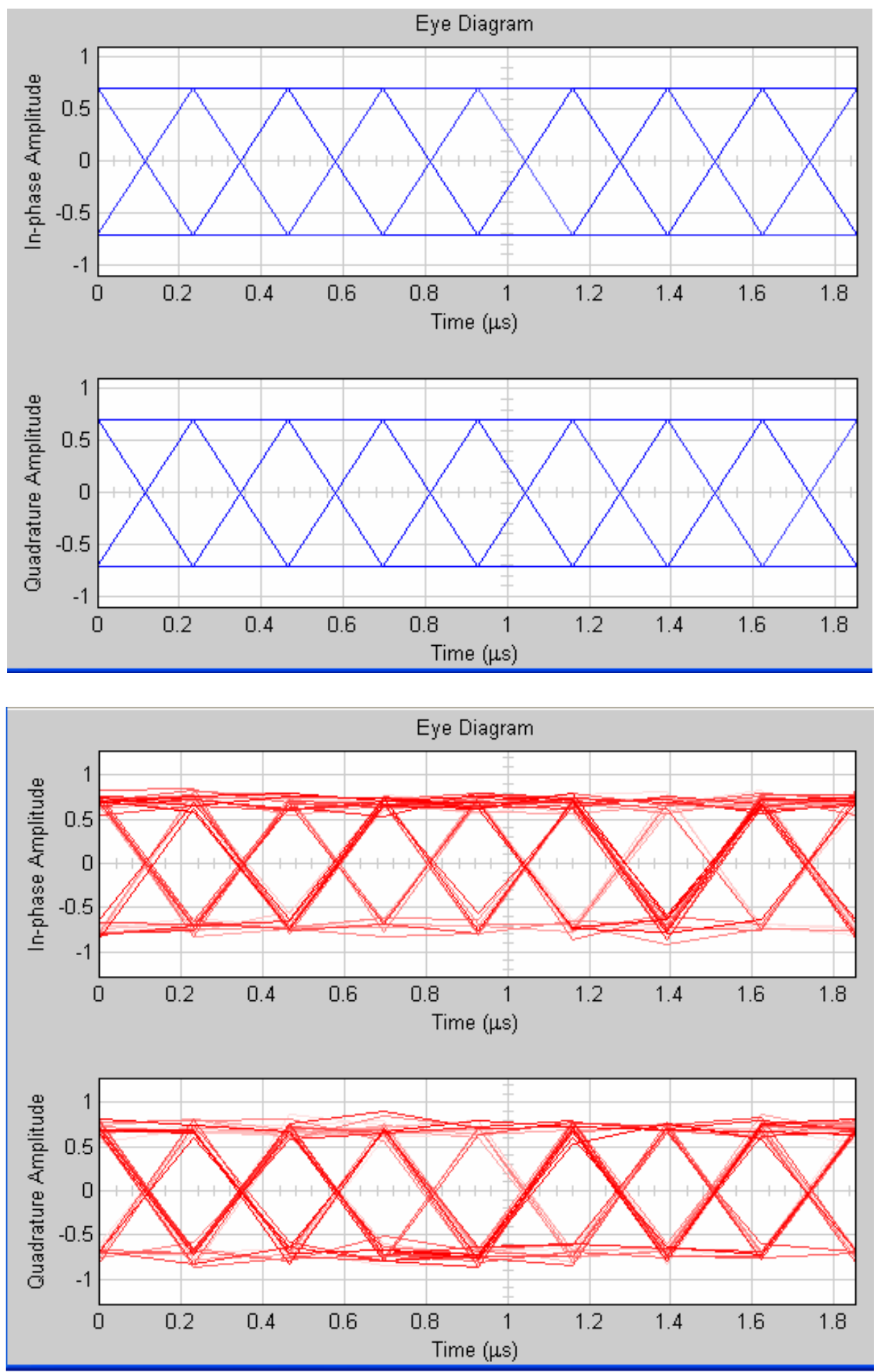

Fig. 5.11 QPSK 3/4 Transmitted and Received Eye Diagrams BER $=7.48 e^{-5}$ 

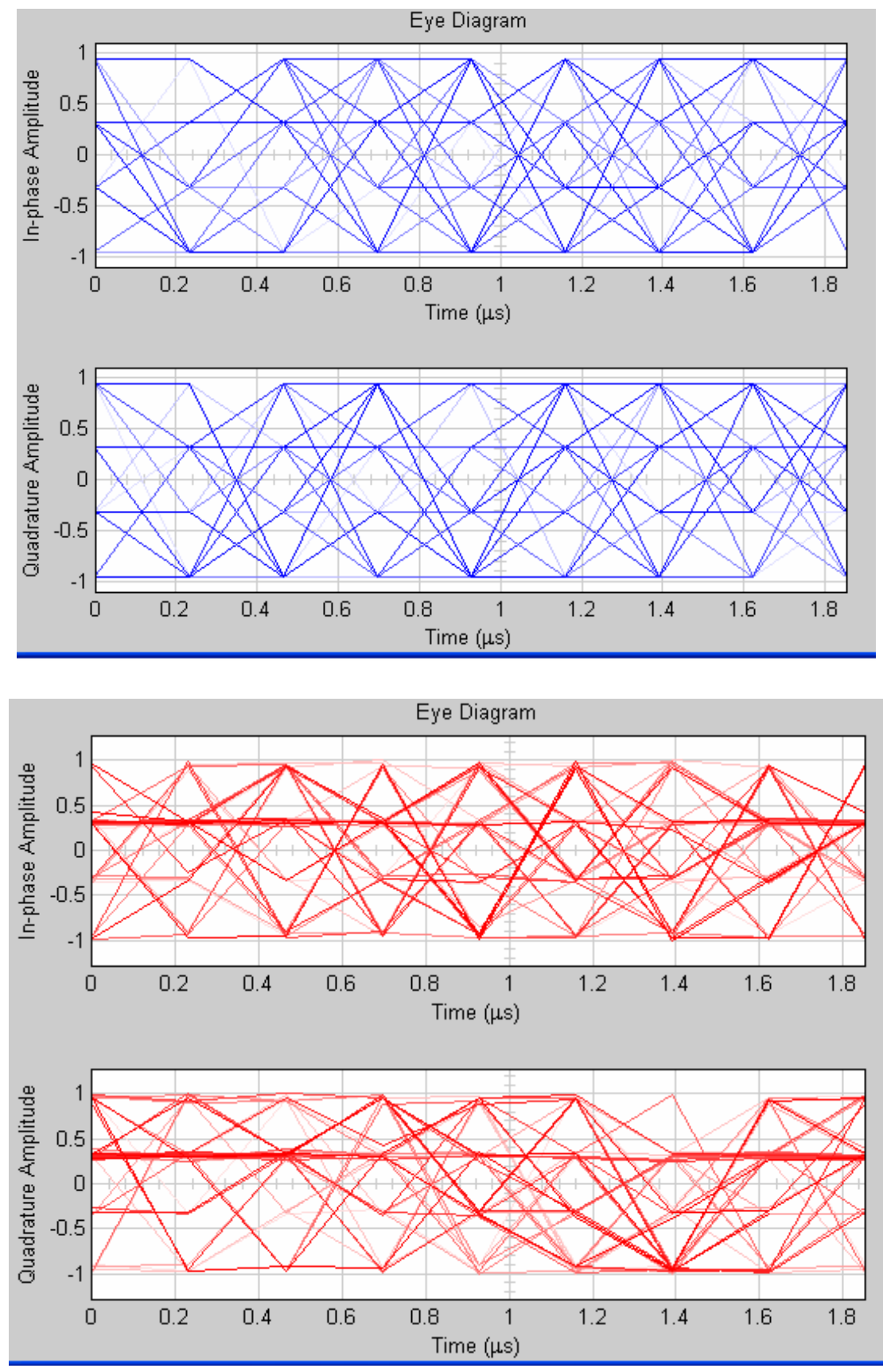

Fig. 5.12 16QAM $1 / 2$ Transmitted and Received Eye Diagrams BER $=3.94 e^{-6}$

\subsection{Summary}

In this chapter, the principles of a MIMO system were analyzed, in addition to how it can be used with OFDM modulation to obtain both frequency and space diversity in a 
terrestrial multipath Rayleigh channel. Toward this aim, an Alamouti encoder and decoder were employed, in conjunction with QPSK and 16QAM modulation schemes. From the simulation results, it is observed that MIMO, with two transmit and two receive antennas using OFDM and 16QAM, performs well in terms of received BER. The values shown in Tables 5.4 and 5.5 were obtained using MatLab version 7.6.0.324. 


\section{Chapter VI. An Approach to Cooperative Satellite Communications in 4G Mobile Systems}

\subsection{Cooperative Communications}

This chapter focuses upon the two main methods of Cooperative Communications, Decode and Forward and Amplify and Forward, and how they can be used in a new concept of Cooperative Satellite Communications. Both methods are analyzed in terms of symbol error rate and power allocation to determine which is more efficient, when relaying information from the satellite to a mobile node in the terrestrial network. Furthermore, the chapter presents a protocol that combines selective and incremental relaying to optimize the cooperative scheme.

Future 4G mobile systems will allow a subscriber to receive services anywhere and anytime at low costs. Such $4 \mathrm{G}$ systems will be capable of covering any geographical area by utilizing either terrestrial networks or satellite networks. To this aim, it is necessary to combine both networks into a hybrid architecture that possesses the flexibility to transmit high data rates from the source to the end user. In order to obtain such high data rates, it is also necessary to use higher order digital modulations, i.e., M-PSK or M-QAM, along with a bandwidth efficient scheme, like orthogonal frequency multiple access (OFDM) [4]. It is also imperative to adapt the recent trend of Cooperative Communications (CC) to this hybrid satellite/terrestrial network, so the link is as reliable as possible and the transmission of information is guaranteed.

$\mathrm{CC}$ works on the basis of a relay node that retransmits the signal to the destination node, and it combines two transmission phases. In Phase I, the source transmits a signal to both the relay node and the destination node; and in Phase II, the relay node 
retransmits the received signal to the destination node. Two methods are being used by CC, known as Decode and Forward (DF) and Amplify and Forward (AF). AF is simply an amplification of the signal by the relay node, and then, the amplified signal is transmitted. DF is a more complex approach, in which the relay node receives a signal, decodes and re-encodes it, and then transmits it to the destination node. CC can be categorized in fixed relay and adaptive relay schemes. Fixed relaying has the advantage of easy implementation, but it is not efficient in the bandwidth usage because half of the channel resources are allocated to the relay for transmission. This reduces the overall rate. Adaptive relaying includes selective and incremental relaying, and it is bandwidth efficient.

The case of satellite transmissions under consideration is one in which the satellite acts as the source node. A relay node is placed in areas where mobile users may lose their link to the satellite, thus requiring another way to relay the signal. Examples of this include a mobile user traveling and approaching places where the satellite link may be intermittent, or completely disrupted (tunnels, vegetation areas, buildings, etc.)

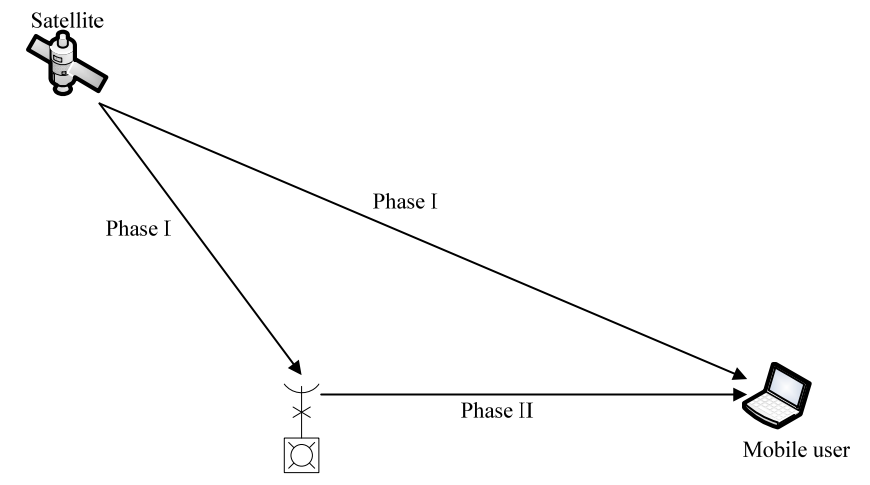

Fig. 6.1 Cooperative Satellite Communications Showing Phase I and Phase II

In Phase I, the received signal (y) at the relay and destination nodes is: 
$\mathrm{y}_{\mathrm{s}, \mathrm{d}}=\sqrt{\mathrm{P}} \mathrm{h}_{\mathrm{s}, \mathrm{d}} \cdot \mathrm{x}(\mathrm{t})+\mathrm{n}_{\mathrm{s}, \mathrm{d}}$ and $y_{s, r}=\sqrt{P} h_{s, r} \cdot x(t)+n_{s, r}$

$P$ is the transmitted power at the source, $x(t)$ is the transmitted information symbol, $n_{s, d}$ and $n_{s, r}$ are the additive noise in the source-destination $(\mathrm{s}, \mathrm{d})$ and source-relay $(\mathrm{s}, \mathrm{r})$ channels, and $\mathrm{h}_{\mathrm{s}, \mathrm{d}}$ and $\mathrm{h}_{\mathrm{s}, \mathrm{r}}$ are the channel coefficients for the s-d and s-r channels.

The channels are considered as zero-mean, complex Gaussian random variables, with variances of $\delta_{\mathrm{s}, \mathrm{d}}^{2}$ and $\delta_{\mathrm{s}, \mathrm{r}}^{2}$. The noise terms $\mathrm{n}_{\mathrm{s}, \mathrm{d}}$ and $\mathrm{n}_{\mathrm{s}, \mathrm{r}}$ are modeled as zero-mean complex Gaussian random variables, with variance No.

In Phase II, the relay sends a signal to the destination, based on what it receives from the source:

$\mathrm{y}_{\mathrm{r}, \mathrm{d}}=\mathrm{h}_{\mathrm{r}, \mathrm{d}} \cdot \kappa\left(\mathrm{y}_{\mathrm{s}, \mathrm{r}}\right)+\mathrm{n}_{\mathrm{r}, \mathrm{d}}$,

where r,d represents the link between the relay and the destination, and $\kappa$ varies depending on the type of scheme in use (AF or DF).

The destination receives two copies from the signal $\mathrm{x}(\mathrm{t})$ through the $\mathrm{s}, \mathrm{d}$ link and the $\mathrm{r}, \mathrm{d}$ link. It is necessary to combine both incoming signals at the destination. The optimal technique that triggers the best signal to noise ratio (SNR) is the maximal ratio combiner (MRC). At the MRC output, the obtained SNR is equal to the SNR from both the s,d and r,d links.

The outage probability $[13,14]$ is the probability that the mutual information is less than the rate $\mathrm{R}$, and in $\mathrm{AF}$, the outage probability is [1]:

$\mathrm{P}_{\mathrm{r}}\left[M I_{\mathrm{AF}}<\mathrm{R}\right] \approx\left(\frac{\delta_{\mathrm{s}, \mathrm{r}}^{2}+\delta_{\mathrm{r}, \mathrm{d}}^{2}}{2 \delta_{\mathrm{s}, \mathrm{d}}^{2}\left(\delta_{\mathrm{s}, \mathrm{r}}^{2} \delta_{\mathrm{r}, \mathrm{d}}^{2}\right)}\right) \cdot\left(\frac{2^{2 \mathrm{R}}-1}{P / N_{0}}\right)^{2}$ Achieving diversity 2 
$\mathrm{I}_{\mathrm{AF}}$ is the mutual information between the source and destination, while $\mathrm{R}$ is the rate. The same analysis can be extended to DF systems, resulting in an outage probability as follows:

$\mathrm{P}_{\mathrm{r}}\left[M I_{\mathrm{DF}}<\mathrm{R}\right] \approx \frac{1}{2 \delta_{\mathrm{s}, \mathrm{r}}^{2}} \cdot \frac{2^{2 \mathrm{R}}-1}{P / N_{0}} \quad$ Achieving diversity 1

\subsection{Adaptive Cooperation Schemes}

With fixed relaying, there is a $50 \%$ loss in the spectral efficiency due to transmission in two phases. The performance of DF is limited to the weakest source-relay and relaydestination link, reducing the diversity gain to one. Some other approaches [26] are aimed at resolving this limitation. They are known as selective relaying and incremental relaying and examined in further detail below.

\section{Selective Relaying}

In DF selective relaying (SDF), the relay node decodes and forwards the signal, only if its SNR is above a certain value, which is known as the threshold value [28, 29]. If the source-relay link suffers some fading or attenuations, making the SNR value less than the threshold, the relay will not decode and forward the information to the destination node.

When the received signal at the relay node is strong enough (SNR > Threshold), the SNR of the combined MRC signal at the destination is the sum of the received SNR from the source and relay, as stated above. In order for an outage event to occur, both the source-destination s,d and source-relay s,r channels should be in outage, or the combined source-destination and relay-destination channel should be in outage $[32,1]$, which results in a diversity of two. The outage expression is given by: 
$\mathrm{P}_{\mathrm{r}}\left[M I_{\mathrm{SDF}}<\mathrm{R}\right] \approx\left(\frac{\delta_{\mathrm{s}, \mathrm{r}}^{2}+\delta_{\mathrm{r}, \mathrm{d}}^{2}}{2 \delta_{\mathrm{s}, \mathrm{d}}^{2}\left(\delta_{\mathrm{s}, \mathrm{r}}^{2} \delta_{\mathrm{r}, \mathrm{d}}^{2}\right)}\right)$

This situation results in the same diversity gain as the AF case above. Thus, it can be concluded that with high SNR, selective relaying DF and AF have the same diversity gain.

Incremental Relaying

In this case, there is a feedback channel from the destination to the relay. The destination sends an acknowledgement message to the relay [33], if it correctly receives the signal sent by the source. If this occurs, the relay does not need to transmit in Phase II [27] (see Figure 6.2).

This scheme features the most optimal spectral efficiency among the above described approaches because the relay does not always need to transmit, and the Phase II transmission will depend on the channel characteristics in Phase I between the source and the destination.

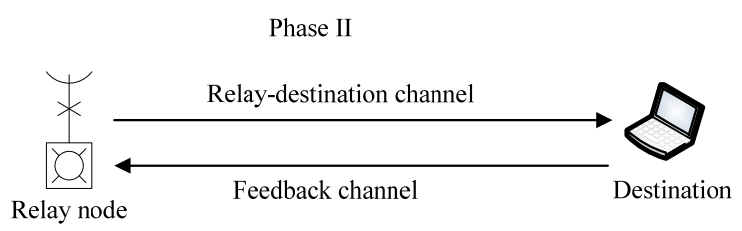

Fig. 6.2 Phase II Occurs Only If the Destination Node Asks the Relay Node to Forward This Information

If the transmission in Phase I from the source to the destination is successful, Phase II does not occur, and the source uses the next timeframe to transmit new data. On the other hand, if the Phase I transmission is unsuccessful, then Phase II must take place, and the 
relay will send information to the destination. This could occur when the mobile user loses the link to the satellite. The outage expression $[34,1]$ is given by:

$\mathrm{P}_{\mathrm{r}}\left[M I_{\mathrm{AF}}<\mathrm{R}\right] \approx\left(\frac{1}{2 \delta_{\mathrm{s}, \mathrm{d}}^{2}} \cdot \frac{\delta_{\mathrm{s}, \mathrm{r}}^{2}+\delta_{\mathrm{r}, \mathrm{d}}^{2}}{\delta_{\mathrm{s}, \mathrm{r}}^{2} \delta_{\mathrm{r}, \mathrm{d}}^{2}}\right) \cdot\left(\frac{2^{\overline{\mathrm{R}}}-1}{P / N_{0}}\right)^{2} \quad \overline{\mathrm{R}}=\frac{\mathrm{R}}{2} \cdot\left(1+\exp \left(-\frac{2^{\mathrm{R}}-1}{\mathrm{P} / \mathrm{N}_{0}}\right)\right)$

The performance degrades when the rate $\mathrm{R}$ increases, but it degrades faster for incremental relaying because of the inherent loss in the spectral efficiency [35]. For sufficiently high $\mathrm{R}$, direct transmission is more efficient than relaying. Incremental relaying performs better because it works at a much higher rate of spectral efficiency than the other relaying mechanisms, providing a diversity gain of two.

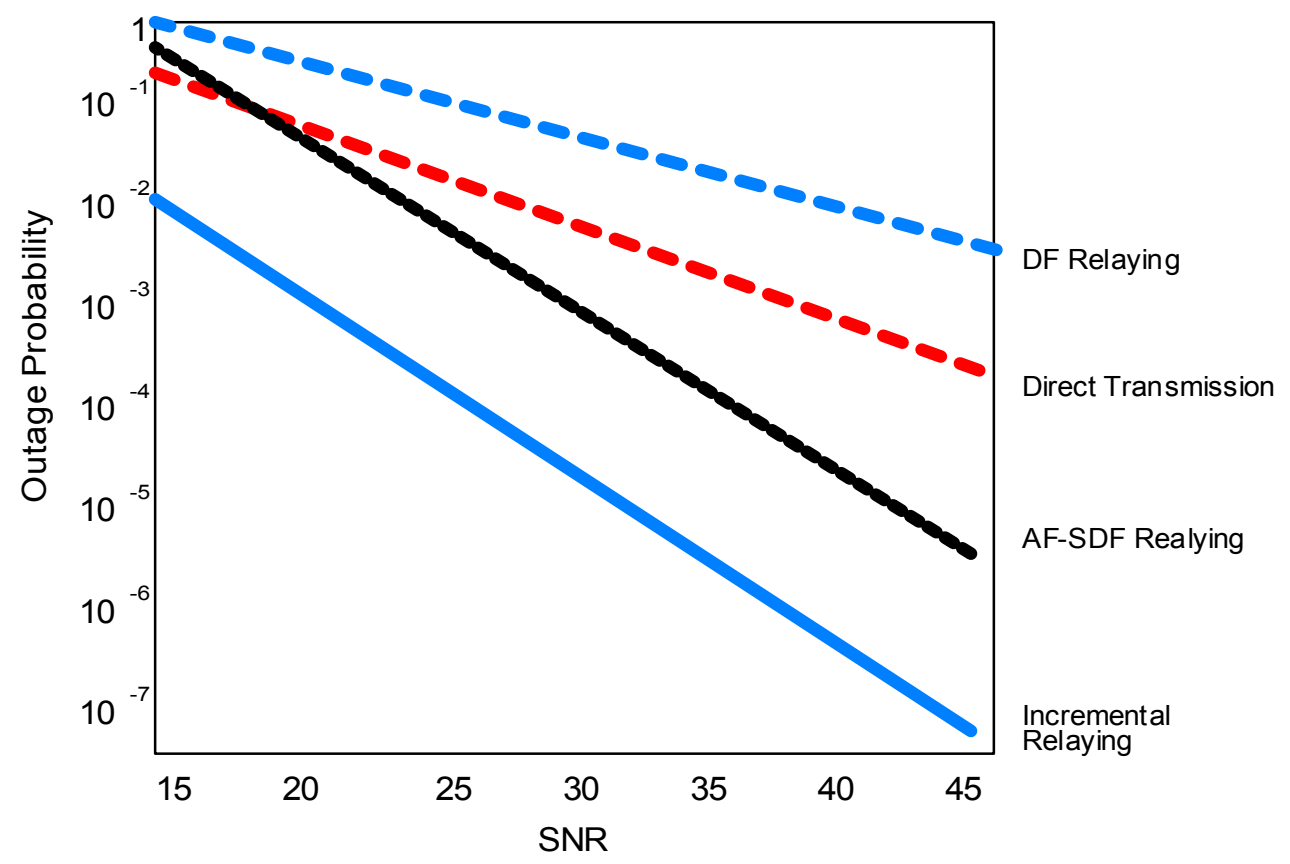

Fig. 6.3 Performance of Relaying Schemes versus SNR [dB] 


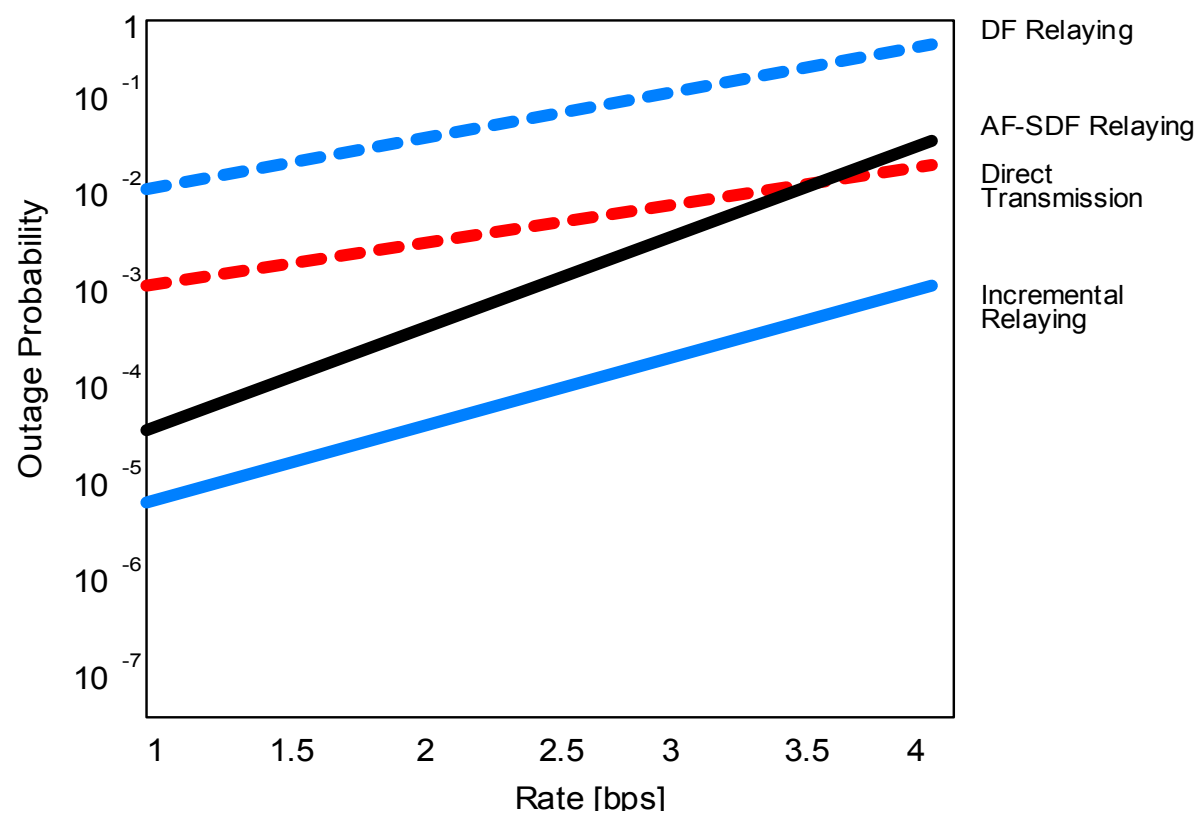

Fig. 6.4 Performance of Relaying Schemes versus Rate [bps]

Figures 6.3 and Fig. 6.4 show the performances of different schemes versus direct transmission, for the cases of outage probability versus SNR and outage probability versus rate. It is clear from both that incremental relaying allows for the best performance, while DF fixed relaying offers diversity of only one. In these graphs, $\delta_{\mathrm{s}, \mathrm{d}}^{2}=$ $1, \delta_{\mathrm{s}, \mathrm{r}}^{2}=\delta_{\mathrm{r}, \mathrm{d}}^{2}=0.5$, and noise variance is one.

\subsection{Symbol Error Rate Analyses of DF and AF}

DF SER Analysis

The analysis of symbol error rate was completed using DF [31] with 8PSK and 16QAM modulations. Future $4 \mathrm{G}$ systems will require high order modulations to guarantee that high data rates are delivered to the end user. These high data rates are needed by many applications, but especially by those that use multimedia, such as video and data, etc. 
Given the information Regarding the channel coefficients $h_{s, d}$ and $h_{r, d}$ between the source and destination and the relay and destination, and assuming that the transmitted symbol $x$ has average energy of 1 , the SNR of the MRC output is given by [6]:

$$
\mathrm{S} N R_{M R C}=\frac{\mathrm{Ps}\left|\mathrm{h}_{\mathrm{s}, \mathrm{d}}\right|^{2}+\mathrm{P}_{\mathrm{R}}\left|\mathrm{h}_{\mathrm{r}, \mathrm{d}}\right|^{2}}{\mathrm{~N}_{0}}
$$

SER formulations for both 8PSK and 16QAM are given by the equations:

$$
\begin{aligned}
& I_{\mathrm{PSK}}(\vartheta)=\frac{1}{\pi} \int_{0}^{(M-1) \pi / M} \exp \left(-\frac{\mathrm{b}_{\mathrm{PSK}} \vartheta}{\operatorname{Sin}^{2} \theta}\right) \mathrm{d} \theta \\
& I_{Q A M}(\vartheta)=3 Q\left(\sqrt{b_{Q A M} \vartheta}\right)-\frac{9}{4} Q^{2}\left(\sqrt{b_{Q A M} \vartheta}\right)
\end{aligned}
$$

$\vartheta$ is the $\mathrm{SNR}, \mathrm{b}_{\mathrm{PSK}}=\sin ^{2}(\Pi / 8), \mathrm{b}_{\mathrm{QAM}}=1 / 5$, and $\mathrm{Q}$ is the Gaussian function. If 8PSK is used in a DF cooperation system, with instantaneous SNR $I$, then the conditional SER of the system with channel coefficients $h_{s, d}, h_{s, r}, h_{r, d}$ can be expressed as:

$S E R_{P S K}=I_{P S K}\left(S N R_{M R C}\right)$

If 16QAM is used in the system, then the conditional SER [31] of such a system is given by the following expression:

$S E R_{Q A M}=I_{Q A M}\left(S N R_{M R C}\right)$

In the case of QPSK and 4QAM modulation, the conditional SER given by (6.9) and (6.10) is the same. This is because QPSK and 4QAM have the same constellations, so the detection of the phases involves the same complexity. In Phase II, if the relay node decodes the symbol correctly, it is forwarded to the destination with power $\mathrm{P}_{\mathrm{R}}^{\sim}=\mathrm{P}_{\mathrm{R}}$. If the symbol is not decoded correctly, however, it is not forwarded, and $\mathrm{P}_{\mathrm{R}}^{\sim}=0$. If 8PSK is used, the chances of incorrectly and correctly decoding at the relay are: 


$$
I_{\mathrm{PSK}}\left(\frac{\mathrm{Ps}\left|\mathrm{h}_{\mathrm{s}, \mathrm{r}}\right|^{2}}{\mathrm{~N}_{0}}\right) \text {, and } 1-I_{\mathrm{PSK}}\left(\frac{\mathrm{Ps}\left|\mathrm{h}_{\mathrm{s}, \mathrm{r}}\right|^{2}}{\mathrm{~N}_{0}}\right)
$$

If, on the other hand, 16QAM is used, the chances of incorrectly and correctly decoding a symbol at the relay are:

$$
I_{\mathrm{QAM}}\left(\frac{\mathrm{P}_{\mathrm{S}}\left|\mathrm{h}_{\mathrm{s}, \mathrm{r}}\right|^{2}}{\mathrm{~N}_{0}}\right) \text {, and } 1-I_{\mathrm{QAM}}\left(\frac{\mathrm{P}_{\mathrm{S}}\left|\mathrm{h}_{\mathrm{s}, \mathrm{r}}\right|^{2}}{\mathrm{~N}_{0}}\right)
$$

The link between the relay node and the destination node can be modeled as a Rayleigh fading channel because the path between them can be obstructed, and a direct line of sight may not exist. The SER for a DF cooperation scheme under a Rayleigh fading channel, using 8PSK modulation, can be expressed as follows [35]:

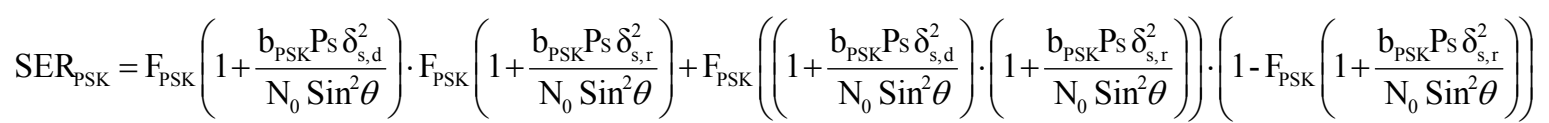

For a system using 16QAM over a Rayleigh fading channel with DF cooperation, the SER is expressed as:

$\operatorname{SER}_{\mathrm{QAM}}=\mathrm{F}_{\mathrm{QAM}}\left(1+\frac{\mathrm{b}_{\mathrm{QAM}} \mathrm{P}_{\mathrm{S}} \delta_{\mathrm{s}, \mathrm{d}}^{2}}{2 \mathrm{~N}_{0} \operatorname{Sin}^{2} \theta}\right) \cdot \mathrm{F}_{\mathrm{QAM}}\left(1+\frac{\mathrm{b}_{\mathrm{QMN}} \mathrm{PS}_{\mathrm{s}} \delta_{\mathrm{s}, \mathrm{r}}^{2}}{2 \mathrm{~N}_{0} \operatorname{Sin}^{2} \theta}\right)+\mathrm{F}_{\mathrm{QAM}}\left(\left(1+\frac{\mathrm{b}_{\mathrm{QAM}} \mathrm{P}_{\mathrm{S}} \delta_{\mathrm{s}}^{2}}{2 \mathrm{~N}_{0} \operatorname{Sin}^{2} \theta}\right) \cdot\left(1+\frac{\mathrm{b}_{\mathrm{QMN}} \mathrm{P}_{\mathrm{S}} \delta_{\mathrm{r}, \mathrm{d}}^{2}}{2 \mathrm{~N}_{0} \operatorname{Sin}^{2} \theta}\right)\right) \cdot\left(1-\mathrm{F}_{\mathrm{QAM}}\left(1+\frac{\mathrm{b}_{\mathrm{QMM}} \mathrm{P}_{\mathrm{S}} \delta_{\mathrm{s}, \mathrm{r}}^{2}}{2 \mathrm{~N}_{0} \operatorname{Sin}^{2} \theta}\right)\right)$

$\mathrm{F}_{\mathrm{PSK}}$ and $\mathrm{F}_{\mathrm{QAM}}$ depend on $\mathrm{x}(\theta)$.

\section{DF SER Approximation}

The SER of a DF cooperation [31] system using 8PSK and 16QAM modulations can be upper bounded as:

$$
\mathrm{SER}_{\mathrm{S}} \leq \frac{(\mathrm{M}-1) \mathrm{N}_{0}^{2}}{\mathrm{M}^{2}} \cdot \frac{\mathrm{M} \mathrm{b}_{\mathrm{S}} \delta_{\mathrm{s}, \mathrm{r}}^{2}+(\mathrm{M}-1) \mathrm{b} \mathrm{P}_{\mathrm{R}} \delta_{\mathrm{r}, \mathrm{d}}^{2}+(2 M-1) N_{0}}{\left(N_{0}+\mathrm{b} \mathrm{P}_{\mathrm{S}} \delta_{\mathrm{s}, \mathrm{d}}^{2}\right)+\left(N_{0}+\mathrm{b} \mathrm{P}_{\mathrm{S}} \delta_{\mathrm{s}, \mathrm{r}}^{2}\right)+\left(N_{0}+\mathrm{b} \mathrm{P}_{\mathrm{R}} \delta_{\mathrm{r}, \mathrm{d}}^{2}\right)},
$$


where $\mathrm{b}=\mathrm{b}_{\mathrm{PSK}}$ for $8 \mathrm{PSK}$ signals, and $\mathrm{b}=\mathrm{b}_{\mathrm{QAM}}$ for 16QAM signals; $\mathrm{M}=8$ in $8 \mathrm{PSK}$, and $\mathrm{M}=16$ in $16 \mathrm{QAM}$.

If $\delta^{2}{ }_{\mathrm{s}, \mathrm{d}} \neq 0, \delta_{\mathrm{s}, \mathrm{r}}^{2} \neq 0$, and $\delta_{\mathrm{r}, \mathrm{d}}^{2} \neq 0$, this indicates that all of the link channels $\left(\mathrm{h}_{\mathrm{s}, \mathrm{d}} \mathrm{h}_{\mathrm{s}, \mathrm{r}}\right.$ and $h_{r, d}$ ) are available. Then, $P_{S} / N_{0}$ and $P_{R} / N_{0}$ approach infinity, and the SER of the system using 8PSK and 16QAM modulations can be approximated as:

$\mathrm{SER}_{\mathrm{S}} \approx \frac{\mathrm{N}_{0}^{2}}{\mathrm{~b}^{2}} \cdot \frac{1}{\mathrm{P}_{\mathrm{S}} \delta_{\mathrm{s}, \mathrm{d}}^{2}}\left(\frac{A^{2}}{\mathrm{P}_{\mathrm{S}} \delta_{\mathrm{s}, \mathrm{r}}^{2}}+\frac{B^{2}}{\mathrm{P}_{\mathrm{R}} \delta_{\mathrm{r}, \mathrm{d}}^{2}}\right)$,

where $\mathrm{b}, \mathrm{A}$, and $\mathrm{B}$ depend on the type of modulation, whether it is 8PSK or 16QAM.

8PSK: $\mathrm{A}=0.494, \mathrm{~B}=0.377$

16QAM: $\mathrm{A}=0.64, \mathrm{~B}=0.53$

AF SER Approximation

An approximate expression for SER using AF can be obtained. If all the channels $\left(\mathrm{h}_{\mathrm{s}, \mathrm{d}}\right.$ $\mathrm{h}_{\mathrm{s}, \mathrm{r}}$ and $\mathrm{h}_{\mathrm{r}, \mathrm{d}}$ ) are available (meaning that $\delta_{\mathrm{s}, \mathrm{d}}^{2} \neq 0, \delta_{\mathrm{s}, \mathrm{r}}^{2} \neq 0$, and $\delta_{\mathrm{r}, \mathrm{d}}^{2} \neq 0$ ), then when $\mathrm{P}_{\mathrm{S}} / \mathrm{N}_{0}$ and $\mathrm{P}_{\mathrm{R}} / \mathrm{N}_{0}$ approach infinity, the SER of AF using 8PSK and 16QAM modulations is given by [30]:

$\mathrm{SER}_{\mathrm{S}} \leq \frac{\mathrm{A} \mathrm{N}_{0}^{2}}{\mathrm{~b}^{2}} \cdot \frac{P_{S} \delta^{2}{ }_{s, r}+P_{R} \delta^{2}{ }_{r, d}}{P^{2}{ }_{S} P_{R} \delta^{2}{ }_{s, d} \delta^{2}{ }_{s, r} \delta^{2}{ }_{r, d}}$,

where $\mathrm{b}$ and $\mathrm{A}$ depend on the type of modulation and are given by:

8PSK: $A=0.3742, b=b_{\text {PSK }}$

16QAM: $A=0.53, b=b_{Q A M}$ 
The following figures show both DF and AF SER graphs versus $\mathrm{P} / \mathrm{N}_{0}[\mathrm{~dB}]$. The three results exhibited below are the exact SER formulation, the upper bound formulation, and the asymptotically tight approximation, given that $\delta^{2}{ }_{\mathrm{s}, \mathrm{d}}=\delta_{\mathrm{s}, \mathrm{r}}=\delta_{\mathrm{r}, \mathrm{d}}^{2}=1$, and $\mathrm{N}_{0}=1$.

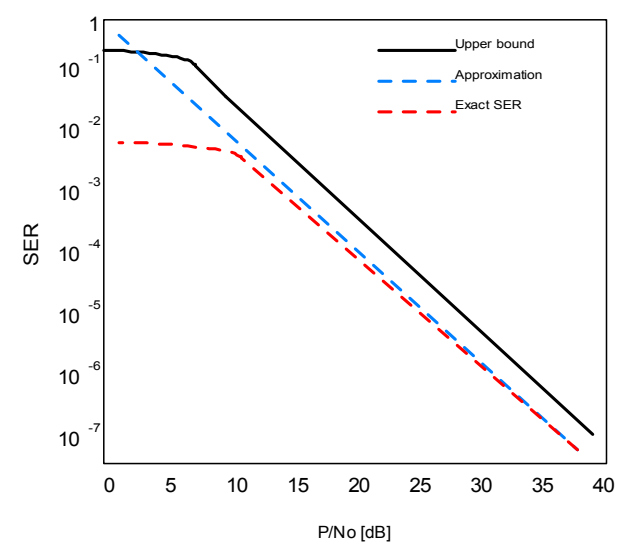

Fig. 6.5 DF Cooperative Communications System with QPSK

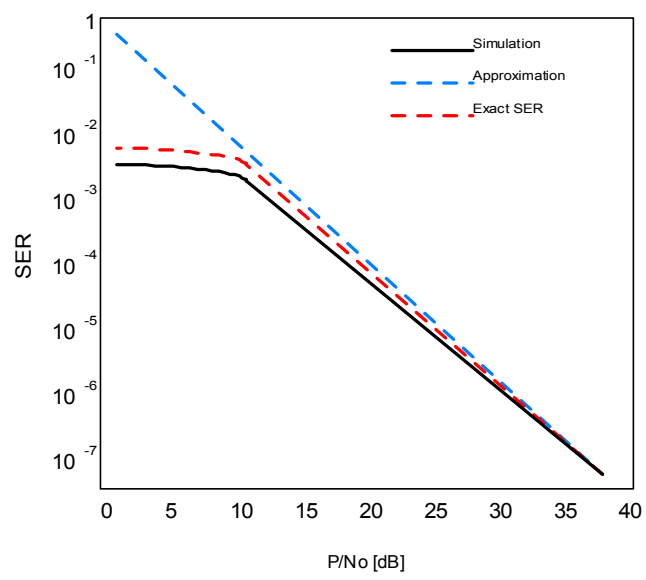

Fig. 6.6 AF Cooperative Communications System with QPSK

\subsection{Analysis of Power Distribution in DF Schemes}

In this section, the optimum power distribution, both at the source and the relay node [36], is obtained. Note that, as stated before, the power at the source is $\mathrm{P}_{\mathrm{S}}$, and the power at the relay is $\mathrm{P}_{\mathrm{R}}$. 
In a DF cooperation scheme using 8PSK and 16QAM modulations [31], if all the channels are available $\left(\mathrm{h}_{\mathrm{s}, \mathrm{d}} \mathrm{h}_{\mathrm{s}, \mathrm{r}}\right.$ and $\mathrm{h}_{\mathrm{r}, \mathrm{d}}$, and $\delta_{\mathrm{s}, \mathrm{d}}^{2} \neq 0, \delta_{\mathrm{s}, \mathrm{r}}^{2} \neq 0$, and $\delta_{\mathrm{r}, \mathrm{d}}^{2} \neq 0$ for high SNR and $P=P_{S}+P_{R}$, the power distribution [35] is:

$$
\begin{gathered}
\mathrm{P}_{\mathrm{S}}=\frac{\delta_{\mathrm{s}, \mathrm{r}}+\sqrt{\delta_{\mathrm{s}, \mathrm{r}}^{2}+8\left(A^{2} / B\right) \delta_{\mathrm{r}, \mathrm{d}}^{2}}}{3 \delta_{\mathrm{s}, \mathrm{r}}+\sqrt{\delta_{\mathrm{s}, \mathrm{r}}^{2}+8\left(A^{2} / B\right) \delta_{\mathrm{r}, \mathrm{d}}^{2}}} \cdot P \\
\mathrm{P}_{\mathrm{R}}=\frac{2 \delta_{\mathrm{s}, \mathrm{r}}}{3 \delta_{\mathrm{s}, \mathrm{r}}+\sqrt{\delta_{\mathrm{s}, \mathrm{r}}^{2}+8\left(A^{2} / B\right) \delta_{\mathrm{r}, \mathrm{d}}^{2}}} \cdot P
\end{gathered}
$$

A and B depend on the type of modulation, 8PSK or 16QAM, as stated in the previous section.

It is important to note that the expressions (6.18) and (6.19) do not depend on the source-destination channel; they only depend on the links between the source-relay and relay-destination. In addition, the optimum power ratio of the source power $\mathrm{P}_{\mathrm{S}}$ over the total power $\mathrm{P}$ is less than one and larger than $1 / 2$ [35]; on the other hand, the optimum ratio of $\mathrm{P}_{\mathrm{R}}$ at the relay over the total power $\mathrm{P}$ is greater than 0 and less than $1 / 2$ [31]. Thus, $1 / 2<P_{\mathrm{S}} / P<1$ and $0<P_{\mathrm{R}} / P<1$

indicating that more power should be added at the source, and less power at the relay. This consequence is significant to this case because the satellite is the source, and it must possess the greater power. If $\delta^{2} \mathrm{~s}, \mathrm{r}<<\delta^{2} \mathrm{r}$, d, link quality between the source-relay is less than that of the relay-destination. $\mathrm{P}_{\mathrm{S}}$ tends to $\mathrm{P}$, and $\mathrm{P}_{\mathrm{R}}$ tends to 0 , meaning that all the power at the source must be used, given that the link quality between the relaydestination is better. This is the appropriate case when the satellite link indicates strong fading due to rain or another atmospheric impairment. 
On the contrary, if $\delta^{2} \mathrm{~s}, \mathrm{r}>>\delta^{2} \mathrm{r}$,d, this means the source-relay channel is in much better condition than the relay-destination link. In this case, $P_{S}$ and $P_{R}$ approach $1 / 2$, and equal amounts of power should be allocated at both the source and relay. In the satellite link case, since the satellite power cannot be increased, the power must be incremented at the relay each time the relay-destination link fades considerably.

It is important to note that the relay-destination link is modeled as a Rayleigh fading channel, in which there is no direct line of sight between the relay and destination, thus resulting in strong fading.

In order to obtain diversity two, the source-relay and relay-destination links should be appropriately balanced. If the source-relay link is unavailable, it is difficult for the relay to perform its task of decoding and forwarding the received symbol.

Therefore, the forwarding task of the relay is less important, and it is more logical to inject increased power at the source. On the contrary, if the source-relay channel quality is very strong, the relay can decode the signal from the source. In this case, the relay can be considered as a copy of the source; the same amount of power can be injected in both. The amount of power also depends on the constellation size; for high order constellations, such as 8PSK or 16QAM, the amount of power must be greater than in the case of QPSK or 4QAM.

Below, the discussion considers three cases of power allocation using D [2]: (1) relaydestination channel is unavailable $\left(\delta_{\mathrm{r}, \mathrm{d}}^{2}=0\right) ;(2)$ source-relay channel is unavailable $\left(\delta_{\mathrm{s}, \mathrm{r}}^{2}\right.$ $=0$ ); and (3) source-destination channel is unavailable $\left(\delta^{2} \mathrm{~s}, \mathrm{~d}=0\right)$.

Case 1. If the relay-destination channel is unavailable, from (6.13), the SER of a DF cooperation system using 8PSK modulation can be expressed by: 
$\mathrm{SER}_{\mathrm{PSK}}=\mathrm{F}_{\mathrm{PSK}}\left(1+\frac{\mathrm{b}_{\mathrm{PSK}} \mathrm{P}_{\mathrm{S}} \delta_{\mathrm{s}, \mathrm{d}}^{2}}{\mathrm{~N}_{0} \operatorname{Sin}^{2} \theta}\right) \leq \frac{\mathrm{A} \mathrm{N}_{0}}{\mathrm{~b}_{\mathrm{PSK}} \mathrm{Ps}_{\mathrm{s}, \mathrm{d}}^{2}}$,

where $\mathrm{F}_{\mathrm{PSK}}$ and $\mathrm{A}$ are defined above for the 8PSK case.

Extrapolating from (6.14), a similar equation for the case of 16QAM when the relaydestination link is unavailable can be formed:

$\mathrm{SER}_{\mathrm{QAM}}=\mathrm{F}_{\mathrm{QAM}}\left(1+\frac{\mathrm{b}_{\mathrm{QAM}} \mathrm{P}_{\mathrm{S}} \delta_{\mathrm{s}, \mathrm{d}}^{2}}{2 \mathrm{~N}_{0} \operatorname{Sin}^{2} \theta}\right) \leq \frac{2 \mathrm{AN}_{0}}{\mathrm{~b}_{\mathrm{QAM}} \mathrm{P}_{\mathrm{S}} \delta_{\mathrm{s}, \mathrm{d}}^{2}}$,

where $\mathrm{F}_{\mathrm{QAM}}$ and $\mathrm{A}$ are specified above for the 16QAM case.

From the above equations (6.20) and (6.21), the optimum power distribution is $\mathrm{P}_{\mathrm{S}}=\mathrm{P}$ and $\mathrm{P}_{\mathrm{R}}=0$. As expected, if there is no relay-destination link, the sole option is to use direct transmission between the source and destination, allocating all the power at the source.

Case 2. If the source-relay channel is unavailable, from (6.13) and (6.14), the SER of a DF cooperation system, using either modulation, is expressed as follows:

$\operatorname{SER}_{\mathrm{P}} \leq \frac{2 \mathrm{~A} \mathrm{~N}_{0}}{\mathrm{bP}_{\mathrm{S}} \delta_{\mathrm{s}, \mathrm{d}}^{2}}$

A will depend on whether the system uses $8 \mathrm{PSK}$ or 16QAM, and $\mathrm{b}=\mathrm{b}_{\mathrm{PSK}}$ for $8 \mathrm{PSK}$, and $b=b_{\mathrm{QAM}} / 2$ for 16QAM. In this case, the optimum power distribution is $\mathrm{P}_{\mathrm{S}}=\mathrm{P}$ and $\mathrm{P}_{\mathrm{R}}$ $=0$.

Case 3. If the source-destination channel is unavailable (causing Phase II transmission, see Section VI) from (6.13) and (6.14), the SER of a DF cooperation system with 8PSK or 16QAM is given by: 
$\mathrm{SER}_{\mathrm{S}}=\mathrm{F}_{\mathrm{i}}\left(1+\frac{\mathrm{b} \mathrm{P}_{\mathrm{S}} \delta_{\mathrm{s}, \mathrm{r}}^{2}}{\mathrm{~N}_{0} \operatorname{Sin}^{2} \theta}\right)+\mathrm{F}_{\mathrm{i}}\left(1+\frac{\mathrm{b} \mathrm{P}_{\mathrm{R}} \delta_{\mathrm{r}, \mathrm{d}}^{2}}{\mathrm{~N}_{0} \operatorname{Sin}^{2} \theta}\right) \cdot\left(1-\mathrm{F}_{\mathrm{i}}\left(1+\frac{\mathrm{b} \mathrm{P}_{\mathrm{S} 1} \delta_{\mathrm{s}, \mathrm{r}}^{2}}{\mathrm{~N}_{0} \operatorname{Sin}^{2} \theta}\right)\right)$,

where $\mathrm{i}=1$ and $\mathrm{b}=\mathrm{b}_{\mathrm{PSK}}$ for $8 \mathrm{PASK}$, and $\mathrm{I}=2$ and $\mathrm{b}=\mathrm{b}_{\mathrm{QAM}} / 2$ for $16 \mathrm{QAM}$. If the source-relay and relay-destination are available, the SER in (6.23) can be approximated as:

$\mathrm{SER}_{\mathrm{S}} \approx \frac{A N_{0}^{2}}{b^{2}}\left(\frac{1}{\mathrm{P}_{\mathrm{S}} \delta_{\mathrm{s}, \mathrm{r}}^{2}}+\frac{1}{\mathrm{P}_{\mathrm{R}} \delta_{\mathrm{r}, \mathrm{d}}^{2}}\right)$

Here, $b=b_{\text {PSK }}$ for 8 PSK, and $b=b_{\text {QAM }} / 2$ for 16QAM. A also depends on the type of modulation, as expressed above [10].

In this last case, the power distribution for both 8PSK and 16QAM is:

$$
\mathrm{P}_{\mathrm{S}}=\frac{\delta_{\mathrm{r}, \mathrm{d}}}{\delta_{\mathrm{s}, \mathrm{r}}+\delta_{\mathrm{r}, \mathrm{d}}} \cdot P \quad \mathrm{P}_{\mathrm{R}}=\frac{\delta_{\mathrm{s}, \mathrm{r}}}{\delta_{\mathrm{s}, \mathrm{r}}+\delta_{\mathrm{r}, \mathrm{d}}} \cdot P
$$

When the source-destination channel is unavailable, the system is modeled as a two hop system. This conclusion is important in the case that the satellite loses the link with the mobile user and needs to use the relay node to transfer the service. The mobile node may have entered a zone out of the satellite's reach and will depend on the relay node to receive the signal (see Figure 6.7).

The power at the satellite is dependent upon the channel quality between the relay and destination, the channel quality between the satellite itself and the relay, and the overall power $\mathrm{P}$. 


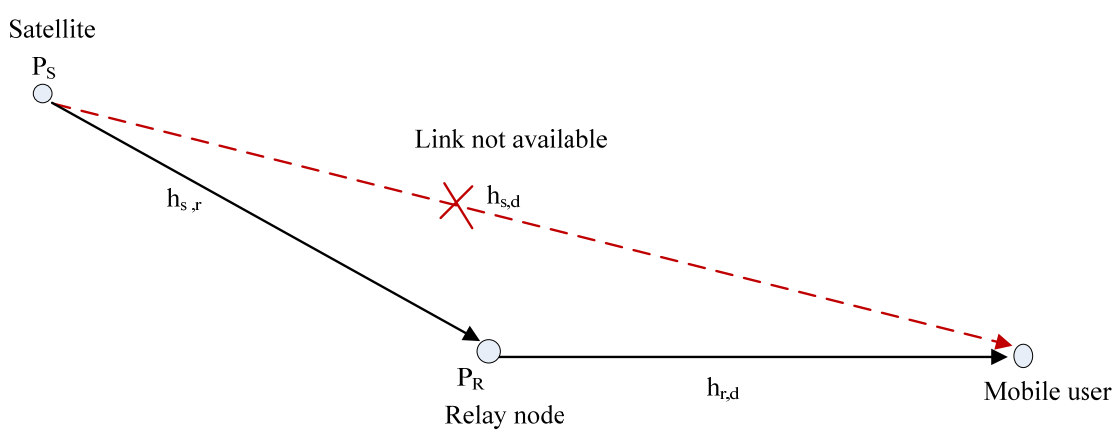

Fig. 6.7 Case 3 - No Source-Destination Link

The optimum power distribution for an AF system using either an 8PSK or 16QAM modulation can be expressed as [30]:

$$
\begin{gathered}
\mathrm{P}_{\mathrm{S}}=\frac{\delta_{\mathrm{s}, \mathrm{r}}+\sqrt{\delta_{\mathrm{s}, \mathrm{r}}^{2}+8 \delta_{\mathrm{r}, \mathrm{d}}^{2}}}{3 \delta_{\mathrm{s}, \mathrm{r}}+\sqrt{\delta_{\mathrm{s}, \mathrm{r}}^{2}+8 \delta_{\mathrm{r}, \mathrm{d}}^{2}}} \cdot P \\
\mathrm{P}_{\mathrm{R}}=\frac{2 \delta_{\mathrm{s}, \mathrm{r}}}{3 \delta_{\mathrm{s}, \mathrm{r}}+\sqrt{\delta_{\mathrm{s}, \mathrm{r}}^{2}+8 \delta_{\mathrm{r}, \mathrm{d}}^{2}}} \cdot P
\end{gathered}
$$

From (6.25) and (6.26), it can be deduced that the optimum power distribution in an AF system does not depend on the type of modulation used. This differs from the DF scheme, in which the optimum power distribution does in fact depend on the type of modulation. This is because in the AF case, the relay receives, amplifies and forwards the signal, regardless of the type of modulation.

In DF, the relay uses the modulation type in order to decode and re-encode the data, which explains why the power distribution in such a scheme depends on the modulation. In addition, the above equations (6.25) and (6.26) reveal that the optimum ratio of $\mathrm{P}_{\mathrm{S}}$ to the overall power $\mathrm{P}$ is less than 1 and larger than $1 / 2$, and the ratio of power $\mathrm{P}_{\mathrm{R}}$ to the overall power is larger than 0 and less than $1 / 2$. 


\subsection{DF and AF Performance}

Decode and Forward

For high SNR, the SER performance of a DF system is given by (6.16). Substituting the optimum power distribution from equations (6.18) and (6.19) in (6.16), the following is the result [2]:

$\operatorname{SER}_{\mathrm{S}} \approx D_{D F}^{-2}(\Phi)^{-2}$

Below is the SER of a DF scheme, in which $D_{D F}^{-2}$ is defined by equation (6.28) for cases of 8PSK and 16QAM.

$$
D_{\mathrm{DF}}=\frac{2 \sqrt{2} \mathrm{~b} \delta_{\mathrm{s}, \mathrm{d}} \delta_{\mathrm{s}, \mathrm{r}} \delta_{\mathrm{r}, \mathrm{d}}}{\sqrt{\mathrm{B}}} \frac{\left(\delta_{\mathrm{s}, \mathrm{r}}+\sqrt{\delta_{\mathrm{s}, \mathrm{r}}^{2}+8\left(\mathrm{~A}^{2} / \mathrm{B}\right) \delta_{\mathrm{r}, \mathrm{d}}^{2}}\right)^{1 / 2}}{\left(3 \delta_{\mathrm{s}, \mathrm{r}}+\sqrt{\delta_{\mathrm{s}, \mathrm{r}}^{2}+8\left(\mathrm{~A}^{2} / \mathrm{B}\right) \delta_{\mathrm{r}, \mathrm{d}}^{2}}\right)^{3 / 2}},
$$

where $\mathrm{b}=\mathrm{b}_{\mathrm{PSK}}$ for $8 \mathrm{PSK}$, and $\mathrm{b}=\mathrm{b}_{\mathrm{QAM}} / 2$ for 16QAM, and $\Phi=P / N_{0}$.

An analysis of equation (6.27) indicates that adaptive DF cooperation results in a diversity of 2, depending only on the characteristics of the channel links. Equation (6.28) is known as the cooperation gain of a DF system and provides information regarding the optimal performance gain that can be obtained using DF Cooperation. If the channel between the source and relay is worse than the channel between the relay and destination, the cooperation gain can be reduced to:

$D_{\mathrm{DF}}=\frac{\mathrm{b} \delta_{\mathrm{s}, \mathrm{d}} \delta_{\mathrm{s}, \mathrm{r}}}{\mathrm{A}}$

On the other hand, if the channel between the source and relay is much better than the channel quality between the relay and destination, the cooperation gain can be reduced to:

$D_{\mathrm{DF}}=\frac{\mathrm{b} \delta_{\mathrm{s}, \mathrm{d}} \delta_{\mathrm{r}, \mathrm{d}}}{2 \sqrt{\mathrm{B}}}$ 


\section{Amplify and Forward}

A similar analysis can be performed for the Amplify and Forward scheme. The SER is presented in equation (6.17), and combining this SER with equations (6.25) and (6.26) yields [10]:

$$
\begin{aligned}
& \operatorname{SER}_{\mathrm{S}} \approx D_{A F}^{-2}(\Phi)^{-2} \\
& D_{\mathrm{AF}}=\frac{2 \sqrt{2} \mathrm{~b} \delta_{\mathrm{s}, \mathrm{d}} \delta_{\mathrm{s}, \mathrm{r}} \delta_{\mathrm{r}, \mathrm{d}}}{\sqrt{\mathrm{B}}} \frac{\left(\delta_{\mathrm{s}, \mathrm{r}}+\sqrt{\delta_{\mathrm{s}, \mathrm{r}}^{2}+8 \delta_{\mathrm{r}, \mathrm{d}}^{2}}\right)^{1 / 2}}{\left(3 \delta_{\mathrm{s}, \mathrm{r}}+\sqrt{\delta_{\mathrm{s}, \mathrm{r}}^{2}+8 \delta_{\mathrm{r}, \mathrm{d}}^{2}}\right)^{3 / 2}}
\end{aligned}
$$

Equation (6.32) is the cooperation gain of an $\mathrm{AF}$ scheme and determines the best performance of a system using AF. Equation (6.31) shows that AF also results in a diversity of order 2, which is the same gain obtained from an adaptive DF cooperation system.

Comparing the cooperation gain of DF to that of AF results in the ratio $\beta$, which is given by $\beta=D_{\mathrm{DF}} / D_{\mathrm{AF}}$

The three possible cases of channel quality can be analyzed as follows: (1) sourcerelay channel is worse than the relay-destination channel $\left(\delta_{\mathrm{s}, \mathrm{r}}^{2}<<\delta_{\mathrm{r}, \mathrm{d}}^{2}\right)$,

$$
\beta \approx \frac{\sqrt{B}}{A}>1 \quad \text {, and DF performs better than AF; (2) source-relay channel is better }
$$

than the relay-destination channel $\left(\delta_{\mathrm{s}, \mathrm{r}}^{2}>>\delta_{\mathrm{r}, \mathrm{d}}^{2}\right), \beta \approx 1$, and DF and AF perform equally; and (3) source-relay channel is equal to the relay-destination channel $\left(\delta_{\mathrm{s}, \mathrm{r}}^{2}=\delta_{\mathrm{r}, \mathrm{d}}^{2}\right)$

$$
\beta=\sqrt{\frac{1+\sqrt{1+8 A^{2} / B}}{4}}\left(\sqrt{\left(\frac{6}{3+\sqrt{1+8 A^{2} / B}}\right)}\right)^{3} .
$$


Inserting the values of $\mathrm{A}$ and $\mathrm{B}$ for $8 \mathrm{PSK}$ and 16QAM yields $\beta \approx 1.0670$ for $8 \mathrm{PSK}$, and for $16 \mathrm{QAM}, \beta \approx 1.0378$.

\subsection{Selective and Incremental Relaying in Satellite/Terrestrial Cooperation}

From Section 6.5, it is apparent that DF performs better in two of the three cases, and performs similarly to AF when the source-relay channel is better than the relaydestination channel. The use of higher order modulations over satellite links [4] must be carefully designed, and strong error correction algorithms must be used. In addition, as mentioned at the beginning of this dissertation, OFDM is required to obtain a better spectral efficiency and to transmit high data rates. This raises the consideration (in most cases) that the source-relay channel may be worse than the relay-destination channel.

It is important to note that although the relay-destination channel is modeled as a Rayleigh multipath, which is a type of channel with strong fading, the use of OFDM and higher modulation order are more reliable here than in the satellite-relay channel.

In satellite links, OFDM depends on increased time diversity, and high order modulations depend on pre-distortion in order to function successfully. In this case, since the cooperation gain $\beta>1$, the Decode and Forward scheme performs better than the Amplify and Forward scheme. By combining DF with selective and incremental relaying, an even stronger scheme can be developed.

As shown in Figure 8, the destination node requests transmission from the relay node only if its SNR is less than the threshold value, meaning that the signal is not strong enough to obtain the data sent by the source. If the destination node loses the satellite link for some reason, the SNR drops below the threshold and requests Phase II from the relay 
node. The relay node then employs selective relaying and transmits the decoded signal to the destination node, only if its own SNR is above the threshold value.

Thus, for the relay node to transmit, two conditions must be met: (1) the destination node must request transmission; and (2) the SNR on the relay itself must be above threshold. If the SNR of the relay node is below the threshold value, this indicates the signal at the relay node is not strong or reliable enough to initiate a relay transmission to the destination node.

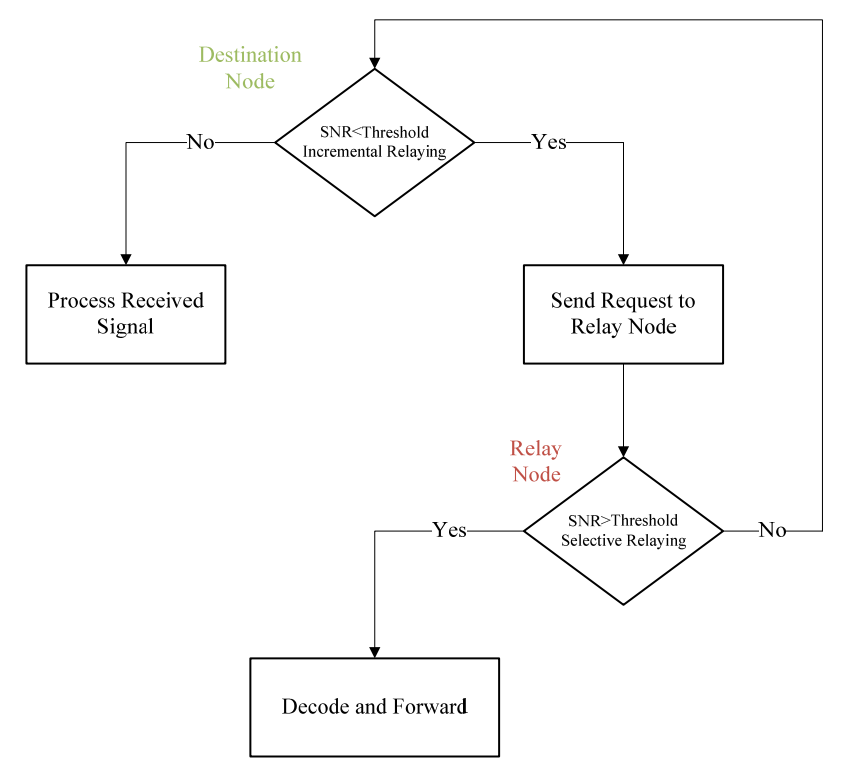

Fig. 6.8 Incremental and Selective Relaying

The relay node will remain idle if it does not receive a request from the destination node, and/or if its own SNR is low. If the relay node does not receive the request, it will remain idle even if its SNR is high.

This is the main difference between this proposed scheme and selective relaying, in which the relay decodes and forwards the signal as long as its SNR is greater than the threshold value. The difference between the proposed scheme and incremental relaying is 
that when the destination requests a transmission from the relay, it will occur if the SNR in the relay is high. In this case, the destination completely loses the signal if both channels (satellite-destination and satellite- relay) are unavailable, as shown in Figure 6.9.

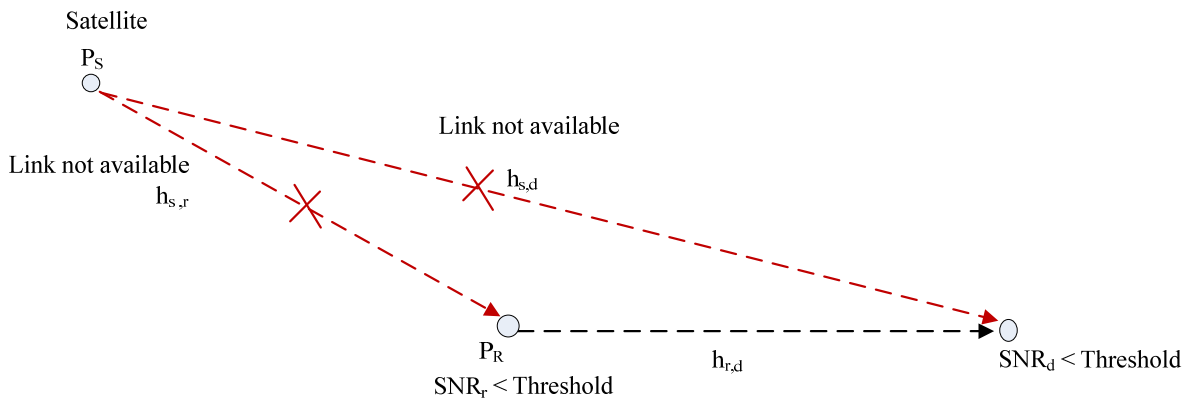

Fig. 6.9 Both SNRs (at Destination and Relay) Are Low

\subsection{Simulation Results}

As mentioned before, Phase II occurs when the destination node requests this, and SNR at the relay is above a certain value. A simulation of Phase II consists of the relay node using OFDM 8PSK and 16QAM, a Rayleigh multipath fading channel, and the destination node where the SER is measured.

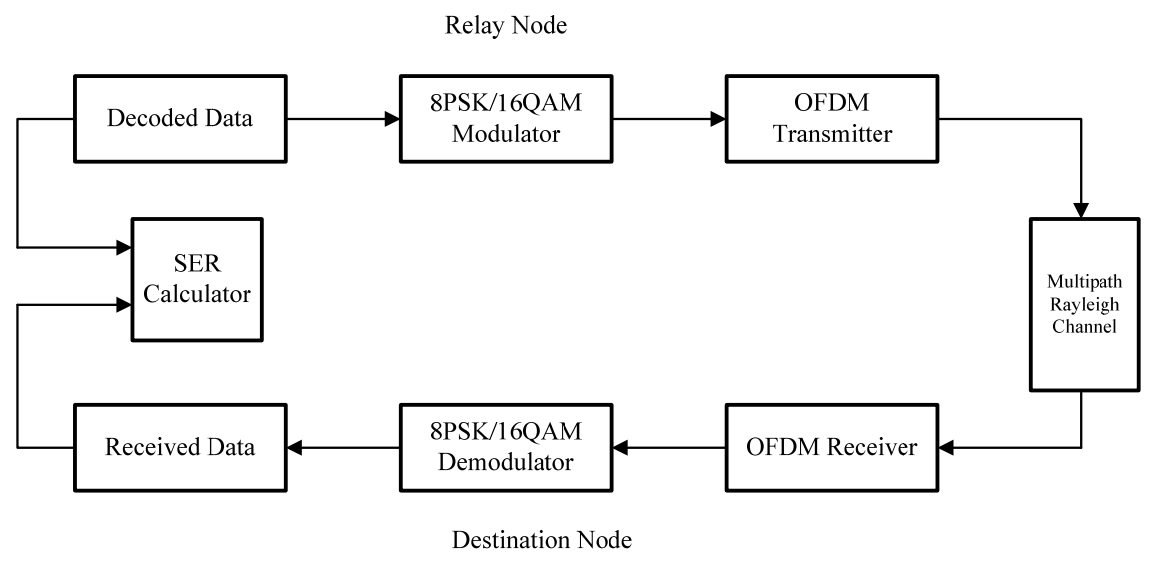

Fig. 6.10 Phase II Physical Layer 
The signal spectrum at the output of the relay node and at the input of the destination node shows how the Rayleigh channel affects the overall frequency distribution. By using a strong error correction method, these impairments can be overcome, and the resulting SER (6.13) is within the limits of performance. Figure 6.11 shows the signal spectrum and the eye diagram of the transmitted and received signals.

The effects of the Rayleigh multipath over the frequency response can be seen at the destination node (in red). The OFDM signal is attenuated at different frequency components. Since the OFDM signal is composed of several individual carriers, this uneven attenuation effect is not as destructive as in a single carrier modulation. The individual carriers are therefore detected over a small bandwidth.
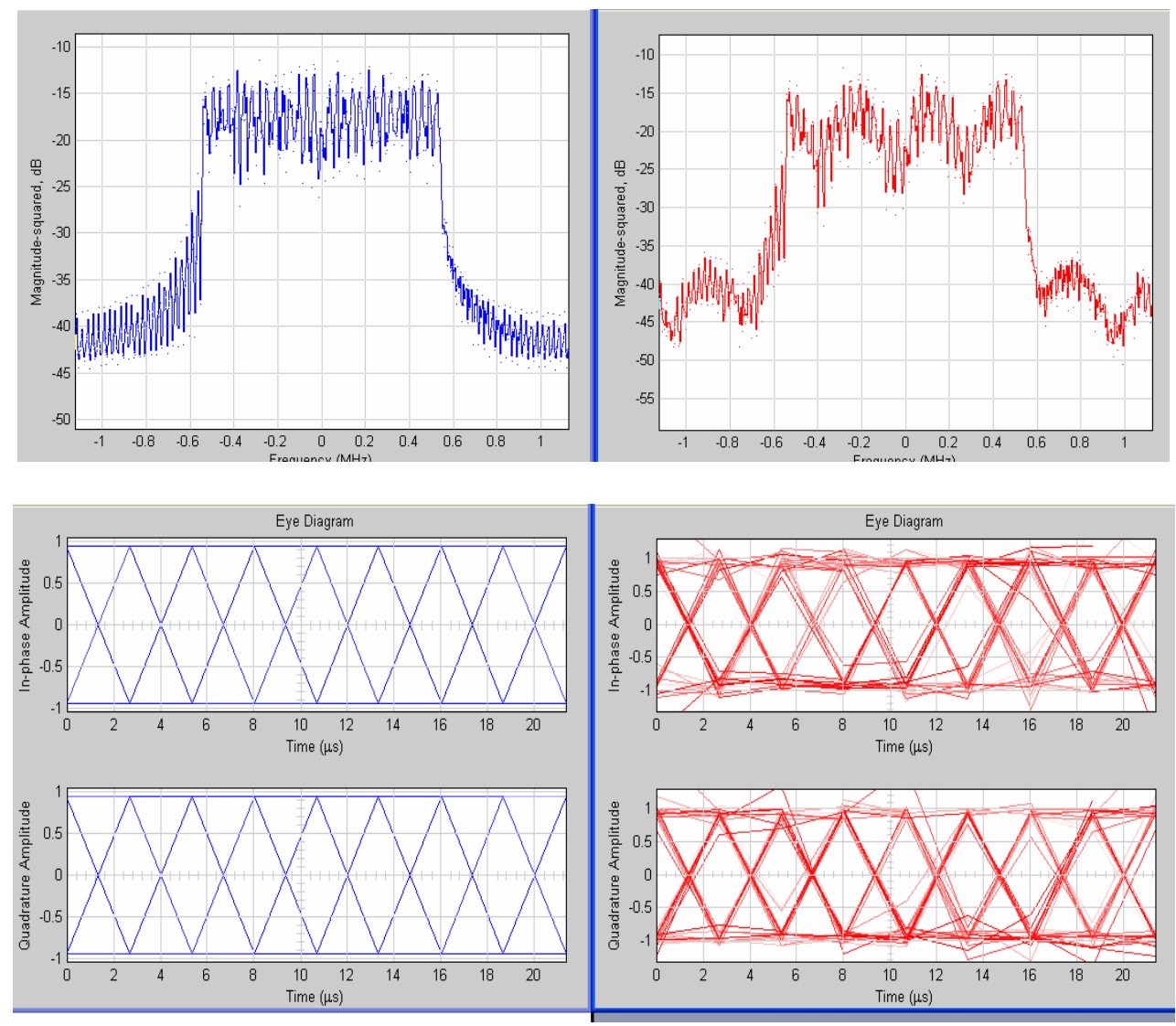

Fig. 6.11 OFDM Spectrum and Eye Diagram in Phase II Eb/No $=10 \mathrm{~dB}$ 
The simulation results for SER depend on the Eb/No value and the error correction employed, as shown in Figure 6.12.
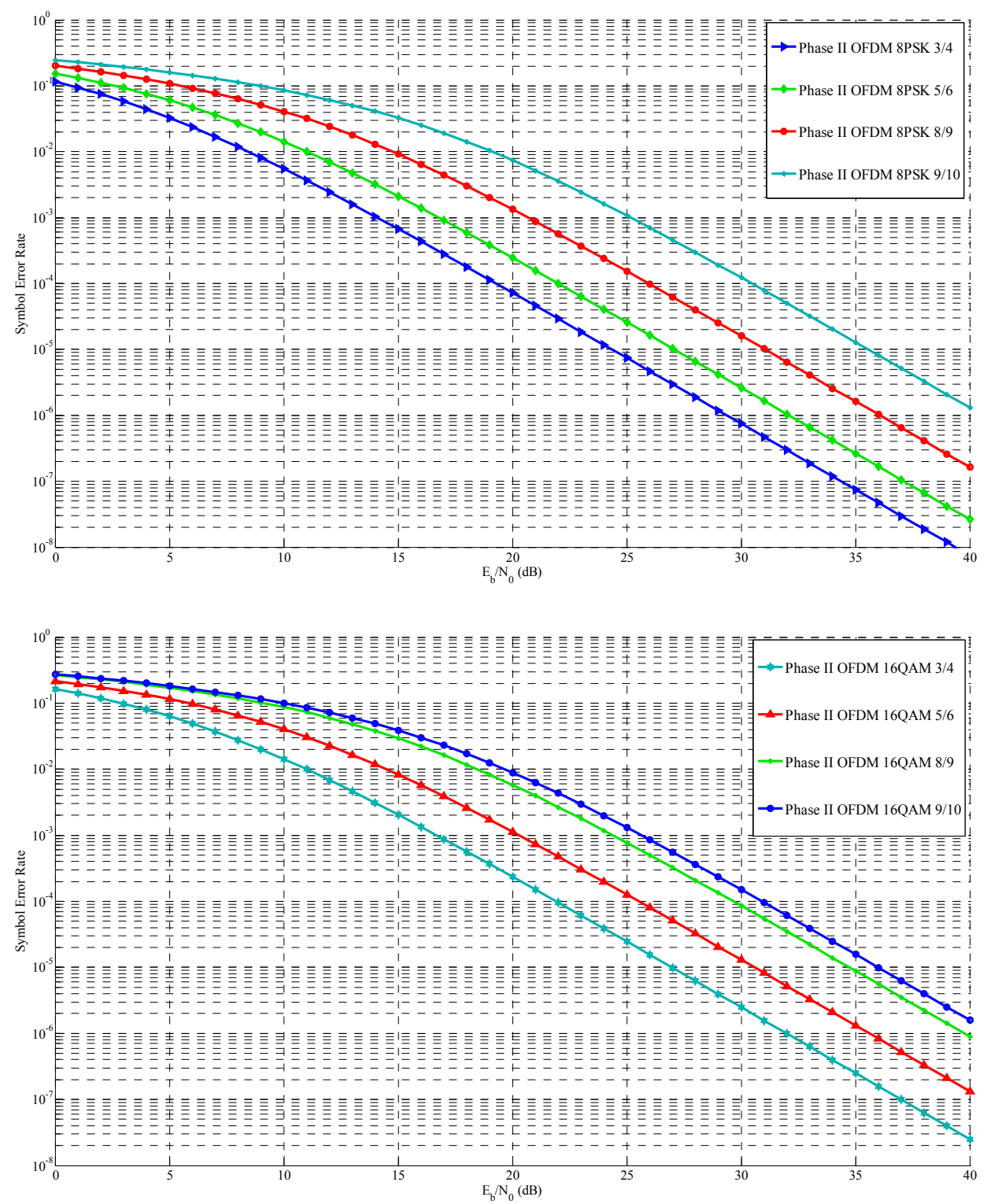

Fig. 6.12 Eb/No versus SER OFDM 8PSK/16QAM Phase II

\subsection{Summary}

Cooperative Satellite Communications will play a significant role in future 4G systems. The constant transfer of information from the satellite to the mobile unite must 
be guaranteed, even when the mobile unit travels to areas that are unreachable by the satellite. The Decode and Forward scheme appears to be the optimal option for the cooperating protocol, since it is never outperformed by the Amplify and Forward alternative. It is important to note here that even $\mathrm{AF}$ is easier to implement than $\mathrm{DF}$. AF does not allow the flexibility of adapting to bandwidth constraints that may be present when transferring the signal from the satellite link to the terrestrial link. OFDM and high modulation techniques, such as 8PSK and 16QAM, are needed in both channels. When the satellite-destination channel is not available, the power distribution will depend on the channel characteristics between the satellite-relay and the relay-destination.

To allow better bandwidth efficiency, the combination of selective and incremental relaying is the best option. As stated in Section VI, the transmission from the relay to the mobile user will take place only when the mobile user does not receive the signal from the satellite, and the signal at the relay node is sufficiently strong. 


\section{Chapter VII. Conclusions}

This study offers a solution to OFDM-based air interfaces to be used in 4G mobile systems. The aim of this dissertation was to find a solution to OFDM over satellite channels, which will be an important part of a system that tries to cover any user at any location and any time. Since $4 \mathrm{G}$ systems must accommodate high data rate transfers and high reliability, satellite communications will play an important role. Section 7.1 covers the findings of the dissertation, while Section 7.2 provides information regarding potential future work in this field.

\subsection{Dissertation Summary}

The first task is to formulate a model for a hybrid satellite terrestrial channel that can cover almost any geographical area and carries the necessary signal to transfer information to any user. To this aim, the satellite channel must feature a Rician distribution, while the terrestrial channel must have a Rayleigh distribution. The Rician distribution is selected because the terrestrial nodes are assumed to be fixed, and this guarantees a direct LOS between the earth node and the satellite. The terrestrial channel covers an area between the fixed earth station and a mobile user, who may be located anywhere, so it is impossible to ensure a LOS between the earth node and the mobile user. For this reason, a Rayleigh multipath fading channel is selected. Thus, the problem of using OFDM over satellite channels with long delays can be solved. A time diversity technique is proposed because it eliminates inter-symbol and inter-carrier interference. Each OFDM symbol is transmitted more than once, and the time between retransmission 
will be higher than the channel coherence time. This technique combines both the time diversity of the repetition code and the frequency diversity that OFDM has.

In the terrestrial link, MIMO techniques and Alamouti encoding and decoding are recommended. This is combined with an OFDM interface that uses high order modulation techniques, such as 16QAM. The number of transmitting and receiving antennas is 2, so the MIMO system will be a 2 X2 system. The issue of Cooperative Satellite Communications is solved through a new algorithm that forwards the received data from the fixed node to the mobile node. This algorithm is very efficient because it does not allow unnecessary transmissions and is based on SNR measures.

The table below presents a performance comparison between the existing single carrier satellite modulation techniques data rate versus a multiple carrier (OFDM) scheme using time diversity data rate.

Table 7.1 Performance of OFDM Time Diversity in Satellite Channels

\begin{tabular}{|l|c|c|c|c|}
\hline Modulation & $\begin{array}{c}\text { Bandwidth } \\
(\mathrm{MHz})\end{array}$ & FEC & $\begin{array}{c}\text { Single carrier data } \\
\text { rate. DVB S } \\
\text { scheme. (Mbps) }\end{array}$ & $\begin{array}{c}\text { Proposed OFDM } \\
\text { time diversity } \\
\text { scheme. (Mbps) }\end{array}$ \\
\hline QPSK & 5 & $1 / 2$ & 3.5 & 4.5 \\
\hline QPSK & 5 & $3 / 4$ & 5.3 & 6.75 \\
\hline QPSK & 5 & $5 / 6$ & 5.9 & 7.5 \\
\hline 16QAM & 5 & $1 / 2$ & $\mathrm{n} / \mathrm{a}$ & 9.01 \\
\hline 16QAM & 5 & $3 / 4$ & 10.6 & 13.5 \\
\hline 16QAM & 5 & $5 / 6$ & 12.4 & 15.1 \\
\hline
\end{tabular}




\subsection{Future Work}

Most of the work in this study is performed on the physical layer, so there remain many issues to be solved on this layer and on upper layers as well. With regard to the use of OFDM, additional problems must be addressed, including the high peak to average power ratio (PAPR). The selection of strong error correction algorithms is another issue that requires further study, as this will contribute to better BER values at the receiver and improve the amount of data transferred. Turbo codes offer this necessary flexibility, and its use with OFDM should be further explored.

The main limitation of OFDM-based transmissions systems is the high PAPR of the transmitted signals, and large peaks occasionally reach the amplifier saturation region, resulting in signal distortion. Several PAPR reductions schemes have been proposed and investigated; of these, the partial transmit sequence (PTS) scheme is an efficient approach and features less distortions for PAPR reduction by optimally combining signal subblocks. Selective mapping (SLM) is also a good approach, in which some statistically independent sequences are generated from the same information, and the sequence with the lowest PAPR is transmitted. Although these approaches have improved the OFDM scheme, further research is needed.

Upper layer challenges also remain under investigation to further improve the capacity and reliability of $4 \mathrm{G}$ systems. These include the network infrastructure and QoS support. Current QoS designs are aimed at a particular wireless system. However, providing QoS to only one system does not guaranteed end-to-end QoS because systems outside this umbrella scheme will also be involved in $4 \mathrm{G}$ solutions. Under the network infrastructure of $4 \mathrm{G}$ wireless environments, a problem arises when integrating non-IP and 
IP-based systems. Security concerns also need to be carefully designed, as their main problem with regard to $4 \mathrm{G}$ is flexibility. Since current security mechanisms are designed for specific purposes, such as voice, they are not applicable to $4 \mathrm{G}$ systems, which will be composed of various heterogeneous systems. Moreover, the key sizes and encryption and decryption algorithms of current designs are also fixed.

In general, the challenges to be addressed and more extensively studied are grouped into three categories: mobile stations, systems, and services. Some of these topics have received much attention from researchers, such as multimode user terminals, wireless system discovery, terminal mobility and QoS support. Others, however, require more study and include wireless system selection, security, failure, and survivability. Much research should be completed regarding the migration to $4 \mathrm{G}$ systems; furthermore, current systems must be implemented with an aim to ease the transition. Without these changes and infrastructure, $4 \mathrm{G}$ mobile systems will not be easily launched. 


\section{REFERENCES}

[1]. Savo G. Glisic, "Advanced Wireless Communications: 4G Cognitive and Cooperative Broadband Technology," Second Edition, Wiley: 2007. ISBN 978-0470-05977-7.

[2]. K. J. Ray Liu, Ahmed K. Sadek, Weifeng Su, and Andres Kwasinski, "Cooperative Communications and Networks," Cambridge: 2009. ISBN 978-0521-89513-2.

[3]. J. N. Patel and U. D. Dalal, "A Comparative Performance Analysis of OFDM Using MATLAB Simulation with M-PSK and M-QAM Mapping," Conference on Computational Intelligence and Multimedia Applications, International Conference on Volume 4, Dec. 13-15, 2007, pp. 406 - 410.

[4]. A. Vanelli-Coralli, G. E. Corazza, G. K. Karagiannidis, P.T. Mathiopoulos, D. S. Michalopoulos, C. Mosquera, S. Papaharalabos, and S. Scalise, "Satellite Communications: Research Trends and Open Issues," Satellite and Space Communications, International Workshop, September 13-14, 2007, pp. 71 - 75.

[5]. Hongwei Yang, "A road to future broadband wireless access: MIMO-OFDMBased air interface," Communications Magazine, IEEE Volume 43, Issue 1, Jan. 2005 , pp. $53-60$.

[6]. Suk Yu Hui and Kai Hau Yeung, "Challenges in the migration to 4G mobile systems," Communications Magazine, IEEE Volume 41, Issue 12, Dec. 2003, pp. $54-59$.

[7]. W. A. C. Fernando and R. M. A. P. Rajatheva, "Suitability of turbo coded OFDM for LEO satellite channels in global mobile communications," Consumer Electronics, 2001, ICCE, International Conference, 2001, pp. 196 - 197.

[8]. E. Karami, and M. Juntti, "A Near Optimum Low Complexity Detection Algorithm for MIMO OFDM Channels," Personal, Indoor and Mobile Radio Communications, 2007, PIMRC 2007, IEEE $18^{\text {th }}$ International Symposium, Sept. 3-7, 2007, pp. $1-5$.

[9]. A. Doufexi, S. Armour, A. Nix, and M. Beach, "Design considerations and initial physical layer performance results for a space time coded OFDM 4G cellular network," Personal, Indoor and Mobile Radio Communications, 2002, The $13^{\text {th }}$ IEEE International Symposium, Volume 1, Sept. 15-18, 2002, pp. $192-196$.

[10]. Yiqing Zhou, Jiangzhou Wang, and M. Sawahashi, "Downlink transmission of broadband OFCDM systems-part III: turbo-coded," Selected Areas in Communications, IEEE Journal, Volume 24, Issue 1, Jan. 2006, pp. 132 - 140. 
[11]. Simon Plass, Armin Dammann, Gerd Richter, and Martin Bossert, "Channel Correlation Properties in OFDM by using Time-Varying Cyclic Delay Diversity," Journal of Communications, Vol. 3, No. 3, July 2008.

[12]. Oh-Soon Shin, A. M. Chan, H. T. Kung, and V. Tarokh, "Design of an OFDM Cooperative Space-Time Diversity System," IEEE Transactions on Vehicular Technology, Vol. 56, No. 4, July 2007.

[13]. Kyungchun Lee and Lajos Hanzo, "MIMO-assisted hard versus soft decodingand-forwarding for network coding aided relaying systems," Wireless Communications, IEEE Transactions on Volume 8 , Issue 1, Jan. 2009, pp. 376 385 .

[14]. M. R. Souryal and B. R. Vojcic, "Performance of Amplify-and-Forward and Decode-and-Forward Relaying in Rayleigh Fading with Turbo Codes," Acoustics, Speech and Signal Processing, 2006, ICASSP 2006 Proceedings, IEEE International Conference on Volume 4, May 14-19, 2006, p. 4.

[15]. Yiftach Eisenberg and Cedric Logan, "Cooperative communications for improved throughput, range and covertness," Military Communications Conference, 2008, MILCOM 2008, IEEE Nov. 16-19, 2008, pp. 1 - 7.

[16]. Yi Wu and Matthias Patzold, "Performance analysis of amplify-and-forward cooperative communication systems with channel estimation errors," Communication Systems, 2008, ICCS 2008, $11^{\text {th }}$ IEEE Singapore International Conference, Nov. 19-21, 2008, pp. 1620-1624.

[17]. Feng Tian, Xiang-Gen Xia, and P. C. Ching, "Signal Detection for SpaceFrequency Coded Cooperative Communication System with Multiple Carrier Frequency Offsets," Wireless Communications and Networking Conference, 2007, WCNC, March 11-15, 2007, pp. 1221 - 1225.

[18]. A. A. Tahat, "Multi-User Channel Estimation in a 4G OFDM System," Personal, Indoor and Mobile Radio Communications, 2007, IEEE $18^{\text {th }}$ International Symposium, Sept. 3-7, 2007, pp. $1-5$.

[19]. A. Gotta and P. Barsocchi, "Experimental video broadcasting in DVB-RCS/S2 with land mobile satellite channel: A reliability issue," Satellite and Space Communications, 2008, IWSSC 2008, IEEE International Workshop, Oct. 1-3, 2008, pp. $234-238$.

[20]. L. Husson, J. C. Dany, S. Chambon, K. Berradi, and A. Beffani, "Modeling of mobile satellite channels by scalable clustering algorithm," Vehicular Technology Conference, 2002, IEEE 55 ${ }^{\text {th }}$ Volume 4, 2002, pp. 2004 - 2007. 
[21]. A. Abdi, W. C. Lau, M. S. Alouini, and M. Kaveh, "A new simple model for land mobile satellite channels: first- and second-order statistics," Wireless Communications, IEEE Transactions on Volume 2, Issue 3, May 2003, pp. 519 528.

[22]. S. Scalise, H. Ernst, and G. Harles, "Measurement and Modeling of the Land Mobile Satellite Channel at Ku-Band," Vehicular Technology, IEEE Transactions on Volume 57, Issue 2, March 2008, pp. 693 - 703.

[23]. A. Mehrnia and H. Hashemi, "Mobile satellite propagation channel. Part II-a new model and its performance," Vehicular Technology Conference, 1999, IEEE VTS 50th Volume 5, 1999, pp. 2780 - 2784.

[24]. M. Neri, P. Salmi, and G. E. Corazza, "Fractional Predistortion Techniques with Robust Modulation Schemes for Fixed and Mobile Broadcasting," $13^{\text {th }}$ IST Mobile and Wireless Communications Summit, 2004.

[25]. " $3{ }^{\text {rd }}$ Generation Partnership Project; Technical Specifications Group Radio Access Network; Evolved Universal Terrestrial Radio Access (E-UTRA)," Physical Channels and Modulation (Release 8), 3GPP TS 36.211 V8.1.0, 2007.

[26]. V. Mahinthan, H. Rutagemwa, J. W. Mark, and Xuemin Shen, "Performance of Adaptive Relaying Schemes in Cooperative Diversity Systems with ARQ," Global Telecommunications Conference, 2007, IEEE, Nov. 2007, pp. 4402 4406.

[27]. S. Ikki and M. H. Ahmed, "Performance Analysis of Incremental Relaying Cooperative Diversity Networks over Rayleigh Fading Channels," Wireless Communications and Networking Conference, 2008, April 2008, pp. 1311 1315 .

[28]. Bo Gui, Lin Dai, and L. J. Cimini, "Selective Relaying in Cooperative OFDM Systems: Two-Hop Random Network," Wireless Communications and Networking Conference, 2008, April 2008, pp. 996 - 1001.

[29]. F. A. Onat, A. Adinoyi, Yijia Fan, H. Yanikomeroglu, J. S. Thompson, and I. D. Marsland, "Threshold Selection for SNR-based Selective Digital Relaying in Cooperative Wireless Networks," Wireless Communications, IEEE Transactions on Volume 7, Issue 11, Part 1, November 2008, pp. 4226 - 4237.

[30]. Yan Wang and Fei Lin, "SER Performance Analysis and Power Allocation for Amplify-and-Forward Relay Networks," Wireless Communications, Networking and Mobile Computing, 2007, International Conference, Sept. 2007, pp. 1274 1277. 
[31]. Weifeng Su, A. K. Sadek, and K. J. R. Liu, "SER performance analysis and optimum power allocation for decode-and-forward cooperation protocol in wireless networks," Wireless Communications and Networking Conference, IEEE Volume 2, March 2005, pp. 984-989.

[32]. J. N. Laneman, D. N. C. Tse, and G. W. Wornell, "Cooperative diversity in wireless networks: Efficient protocols and outage behavior," Information Theory, IEEE Transactions on Volume 50, Issue 12, Dec. 2004, pp. 3062 - 3080.

[33]. P. Tarasak, H. Minn, and Yong Hoon Lee, "Analysis of incremental relaying protocol with RCPC in cooperative diversity systems," Vehicular Technology Conference, 2005, IEEE $62^{\text {nd }}$ Volume 4, Sept. 2005, pp. $2537-2541$.

[34]. I. Y. Abualhaol and M. M. Matalgah, "Outage Probability Analysis in a Cooperative UAVs Network over Nakagami-m Fading Channels," Vehicular Technology Conference, 2006, IEEE 64 ${ }^{\text {th }}$, Sept. 2006, pp. $1-4$.

[35]. Su Weifeng, A. K. Sadek, and K. J. R. Liu, "Cooperative Communications in wireless networks: performance analysis and optimum power allocation," Wireless Personal Communications, 44, Jan. 2008, pp. 181-217.

[36]. A. Reznik, S. R. Kulkarni, and S. Verdu, "Degraded Gaussian multirelay channel: capacity and optimal power allocation," Information Theory, IEEE Transactions on Volume 50, Issue 12, Dec. 2004, pp. $3037-3046$.

[37]. A. Sendonaris, E. Erkip, and B. Aazhang, "User cooperation diversity. Part I. System description," Communications IEE Transaction on Volume 51, Issue $\underline{11}$, Nov. 2003, pp. $1927-1938$.

[38]. A. Sendonaris, E. Erkip, and B. Aazhang, "User cooperation diversity. Part II. Implementation aspects and performance analysis," Communications ,IEEE Transactions on Volume 51, Issue 11, Nov. 2003, pp. $1939-1948$.

[39]. J. Sala and H. Durney, "Coarse time delay estimation for pre-correction of high power amplifiers in OFDM communications," Vehicular Technology Conference, 2002, IEEE $56^{\text {th }}$ Volume 4, 2002, pp. 2313 - 2317.

[40]. A. Azizzadeh and L. Mohammadi, "Degradation of BER by Group Delay in Digital Phase Modulation," Telecommunications, 2008, Fourth Advanced International Conference, June 8-13, 2008, pp. 350-354.

[41]. P. Savvopoulos and T. Antonakopoulos, "A DVB-S2 Signal Generator with Forward Satellite Channel Impairments," Instrumentation and Measurement Technology Conference, 2006, Proceedings of the IEEE, April 24-27, 2006, pp. $1833-1838$. 
[42]. S. Moriyama, K. Tsuchida, K., and M. Sasaki, "Digital transmission of high bit rate signals using 16DAPSK-OFDM modulation scheme," Broadcasting, IEEE Transactions, Vol. 44, Issue 1, March 1998, pp. 115-122.

[43]. A. Yazdan-Panah, B. Nosrat-Makouei, and R. G. Vaughan, "OFDM with CyclicPilot Time Diversity," Vehicular Technology Conference, 2007, IEEE $66^{\text {th }}$, Sept. 30 - Oct. 3, 2007, pp. 1371-1375.

[44]. K. M. Udofia and I.P. E. Otung, "Evaluating Time Diversity Performance on an On-board Processing Satellite to Earth Station Downlink," Next Generation Mobile Applications, Services and Technologies, 2008, $2^{\text {nd }}$ International Conference, Sept 16-19, 2008, pp. 325-330.

[45]. I. S. Raad and Xiaojing Huang, "Exploiting Time Diversity to Improve Block Spread OFDM in a Multipath Environment," Information and Communication Technologies, Volume 2, 2006, pp. 2444-2449.

[46]. C. Martin, A. Geurtz, and B. Ottersten, "Statistical Analysis and Optimal Design for Efficient Mobile Satellite Broadcast With Diversity," Vehicular Technology, IEEE Transactions on Vol. 57, Issue 2, March 2008, pp. 986-1000.

[47]. N. Miki, H. Atarashi, and M. Sawahashi, "Effect of time diversity in hybrid ARQ considering space and path diversity for VSF-OFCDM downlink broadband wireless access," Personal, Indoor and Mobile Radio Communications, 2004, $15^{\text {th }}$ IEEE International Symposium on Volume 1, Sept. 5-8, 2004, pp. $604-$ 608 .

[48]. Shaoping Chen and Cuitao Zhu, "ICI and ISI analysis and mitigation for OFDM systems with insufficient cyclic prefix in time-varying channels," Consumer Electronics, IEEE Transactions on Vol. 50, Issue 1, Feb. 2004, pp. 78-83.

[49]. R. S. Sherratt and R. Yang, "A Dual QPSK Soft-demapper for ECMA-368 Exploiting Time-Domain Spreading and Guard Interval Diversity," Consumer Electronics, 2007, Digest of Technical Papers, International Conference on Jan. 10-14, 2007, pp. 1-2.

[50]. Defeng Huang, K. B. Letaief, K.B. and Jianhua Lu, "Bit-interleaved timefrequency coded modulation for OFDM systems over time-varying channels," Communications, IEEE Transactions on Vol. 53, Issue 7, July 2005, pp. 11911199.

[51]. Gregory D. Durbing, "Space-Time Wireless Channels," Prentice Hall Communications, Engineering and Emerging Technologies Series, ISSN: 0-13065647-X. 2004. 
[52]. Wenzhen Li, Choi Look Law, V. K. Dubey, and J. T. Ong, "Ka-band land mobile satellite channel model incorporating weather effects," Communications Letters, IEEE Vol. 5, Issue 5, May 2001, pp. 194-196.

[53]. M. Rice, J. Slack, and B. Humphreys, "K-Band land mobile satellite channel characterization,” Int. J. Satellite Commun., Vol. 14, 1996, pp. 283-296.

[54]. E. Kubista, F. Perez Fontan. M. Angeles Vazquez Castro, S. Bunomo, B. R. Arbesser-Rasburg, and J.P.V. Poiares Baptista, "Ka-band propagation measurements and statistics for land mobile satellite applications," IEEE Trans. Veh. Technol., Vol. 49, May 2000, pp. 973-983.

[55]. Y. Xie and Y. Fang, "A general statistical channel model for mobile satellite systems," IEEE Trans. Veh. Technol., Vol. 49, May 2000, pp. 744-752.

[56]. C. Loo and J. S. Butterworth, "Land mobile satellite channel measurements and modeling", Proc. IEEE, Vol. 86, July 1998, pp. 1442-1463.

[57]. E. Lutz, D. Cyagn, M. Dippold, F. Dolainsky, and W. Papke, "The land mobile satellite communication channel-Recording, statistics and channel model," IEEE Trans. Veh. Technol. Vol. 40, May 1991, pp. 375-384.

[58]. T. S. Rappaport, "Wireless Communications: Principles and Practice," New Jersey: Prentice Hall, Inc., 1996.

[59]. W. C. Jakes, "Microwave Mobile Communications," Piscataway, New Jersey: IEEE Press, 1993.

[60]. Jose G. Ruiz, Beatriz Soret, M. Aguayo-Torres, M. Carmen, and J. Entrambasaguas, "On finite state Markov chains for Rayleigh channel modeling," Wireless Communication, Vehicular Technology, Information Theory and Aerospace \& Electronic Systems Technology, 2009, ${ }^{\text {st }}$ International Conference, May 17-20, 2009, pp. 191-195.

[61]. M. T. Islam, M. W. Numan, and N. Misran, "Design and implementation of Alamouti encoder for 4G wireless system." EUROCON, 2009, IEEE, May 18-23, 2009, pp. $1676-1680$.

[62]. J Pacheco, A. F. Herrero and J. C. Quiros, "Design and Implementation of a Hardware Module for MIMO Decoding in a 4G Wireless Receiver," VLSI Design, Vol. 2008, Article ID 312614, Hindawi Publishing Corporation, 2008, pp. 1-8.

[63]. V. Tarokh, H. Jafarkhani, and A. R. Calderbank, "Space time block codes from orthogonal designs," IEEE Transaction on Information Theory, Vol. 45, No. 5, July 1999, pp. 1456-1467. 
[64]. J. C. Guey, M. P. Fitz, M. R. Bell, and W. Y. Kuo. "Signal design for transmitter diversity wireless communications systems over Rayleigh fading channels," IEEE Transactions on Communications, 47:527-537, April 1999.

[65]. V. Tarokh, N. Seshadri, and A. R. Calderbank, "Space time block codes for high data rate wireless communications: performance criterion and code construction," IEEE Transaction on Information Theory, Vol. 44, No. 2, 1998, pp. 744-765.

[66]. A. Shokrollahi, B. Hassibi, B. M. Hochwall, and W. Sweldens, "Representation theory for high rate multiple antenna code design," IEEE Transactions on Information Theory, 47:2335-2367, Sept. 2001.

[67]. B. Lu and X. Wang, "Space time code design in OFDM systems," In Proceedings of the IEEE Global Telecommunications Conference, GLOBECOM, 2000, pp. 1000-1004.

[68]. B. Lu, Wang, and K. R. Narayanan, "LDPC based space time coded OFDM systems over correlated fading channels: performance analysis and receiver design," IEEE Transaction on Communications, 50(1):74-88, Jan. 2002.

[69]. Z. Liu, Y. Xin, and G. Giannakis, "Space time frequency coded OFDM over frequency selective fading channels," IEEE transaction on Signal Processing, 50:2465-2476, Oct. 2002.

[70]. A. F. Molish, M. Z. Win, and J. H. Winters, "Space time frequency (STF) coding for MIMO-OFDM systems," IEEE Communications Letters, 9:370-372, Sept. 2002. 
VITA

\section{YURI LABRADOR}

\section{EDUCATION}

$12 / 09$

Doctoral Candidate, Electrical Engineering

Florida International University

Miami, Florida.

$12 / 07$

Master of Science in Telecommunications and

Networking

Florida International University

Miami, Florida.

$06 / 96$

Bachelor of Science. Telecommunications

ISPJAE

Havana, Cuba.

\section{RECENT WORK EXPERIENCE}

Position: RF Engineer at Univision Networks Inc. Miami, Florida. (March 2000-present). Job Functions:

- Design and analysis of satellite communication systems.

- Link budget analysis

- Design of digital communication systems

- Analysis of error correction protocols

- Analysis of video compression algorithms

\section{PUBLICATIONS AND PRESENTATIONS}

1- Yuri Labrador, Masoumeh Karimi, Deng Pan, Jerry Miller, "Modulation and Error Correction in the Underwater Acoustic Communication Channel", International Journal of Computer Science and Network Security (IJCSNS), Vol. 9 No. 7 pp. 123-130, July, 2009.

2- Yuri Labrador, Masoumeh Karimi, Deng Pan, Jerry Miller. "An Approach to Cooperative Satellite Communications for 4G Mobile Systems", Journal of Communications (JCM), Accepted: August $15^{\text {th }} 2009$.

3- Yuri Labrador and Massoumeh Karimi, "OFDM MIMO Space Diversity in Terrestrial Channels", International Journal of Computer Science and Network Security (IJCSNS), Submitted: October $2^{\text {nd }} 2009$ 